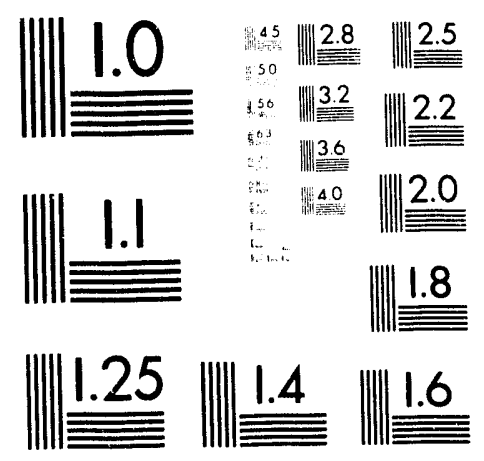



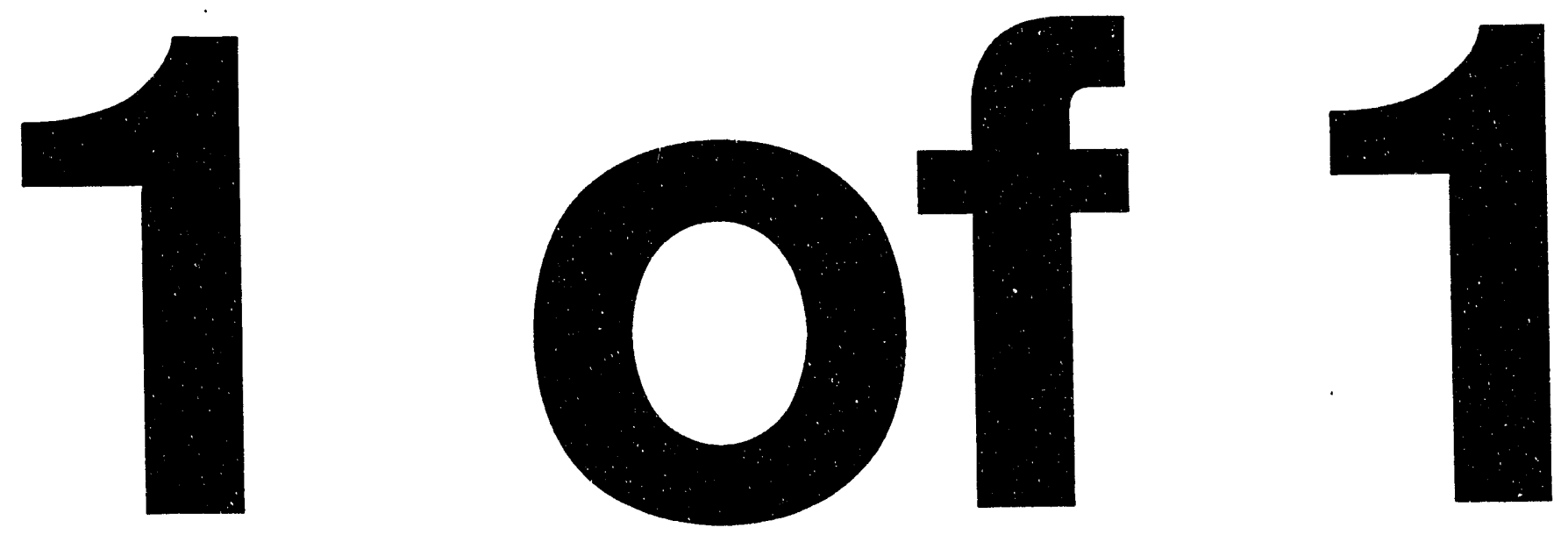
NUREG/CR-6081

$1 \mathrm{~S}$

\title{
Enhanced Removal of Radioactive Particles by Fluorocarbon Surfactant Solutions
}

Manuscript Completed: March 1993

Date Published: August 1993

\author{
Prepared by \\ R. Kaiser, O. K. Harling \\ G. E. Powers, NRC Project Manager
}

Entropic Systems, Inc.

P.O. Box 397

Winchester, MA 01890-0597

\section{Prepared for}

Division of Regulatory Applications

Office of Nuclear Regulatory Research

U.S. Nuclear Regulatory Commission

Washington, DC 20555-0001

NRC FIN L2575 
The Nuclear Regulatory Commission utilizes the Small Business Innovative Research (SBIR) program, to fund small scale research projects that may serve to enhance public health and safety and promote the development of small businesses. These projects are selected based upon the agency's determination that the research can eventually result in an independent and self sustaining business and application that will benefit the public safety and the NRC's prime directive to assure the same.

This report discusses the research results of the first phase of a two phase SBIR project on the use of perflurocarbons as a replacement for chlorofluorocarbons (CFCs) as a decontamination medium used in ultrasonic decontamination equipment. The research presented in this first report describes the radiation stability of several test flurocarbon surfactant solutions that could be used to replace the environmentally incompatable CFCs; the determination of decontamination factors using controlled test samples and radioactive contaminants; and finaliy the quantitative removal of radioactive contaminants from the cleaning solution to permit reuse of the cleaning medium. The report utilizes existing NRC guidance found in Regulatory Guide 8.23 as adapted from Regulatory Guide 1.86 as criteria for releasing decontaminated material. This is consistent with commission direction while the enhanced participatory rulemaking process to establish decommissioning criteria is underway.

NUREG/CR-6081 is not a substitute for NRC regulations, and compliance is not required. The approaches and/or methods described in this NUREG are provided for information only. Publication of this report does not necessarily constitute NRC approval or agreement with the information and recommendations contained herein.

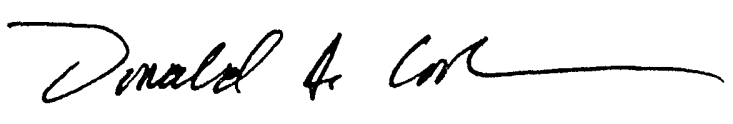

Donald A. Cool, Chief

Radiation Protection and Health

Effects Branch

Division of Regulatory Applications

office of Nuclear Regulatory Research 


\begin{abstract}
The proposed research addressed the application of ESI's particle removal process to the non-destructive decontamination of nuclear equipment. The cleaning medium used in this process is a solution of a high molecular weight fluorocarbon surfactant in an inert perfluorinated liquid which results in enhanced particle removal. The perfluorinated liquids of interest, which are recycled in the process, are nontoxic, nonflammable, and environmentally compatible, and do not present a hazard to the ozone layer.

The information obtained in the Phase I program indicates that the proposed ESI process is technically effective and economically attractive.

The fluorocarbon surfactant solutions used as working media in the ESI process survived exposure of up to $10 \mathrm{Mrad}$ doses of gamma rays, and are considered sufficiently radiation resistant for the proposed process. Ultrasonic cleaning in perfluorinated surfactant solutions was found to be an effective method of removing radioactive iron ( $\mathrm{Fe}(59))$ oxide particles from contaminated test pieces.

Radioactive particles suspended in the process liquids could be quantitatively removed by filtration through a $0.1 \mu \mathrm{m}$ membrane filter. Projected economics indicate a pre-tax pay back time of 1 month for a commercial scale system.
\end{abstract}




\section{TABLE OF CONTENTS}

Section

Page

Title page

Abstract

Table of Contents

List of Figures

List of Tables

Executive Summary

Acknowledgements

Abbreviations

1. INTRODUCTION

1.1 Identification and significance of the problem

1.2 Background and technical approach

1.2.1 Background: Particle adhesion mechanisms

1.2.2 Technical approach: particle removal with perfluorinated surfactant solutions

1.3 Research issues to be addressed

Program objectives

1.4 Anticipated benefits

2. RADIATION RESISTANCE OF PROCESS LIQUIDS 14

2.1 Introduction

2.2 Experimental methodology

2.2.1 Materials

2.2.2 Radiation exposure

2.2.3 Analytical methods $\quad 14$

2.3 Results

2.4 Discussion of results $\quad 15$

2.4.1 Stability of PF-5070 15

2.4.2 Stability of Krytox 157 FS 19

2.4.3 Stability of 1\% solution of Krytox 157 FS in PF-5070 19

2.5 Conclusions

3. DECONTAMINATION STUDIES 21

3.1 General methodology 21

3.2 Experimental procedure $\quad 21$

$\begin{array}{ll}\text { 3.2.1 Process materials } & 21\end{array}$

3.2.2 Experimental equipment and procedure $\quad 34$

$\begin{array}{ll}3.3 \text { Experimental results } & 35\end{array}$ 


\section{TABLE OF CONTENTS (CONTINUED)}

Section

Page

3.4 Discussion of results $\quad 45$

3.4.1 Effectiveness of decontamination solutions $\quad 45$

$\begin{array}{ll}3.4 .2 \text { Rate of decontamination } & 50\end{array}$

3.4.3 Effect of part structure $\quad 52$

3.4.4 Effect of particle size $\quad 55$

3.4.5 Sonication of electrical parts $\quad 55$

3.5 Conclusions $\quad 57$

4. FILTRATION OF PROCESS LIQUIDS

4.1 Introduction $\quad 58$

4.2 Experimental apparatus $\quad 58$

4.3 Experimental results $\quad 58$

4.4 Discussion of results $\quad 58$

4.5 Conclusions $\quad 61$

5. PROCESS DESCRIPTION AND ECONOMICS 62

5.1 Process Description $\quad 62$

5.2 Projected process economics $\quad 65$

5.2.1 Projected system capital cost $\quad 65$

5.2.2 Projected system operating cost $\quad 65$

$\begin{array}{ll}\text { 5.2.3 Economic Incentives } & 68\end{array}$

5.3 Conclusion $\quad 70$

6. FINDINGS, CONCLUSIONS AND RECOMMENDATIONS 71

$\begin{array}{ll}6.1 \text { Findings } & 71 \\ 61\end{array}$

6.1.1 Prociss Liquid Stability $\quad 71$

$\begin{array}{ll}\text { 6.1.2 Decontamination Studies } & 71\end{array}$

$\begin{array}{ll}\text { 6.1.3 Filtration Studies } & 71\end{array}$

6.1.4 Projected Process Economics $\quad 72$

6.2 Conclusions $\quad 72$

6.2.1 Process Liquid Stability $\quad 72$

6.2.2 Decontamination Studies $\quad 72$

$\begin{array}{ll}\text { 6.2.3 Filtration Studies } & 72\end{array}$

6.2.4 Projected Process Economics 72

$\begin{array}{ll}\text { 6.2.5 Overall Conclusions } & 72\end{array}$

$\begin{array}{ll}6.3 \text { Recommendations } & 73\end{array}$

7. REFERENCES $\quad 74$ 


\section{LIST OF FIGURES}

Figure No.

Page

1.1 Flow diagram for ESI cieaning process

1.2 Cleaning of silica contaminated glass slides in CFC-113 and perfluorinated liquids

1.3 Sequential ultrasonic cleaning of G-6 bellows in CFC-113 and FC-77/ surfactant solution

3.1 Size distribution of iron oxide powders used as contaminants in study

3.2 Suspended nut and bolt to be inserted in screw top metal can used as container for decontamination tests

3.3 Aluminum washer assemblies in decontaminations (left to right): single washer, washer sandwich with 7 mil gap, washer/screen sandwich

3.4 Elements of aluminum washer/screen/washer assembly 30

3.5 Printed circuit board "club sandwich" made from dual general-purpose PC board

3.6 Elements of printed circuit board "club sandwich"

3.7 Cumulative removal of particles $>2 \mu \mathrm{m}$ by sonication in different fluorinated liquids

5.1 Decontamination process flow diagram 


\section{LIST OF TABLES}

Table No.

Page

2.1 Effect of gamma radiation on selected properties of perfluoroheptane (PF-5070)

2.2 Effect of gamma radiation on selected properties of a 1 wt-\% solution of Krytox 157FS(M) in perfluoroheptane (PF-5070)

2.3 Effect of gamma radiation on selected properties of Krytox 157FS(M) surfactant

3.1 Neutron irradiation for analysis of iron $(+3)$ oxide powders used in cleaning experiments

3.2 Composition of iron $(+3)$ oxide powders used in cleaning experiments as measured by instrumental neutron activation analysis

3.3 Neutron irradiation of iron $(+3)$ oxide powders used in cleaning experiments

3.4 Configuration of radiation detection equipnent used in study

3.5 Summary results of decontamination experiments with an aluminum washer and aluminum washer sandwiches

3.6 Percent decontamination achieved in experiments with an aluminum washer and aluminum washer sandwiches

3.7 Summary results of decontamination experiments with aluminum washer screen sandwiches

3.8 Percent decontamination achieved in experiments with aluminum washer screen sandwiches

3.9 Summary results of decontamination experiments with nuts and bolts and with test can

3.10 Percen decontamination achieved in experiments with nuts and bolts and with test can

3.11 Summary results of decontamination experiments with a printed circuit board "club sandwich"

3.12 Results summary of decontamination studies 44

3.13 Maximum allowable radiation limits for surface 46 contamination 


\section{LIST OF TABLES (CONTINUED)}

Table No.

3.14 Decontamination levels attained with parts examined in study

3.15 Number of test samples successfully decontaminated as a funtion of decontamination criterion

3.16 Post-cleaning examination of components of PC board test assembly

3.17 Effect of sonication in perfluorinated liquids on the performance of electrical resistors

4.1 Post cleaning filtration of contaminated CFC-113

4.2 Post cleaning filtration of contaminated

PF-5070/ surfactant solution

60

5.1 Estimated Cost of purchased equipment and process materials for a prototype decontamination system

66

5.2 Estimated projected operating costs of a small ( 5 gallon bath) ESI decontamination system

5.3 E:timated projected operating costs of a commercial scale (200 gallon bath) ESI decontamination system 


\section{EXECUTIVE SUMMARY}

\section{Identification and Significance of the Problem}

In the nuclear industry, facilities and their components inevitably become contaminated with radioactive materials. While contamination may be due to a chemical reaction between radioactive process materials with the materials of construction of these facilities and components, it is often due to the deposition of thin films of fine adherent radioactive particles that are not chemically bonded to the substrates. This is especially true in the case of accidental discharges.

Design and operational procedures contribute to minimize these effects, but decontamination is a necessary process in reducing radiation levels in the working environment. Whereas facilities are occasionally cleaned either for reuse or decommissioning, components from active areas commonly require decontamination for reuse or maintenance operations on a much more frequent schedule.

Decontamination processes are similar to traditional surface cleaning processes which involve the removal of unwanted nonradioactive surface contaminants, except as to the types of contaminants and, to a certain extent, the degree of removal. Nuclear decontamination requires an essentially complete level of contaminant removal, a level of cleaning perfection which is required by only select manufacturing operations which are very sensitive to foreign contaminants, such as the semiconductor industry.

Chlorofluorocarbons, such as trichlorotrifluoroethane (CFC-113), have been of particular interest to the nuclear industry for they offer the ability to clean electrical components without damage, and can clean product contaminated material without the risk of criticality (Bond and Keasey, 1984). In particular, high pressure spraying of CFC-113 has been found to be an effective means of removing radioactive particulate contamination from devices, including electrical equipment and hardware (Ashley, 1984).

Decontamination processes based on CFC's are now obsolescent. Because of new evidence that the earth's atmospheric ozone shield may be weakening more than previously thought, President Bush announced in early 1992 that, under the provisions of the Clean Air Act of 1990, the production of all chemicals known to be sources of atmospheric chlorine, including CFC-113, will be banned after December 31, 1995. As a consequence, after that date, no viable method of reclaiming radioactively contaminated electronic and similar sensitive high value equipment will be available to the nuclear industry. 


\section{Technical Approach}

This report discusses the application of a novel particle removal process developed by Entropic Svstems, Inc. (ESI) to the decontamination of critical parts and instruments that are contaminated by the presence of small radioactive particles that adhere to the surfaces of this equipment.

ESI has developed a novel, environmentally compatible process to remove small particles from solid surfaces which is more effective than spraying or sonicating with CFC-113. The process uses inert perfluorinated liquids as the working media; the liquids have zero ozone depletion potential, are nontoxic, nonflammable, and are generally recognized as nonhazardous materials. In the ESI process, the parts to be cleaned are first sprayed or sonicated with a dilute solution of a high molecular weight fluorocarbon surfactant in an inert perfluorinated liquid to effect particle removal, the parts are then rinsed with the perfluorinated liquid to remove the fluorocarbon surfactant applied in the first step, the residual rinse liquid is evaporated from the parts into an air or nitrogen stream from which it is subsequently recovered.

Phasex Corporation of Lawrence, MA, and ESI recently completed Phase I of an Air Force sponsored SBIR program (AF 91-186) to develop new processes for precision parts cleaning to replace existing CFC-113 cleaning operations. The approach taken on this contract was to remove oils by supercritical fluid (Phasex) and to remove particles with fluorocarbon surfactant solutions (Gallagher, Krukonis and Kaiser, 1992). Phasex and ESI were awarded a Phase II program which started in June 1992.

An order of magnitude increase in the rate and extent of particle removal was observed when inertial guidance instrument parts were ultrasonically cleaned in a dilute solution of HFPO carboxylic acid in a perfluorinated liquid instead of CFC-113. It has also been demonstrated that adsorbed HFPO carboxylic acid can be removed from metal washers and from instrument parts by simple extraction with a surfactant free fluorinated liquid.

Nuclear contamination is inherently a surface phenomenon. Much nuclear waste is the result of the deposition of radioactive particles onto nonradioactive substrates. The presence of radioactive particles is responsible for all "smearable" contamination, and, if the radioactive particles are small enough, for some of the fixed contamination. Since radioactivity does not influence the physical chemistry of particle adhesion, the ESI process should be just as effective in removing radioactive particles as nonradioactive particles.

The concept would be to use the ESI process to remove radioactive particles from large, and therefore costly to dispose of, contaminated objects of high inherent value. The suspended radioactive particles would then be removed from the process liquid by filtration, so that the liquid could then be reused. The radioactive 
particles from the decontaminated parts accumulate on the filter. The filter and the captured particles are periodically disposed of as radioactive waste. The perceived advantages of the proposed process are:

a. a great reduction in the volume of the radioactive waste that needs to be disposed of, and

b. the ability to salvage valuable equipment.

\section{Research Issues to be Addressed - Program Objectives}

A key issue that needed to be addressed was determining how effective the ESI process was in terms of reducing residual radioactivity of complex parts. The measurement of residual radioactivity allows one to measure the residual number of particles that remain on the inner surfaces of a complex part, which can not be examined by line of sight, after cleaning. The prior experiments with devices of complex shape measured the number of particles in the cleaning liquid before and after contacting the device being cleaned. These measurements allowed one to compare the cleaning efficiency of different media, but did not provide any data on residual particles left on the device after cleaning. One of the advantages of performing cleaning experiments with radioactive particles is that measurement of the radioactivity of a part before and after a cleaning step provides a direct measure of both the number of particles that were removed as well as the number of particles that remained on the surfaces of this part.

The primary program objective was to demonstrate that radioactive particles could be quantitatively removed from complex parts by perfluorinated surfactant solutions, so as to decontaminate these parts. A corollary objective was to demonstrate that the rate and extent of particle removal, under comparable conditions of shear, is significantly greater in these solutions than in CFC-113 or surfactant free fluorocarbon liquids.

Another important goal was to demonstrate that radioactive particles suspended in the cleaning liquid could be quantitatively removed by simple filtration from this liquid in such a way that the radioactive particles could be simply disposed of and that the cleaning liquid could be recycled for further use.

A major uncertainty with regards to fluid consumption is the effect of high energy radiation on the physical and chemical properties of the fluorinated surfactant solutions. While data were available on the radiation stability of Krytox 143, the composition of which is similar to that of the fluorocarbon tail of the fluorinated surfactant, Krytox 157FS, no data were available on the radiation stability of Krytox 157FS, or of solutions of this material in perfluorinated liquids. The 
presence of a carboxylic acid end group could render this HFPO oligomer more susceptible to radiation than the analogous fluorine endcapped oligomer.

Determining the radiation stability of the process fluids, so as to establish that the fluorinated surfactant solutions used as the working media in the ESI particle removal / decontamination process exhibit sufficient radiation stability to allow them to be used effectively and economically under projected conditions of use, was therefore another major technical objective of the Phase I program.

The final goal of the program was to develop, based on the experimental data to be obtained in Phase I, as well as other ESI data, a design for a decontamination process that would be the basis of a prototype decontamination system that could be built, assembled and tested by ESI if it were awarded a Phase II award.

\section{Finciings}

\section{Stability of Process Liquids}

The radiation stability of perfluoroheptane (3M's PF-5070), poly(hexafluoropropylene oxide) carboxylic acid (Krytox 157FS-M made by DuPont), and of a 1 wt $\%$ solution of Krytox 157FS-M was determined by exposing samples of these materials to gamma rays in the spent fuel pool of the MIT Nuclear Reactor (MITR2). The maximum dose rate in the pool is $3 \times 105 \mathrm{R} / \mathrm{hr}$. Samples received doses ranging from approximately $0.5 \mathrm{Mrad}$ to $10 \mathrm{Mrad}$.

While soine changes in the structure of the process liquids occured, there was no catastrophic damage even at the highest radiation levels used (10 Mrad), and which is significantly higher than the radiation levels the process liquids would be exposed to in decontamination operations.

Radiation stability is not a barrier to the use of perfluorinated liquids as decontamination media.

\section{Decontamination Studies}

Well characterized radioactive iron oxide (Fe2O3) powders were used as model contaminants in the Phase I decontamination tests. The reasons for choosing this material include:

a. The $\mathrm{Fe}(59)$ isotope, created by thermal neutron activation, has a half-life of 44.5 days. This half life is of the same duration as the elapsed time of the experimental program that was performed. However, it is not so long as to create significant and costly post-experimental disposal problems. The radiation fror.. Fe(59) is conveniently measurable.

b. Pigment grade iron oxides of high purity are available commercially in a range of particle sizes of interest. 
c. Radioactive iron oxide (rust) is not an uncommon contaminant in operational facilities.

A well characterized grade of iron oxide powder that had been air classified into two fractions was obtained from the Donaldson Company of Minneapolis, MN. The particle size distribution of the feed material ranged from less than $1 \mu \mathrm{m}$ to 40 $\mu \mathrm{m}$, with a weight average particle size of $11.9 \mu \mathrm{m}$. The particle size distribution of the fine fraction ranged from about $0.5 \mu \mathrm{m}$ to $2.4 \mu \mathrm{m}$, with a weight average particle size of $1.3 \mu \mathrm{m}$. The particle size distribution of the coarse fraction ranged from about $2.4 \mu \mathrm{m}$ to $40 \mu \mathrm{m}$, with a weight average particle size of $12.5 \mu \mathrm{m}$.

The composition of these iron oxide powders was determined by Instrumental Neutron Activation Analysis (INAA) at the MIT Nuclear Reactor Laboratory (MITNRL). These powders did not contain any significant amount of elements that could form hazardous or long lived isotopes.

Decontamination tests were performed with both the feed material and the fine fraction as the model contaminant after these had been irradiated for approximately one day in the MTTR2.

Three cleaning liquids were used in the study: CFC-113 as a baseline, perfluoroheptane (3M's PF-5070), and a $1 \mathrm{wt}$ \% solution of Poly (hexafluoropropylene oxide) carboxylic acid [DuPont's Krytox 157FS(M), Lot 14] in PF-5070.

Decontamination tests were performed on flat coupons, nuts and bolts, test assemblies of washers bolted together, and on a test container that had been contaminated as a result of its use in a prior test. These test pieces were small, one inch $(2.5 \mathrm{~cm})$ on a side or smaller.

The amount of radioactive iron oxide present on a test sample was measured by counting the gamma radiation emitted when the test sample of reproducible geometry was placed near a scintillation counter with a 3 in $\times 3$ in $(7.5 \mathrm{~cm} \times 7.5 \mathrm{~cm})$ sodium iodide crystal scintillation detector. The detector was contained in a shielded chamber that was large enough to accommodate the $4 \mathrm{oz}$ vessels in which the experimental work was being performed. It was therefore possible to measure the radiation characteristics of either the test sample supported in an unused, clean vessel, or the decontamination liquid in its test vessel.

Test pieces were contaminated by immersion in a suspension of radioactive iron oxide particles in isopropanol. After evaporating the alcohol, the radioactivity of the contaminated test piece was measured. The test piece was decontaminated by placing it in a clean container containing a given volume of cleaning liquid free of contaminating particles. The container was placed in an ultrasonic bath containing a coupling liquid (e.g. water), and sonicated for a short period of time $(5 \mathrm{~min})$. The supported test piece was then removed from the beaker, and its 
radioactivity was measured. The radioactivity of the test piece, and of the cleaning liquid in the container; before inserting the test piece in the cleaning solution and after its removal; are a quantitative measure of the amount of decontamination (i.e. particle transfer from the test piece to the liquid, that occurred during the test).

The test piece was then placed in a new container containing fresh cleaning liquid, and again subjected to the test procedure described above. In prior work on the removal of nonradioactive particles it was generally observed that, with each sequential process step, additional particles are removed from the test piece, but that the number of particles removed and their size both tend to decrease. Test sequences were carried out until no further significant transfer of radioactive particles from the test piece to the cleaning liquid occurred.

The level of radioactivity of fifteen parts contaminated with micron sized, radioactive iron ( $\mathrm{Fe}(59))$ oxide powder was decreased by one to better than two orders of magnitude by ultrasonic cleaning in CFC-113 or perfluorinated liquids to the values indicated in the following table:

\begin{tabular}{|c|c|c|l|}
\hline $\begin{array}{c}\text { Number of } \\
\text { Samples }\end{array}$ & $\begin{array}{c}\text { Residual } \\
\text { Radioactivity } \\
\mathrm{Bq}\end{array}$ & $\begin{array}{c}\text { Residual } \\
\text { Contamination } \\
\text { decays/min./100 } \mathrm{cm}^{\wedge} 2\end{array}$ & \multicolumn{1}{|c|}{ Type of Contamination } \\
\hline 15 & $<38$ & $<11,000$ & Fixed, Low risk B or x-ray \\
13 & $<9$ & $<2,200$ & Removable, Low risk B or x-ray \\
12 & $<3$ & $<1,100$ & Fixed, B or x-ray \\
5 & $<1$ & $<220$ & Removable, B or x-ray \\
\hline
\end{tabular}

The residual contamination values cited above are the radiation limits listed in NRC Regulatory Guide 8.23 for allowable radiation limits in unrestricted areas for fixed and removable contaminants containing either low risk beta or x-ray emitters, such as the iron oxide powders used in the study, or unspecified beta or x-ray emitters.

Perfluorinated surfactant solutions are at least as effective as perfluorinated liquids and CFC-113 as decontamination media, and may be more effective. Decontamination occurs rapidly, with most of the radioactive particles being removed with the first minutes of sonification. The differences in decontamination levels achieved is mainly a function of parts complexity.

Sonication of a part contaminated with radioactive particles in a fluorinated liquid is an effective method of decontamination.

\section{Filtration Studies}

Filtering the contaminated process liquids from the parts decontamination operation 
through a $0.1 \mu \mathrm{m}$ rated mernbrane microfilter was found to quantitatively remove the radioactive iron oxide particles from suspension. Within the statistics of the measurements, the filtrates were found not to be radioactive.

The process liquids can be simply decontaminated by filtration, and can thus be recycled for further use. The filter also provides a simple, compact support for the disposal of the radioactive particles removed from the parts being decontaminated.

\section{Projected Process Economics}

It is projected that the capital cost of a pilot system capable of processing 0.2 $\mathrm{ft}^{\wedge} 3$ per hour of radioactive waste would be approximately $\$ 100,000$. Operating costs on a one shift basis are estimated to be about $\$ 50$ / hour.

A commercial scale unit capable of processing $10 \mathrm{ft}^{\wedge} 3$ of radioactive waste per hour would be approximately $\$ 400,000$. Operating costs on a one shift basis are estimated to be $\$ 125 / \mathrm{hr}$.

Assuming credits, conservatively estimated to be only $\$ 300 / \mathrm{ft}^{\wedge} 3\left(\$ 100 / \mathrm{ft}^{\wedge} 3\right.$ for reduced waste disposal costs and $\$ 200 / \mathrm{ft}^{\wedge} 3$ for the value of the recovered equipment), the commercial scale system would be financially attractive with a pretax pay back time of 1 month. For the pilot system, if operated continuously, the pay back time would be 9 months, which is still very favorable.

\section{Conclusions and Recommendations}

The information obtained in the Phase I program indicates that the proposed ESI decontamination process is technically effective and economically attractive.

It is recommended that the ESI decontamination process be further developed in a Phase II program.

\section{Anticipated Benefits}

Successful implementation of the ESI process to nuclear decontamination of immense value to the nuclear industry and the U.S. government, and would definitely be of commercial value. The industry would be provided with a method and a system to remove undesirable radioactive particles from electronic and electrical equipment without damaging this equipment, and thus allowing it to be reused or disposed of as nonradioactive waste. Disposal costs have been increasing at an astronomic rate and will be several times higher than the current rate within the next decade. 
In more general terms, the proposed research would be more than an environmentally suitable alternate for high pressure CFC-113 decontamination systems, since it would result in higher particle removal efficiencies. If the proposed process is as successful as anticipated, equipment which would other'vise be buried in a nuclear waste site would be available for reuse, with a resulting dual cost reduction: lower waste disposal costs, and lower capital replacement costs. 


\section{ACKNOWLEDGEMENTS}

This study was performed under the auspices of the Small Business Innovation Research Program of the Nuclear Regulatory Agency. It provided Entropic Systems, Inc. (ESI) with the support needed to demonstrate the applicability of its particle removal technology to an area of growing 1 Jational importance.

The help and cooperation of Mr. G. Edward Powers, the NRC Project Officer, is acknowledged.

The study could not have been performed without the support and assistance of Dr. Otto K. Harling, Consultant to ESI and Director of the Nuclear Reactor Laboratory at the Massachusetts Institute of Technology, Cambridge, MA.; and to the staff of the MITNRL.

The assistance of Dr. Ihan Olmez is particularly noted. He allowed ESI access to his radiochemistry laboratory, which allowed the study to be performed. He also prepared and analyzed the radioactive iron oxide powders that were used as model contaminants in the study.

The assistance of Mr. R. R. Ambati, ESI Research Engineer, is also acknowledged. Mr. Ambati performed the evaluation of the fluorinated liquid samples subjected to gamma ray exposure.

Robert Kaiser

Principal Investigator 


\section{ABBREVIATIONS}

ESI

INAA

k

MITNRL

MITR2
Entropic Systems, Inc.

Instrumental Neutron Activation Analysis

Boltzman's Constant

MIT Nuclear Reactor Laboratory

MIT Nuclear Reactor 


\section{INTRODUCTION}

\subsection{Identification and Significance of the Problem}

In the nuclear industry, facilities and their components inevitably become contaminated with radioactive materials. While contamination may be due to a chemical reaction between radioactive process materials with the materials of construction of these facilities and components, contamination is often due to the deposition of thin films of fine adherent radioactive particles that are not chemically bonded to the substrates, especially in the case of accidental discharge.

Design and operational procedures contribute to minimize these effects, but decontamination is a necessary process in reducing radiation levels in the working environment. Whereas facilities are occasionally cleaned either for reuse or decommissioning, components from active areas commonly require decontamination for reuse or maintenance operations on a much more frequent schedule.

Decontamination has been defined as the removal of unwanted radionuclides from surfaces (Ayres, 1970). Decontamination processes are similar to traditional surface cleaning processes which involve the removal of unwanted nonradioactive surface contaminants, except as to the types of contaminants and, to a certain extent, the degree of removal. Nuclear decontamination requires an essentially complete level of contaminant removal, a level of cleaning perfection which is required by only select manufacturing operations which are very sensitive to foreign contaminants, such as the semiconductor industry. As a result, most of the decontamination processes now used in the nuclear industry involve abrasive mechanical operations or aggressive cleaning solutions (Ayres, 1970; Rankin and McGlynn, 1989). The mechanical methods are cestructive in that, in order to attain the required degree of decontamination, the external surface layer of the object being decontaminated as well as the contaminating radionuclides. Most cleaning solutions are water base, and may contain detergents, sequestering agents, acids or bases, inhibitors, etc. These solutions are usually applied under conditions of high shear, with apparatus which can range from agitated tanks to high pressure jets.

With the exception of chlorinated solvents, such as trichloroethylene (TRIC), and chlorofluorocarbons, such as trichlorotrifluoroethane (CFC-113), organic liquids have been used to a very limited extent as cleaning media in the nuclear industry because of safety and flammability concerns. CFC-113 base liquids have been of particular interest to the nuclear industry for they offer the ability to clean electrical components without damage, and can clean product contaminated material without the risk of criticality (Bond and Keasey, 1984). In particular, high pressure spraying of CFC-113 has been found to be an effective means of removing radioactive particulate contamination from devices, including electrical equipment and hardware (Ashley, 1984). 
Decontamination processes based on CFC's are now obsolescent. Because of new evidence that the earth's atmospheric ozone shield may be weakening more than previously thought, President Bush announced in early 1992 that, under the provisions of the Clean Air Act of 1990, the production of all chemicals known to be sources of atmospheric chlorine, including CFC-113 and TRIC, will be banned after December 31, 1995. As a consequence, after that date, no viable method of reclaiming radioactively contaminated electronic and similar sensitive high value equipment will be available to the nuclear industry.

\subsection{Background and Technical Approach}

This report discusses the application of a novel particle removal process developed by ESI to the decontamination of critical parts and instruments that are contaminated by the presence of small radioactive particles that adhere to the surfaces of this equipment.

In this section of the report, mechanisms of particle adhesion are first discussed. This is followed by a discussion of ESI's approach to the removal of such particles, supported by the presentation of data obtained on the removal of nonradioactive particles from test parts. The case for using this technology for nuclear decontamination is then made.

\subsubsection{Background: Particle Adhesion Mechanisms}

Very small solid particles are generally observed to adhere tenaciously and nonspecifically to other solid surfaces, and can not be removed by simple mechanical means. There is a large body of physical evidence that suggests that their adhesion is due to relatively weak secondary valence forces, or van der Waals' forces, that act between adjacent solid bodies, and whose magnitude becomes much larger than that of other forces that can be applied to a solid as its characteristic size decreases to the order of $1 \mu \mathrm{m}\left(10^{\wedge}-4 \mathrm{~cm}\right)$ or less. Adhesion of fine particles to solid surfaces in general is believed to be due mainly to secondary valence interaction between the particles and these surfaces, rather than by primary valence bonding, electrostatic charges, or surface tension forces due to capillary condensation of a liquid.

General explanations for the nature of Van der Waals forces were first proposed by London (1930), and more recently by Lifschitz (1954). These explanations are both based on quantum theory which considers that all atoms, even in their ground state, possess rapidly fluctuating dipole moments which lead to an attraction between them. As a result of this interaction, significant attractive forces exist between any two adjacent solid objects. In the case of a small sphere (i.e. a particle) and a flat plane (i.e. a substrate), these attractive forces increase as a function of particle diameter and as an inverse power function of the distance separating the surface of the particle from the surface of the substrate (Tabor, 1969). With decreasing particle diameter, it becomes progressively more difficult to remove a solid particle 
from a solid surface by inertial means because the value of the ratio of the force of attraction to the weight of a particle, FS/W, increases rapidly as the diameter of the particle decreases.

The effective surface-tc-surface separation between a particle deposited from the atmosphere and a solid surface is mainly determined by the surface roughness of the particle and of the surface, and by the thickness of gas films adsorbed on these surfaces. Experimental values of the surface separation range from $3 \AA$ to more than $1,000 \AA$ depending on the surface roughness of the objects (Zimon, 1969). For particles of sub-micron size, the distance of separation would be expected to range from $3 \AA$ to less than $100 \AA$ (which corresponds to $1 \%$ of the diameter of a $1 \mu \mathrm{m}$ particle). For particles of the order of $1 \mu \mathrm{m}$ in diameter, secondary valence forces are one to four orders of magnitude greater than the weight of a particle. In general, such particles would be expected to adhere to a substrate, but, under favorable circumstances, such particles might be removed by the application of inertial forces with an acceleration of many g's. For particles of the order of 0.1 $\mu \mathrm{m}$ in diameter, secondary valence forces are more than five orders of magnitude greater than the weight of a particle. Such particles can not be removed from a substrate by inertial forces that can be practically applied.

Whereas it is extremely difficult to modify the interaction between the surface of a fine particle and that of an adjacent solid in the gas phase, this can be readily accomplished if the solids are immersed in a liquid phase with certain characteristics. As in the gas phase, solid particles immersed in a liquid phase are also subject to secondary valence forces of attraction which can result in their adherence to other solid surfaces. However, in the liquid phase, it is possible to induce the generation of repulsive forces between the adjacent surfaces that are larger than the secondary valence forces responsible for particle adhesion, as evidenced by the existence of stable colloidal dispersions of particles that are significantly smaller than $1 \mu \mathrm{m}$ in diameter.

The stability of colloidal dispersions in a liquid phase is due to the development of repulsive forces between particles of greater magnitude than the attractive forces discussed above, and to the thermal energy, $\mathrm{kT}$, of the suspended particles, as evidenced by Brownian motion. The stability of hydrosols (aqueous colloids) is usually attributed to the formation of an electrostatic double layer around a particle resulting from the adsorption of charged species on or around the particle surface (Verwey \& Overbeek, 1951). Stabilization occurs because of the electrostatic repulsion between two similarly charged particles.

A second more general mechanism of stabilization that applies to both aqueous and nonaqueous colloidal dispersions results from the entropic repulsion of large, solvated molecules that are adsorbed on the particle surfaces. These adsorbed molecules form a thick film of essentially bound liquid. When two particles collide, they are separated by a minimum distance equal to twice the film thickness. If the film thickness is sufficiently large, the secondary valence interaction between 
particles becomes negligible in comparison to their thermal energy, $\mathrm{kT}$, and which, under these circumstances, becomes the dominant factor. It has been estimated that the condition necessary for the formation of a stable dispersion is that the ratio of film thickness to particle diameter be $\geq 0.15$ (Mackor, 1951). This mechanism of entropic repulsion predominates in the formation of colloidal suspensions in nonaqueous media, in which sub-micron particles are stably suspended in a liquid due to the presence of polar molecules that can both adsorb on the surface of the solid particles and be solvated by the liquid medium.

\subsubsection{Technical Approach: Particle Removal with Perfluorinated Surfactant Solutions}

\subsubsection{General Concept}

The fact that fine particles can be stably dispersed in a surfactant solution (as compared to their adherent properties in the gas phase) forms the basis of Entropic Systems Inc.'s (ESI) process for removing undesirable foreign particles from a solid surface. A key component of this approach is the use of solutions of high molecular weight surfactants in an inert carrier liquid to effect particle removal. The unique characteristics of these high molecular weight surfactants are that:

a) they can result in a relatively thick solvated shell around the particles, which promotes the detachment of solid particles from a solid substrate, and

b) they are reversibly adsorbed on the solid surfaces being treated, and that adsorption is controlled by varying their concentration in the liquid phase.

The proposed decontamination process is described below and is outlined in Figure 1.1. A piece of equipment whose surfaces are contaminated with foreign radioactive particles, is immersed in a washing solution which does not dissolve nor react irreversibly with any of the materials of construction of this piece of equipment. The washing solution contains a surfactant which adsorbs on the surfaces of the equipment and of any foreign particles present, forming solvated films on these surfaces. These solvated films reduce the interaction between the particles and the underlying surfaces. Because of thermal energy, the solvated particles are detached from the underlying surface. Mechanical agitation, as provided, for example, by an ultrasonic bath, or flow of sprayed liquid past the surfaces of the piece of equipment, promotes the dispersion of these detached particles into the bulk fluid.

Once suspended, these solvated particles will not reattach to the underlying surfaces, as could occur if the solvated films were not present. The washing liquid which contains the foreign particles in suspension is then physically separated from the piece of equipment.

The suspended radioactive particles are then separated from the washing liquid. This can be accomplished by a standard solid-liquid separation process, such as filtration or centrifugation. The separated radioactive particles are then removed from the 

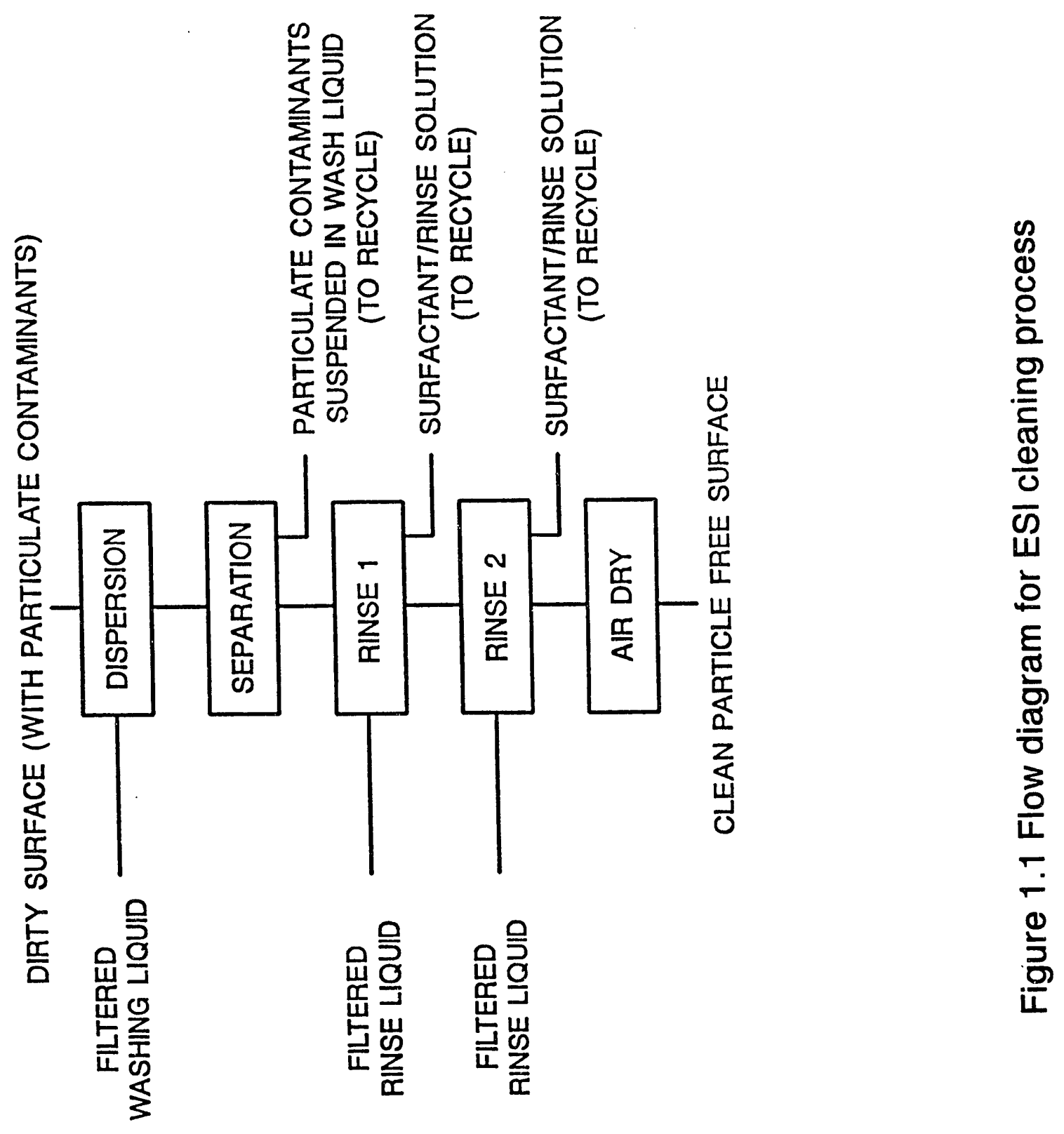
process for disposal. If filtration is used, one can either periodically dispose of the particle laden filter, or use a back-flushable filter. The preferred separation process will depend on the specifics of the operation. For small scale operations, the simplest process would entail filtration and the subsequent disposal of the contaminated filters, which would then be replaced by fresh filters.

The work piece is then rinsed with a liquid that has an affinity for the surfactant used in the previous step to effect the quantitative removal of any surfactant that is adsorbed on its surfaces. This rinsing liquid should not interact with any of the materials used in the construction of the piece of equipment being decontaminated. Preferably, this rinsing liquid should have the same composition as the carrier liquid used to prepare the surfactant solution used in the first step. The rinsing process is continued, by the addition of fresh rinse liquid, until there are no detectable traces of surfactant left on the surfaces of the equipment being treated.

Any residual rinsing liquid still adhering to the equipment can be removed mechanically (i.e. by centrifugation) and/or by evaporation into a flowing gas stream from which it is subsequently recovered by standard means, such condensation, adsorption, or pervaporation.

The product of the process is a decontaminated piece of equipment from which foreign, radioactive particles, adhering to its surfaces because of secondary valence forces, have been removed, but is otherwise unchanged.

As also indicated in Figure 1.1, all the fluid streams that come into contact with the equipment being decontaminated are filtered before the decontamination chamber to remove any foreign particles suspended in the process liquids, and which could interfere with the process. These process filters should be capable of removing all particles of a size large enough to be of concern.

While the various liquids used in the above process could be discarded after one pass, for economic reasons, it will be desirable to be able to recover and recycle them. The used washing liquid is recovered after removing the suspended radioactive particles. Makeup surfactant and carrier liquid are added to maintain a washing liquid of reasonably constant composition.

By using a volatile rinse liquid and a nonvolatile surfactant, used rinsing liquid, which contains traces of surfactant, is most easily recovered by distillation. The overhead product is purified rinse liquid that is returned to the process. The still bottoms are a solution of surfactant in rinse liquid that could be recycled as makeup washing solution.

\subsubsection{Preferred Cleaning Agents}

The choice of cleaning agents that may be used is constrained by the very strict requirements that the cleaning/decontamination method must meet if it is to be successfully implemented. These include: 
a) The removal of very small particles without leaving behind any residues, and in a manner that does not alter the physical and chemical properties of the equipment being decontaminated.

b) Compatibility with existing established industry operating practices.

c) No new risks or hazards are introduced into the work place in terms of flammability, toxicity or environmental compatibility.

All the above requirements can be satisfied by a select group of perfluorinated liquids that would be used as the wash solution carrier liquid, in conjunction with high molecular weight perfluorinated surfactants, and as the rinse solution. These fluorinated liquids are obtained by replacing carbon bound hydrogen atoms with fluorine atoms in a variety of organic compounds. These perfluorinated iiquids, as a class, exhibit a remarkable degree of inertness and resistance to chemical attack. They are nonpolar and have essentially no solvent action. They principally have an affinity for other highly fluorinated fluids. They are nonfammable and have a low order of toxicity. Fluorocarbons appear to be at least as stable toward ionizing radiation as some aromatic hydrocarbons. According to Heine (1962), perfluorinated liquids can withstand up to $10^{\wedge} 8$ rads of either gau. una or electron radiation before any significant change in their physical or chemical properties occur. Because of these combinations of properties, perfluorinated liquids have been used as test media for electronic components and devices, and have been specified as the test media of choice in a number of military standards, such as MIL-STD 833, MIL-STD 750, and MIL-STD 202. It is to be noted that tests involve the physical immersion of the electronic equipment in the perfluorinated liquids.

The fluorinated liquids of interest do not contain chlorine, and, in contrast to chlorofluorocarbons (CFC's), they are environmentally stable and do not present a hazard to the ozone layer (Stolarsky and Rundel, 1975). Because of their stability, perfluorinated liquids are not broken down in the atmosphere for a long period of time, and thus exhibit a potential for global warming. However, because of their cost, in any process applications, losses of these liquids have to be minimal for economic reasons. The likely concentration of these materials in the upper atmosphere will remain miniscule (in comparison to methane and carbon dioxide, and they are thus not considered to be a major threat to global warming (Ko et al, 1991).

Preferred fluorinated liquids for the proposed process have a boiling puint at ambient pressure in the range of from $35^{\circ} \mathrm{C}$ to $150^{\circ} \mathrm{C}$ to facilitate removal of carrier liquid at temperatures that do not harm the object being cleaned. $3 \mathrm{M}$ Corporation's PF-5070 Performance Fluid, a perfluoroalkane that is predoninantly perfluoroheptane, and 3M's FC-77 Fluorinert Electronic Liquid, a mixture of perfluorooctane and a cyclic perfluorooctylether, are examples of such fluids.

By themselves, perfluorinated liquids are not good dispersing media. However, as 
outlined in U.S. Patent 3,784,471 (Kaiser, 1974), stable dispersions of sub-micron particles of widely varying composition can be forn ed in a mixture of a perfluorinated liquid and a high molecular weight ?erfluorinated surfactant which contains preferably 20 to 100 carbon atoms and one or more polar groups capable of interacting with a solid surface. These polar groups include species with an active hydrogen atom, such as carboxylic acids and alcohols. The surfactant preferably has a much higher boiling point than the carrier liquid with which it is used to facilitate the recovery of used process solutions. It is also important that the surfactant be easily removed trom the surface to be cleaned, as by rinsing with surfactant free fluorocarbon liquid; otherwise, the cleaning process will merely result in the substitution of one contaminant for another. Surractants with a high molecular nonpolar moiety are more readily desorbed than otherwise comparable low'er molecular surfactants, and thus are preferred.

Surfactants which are suitable for the proposed process include polyhexafluoropropylene oxide (HFPO) carboxylic acids which can be represented by the following formula:

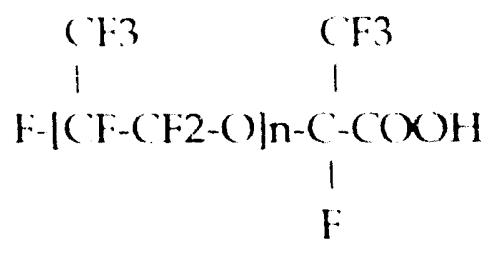

where $\mathrm{n}$ is an integer from 1 to 50 . Preferred surfactants are HFPO carboxylic acids with average molecular weights of from about $2,000(n=11)$ to $8,500(n=50)$ Dalton, marketed under the Krytox 157FS trade designation by E.I. Du Pont de Nemours \& Co., Inc. These materials are nonvolatile (vapor pressure of less than $10^{\wedge}-6$ torr), and are fully miscible with perfluorinated liquids as well as with CFC-113.

According to Du Pont (1983), Krytox 143 oils (which are fluorine endcapped HFPO polymers and do not have a carboxylic acid terminal group) are quite stable to radiation when compared with many materials used as lubricants or power fluids. In general, irradiation of Krytox 143 causes minor depolymerization with a consequent decrease in viscosity and the formation of volatile products, but no insoluble solids or sludge. In one test, exposure of a sample of Krytox $143 \mathrm{AC}$ to $10^{\wedge} 8$ rads of electron bombardment at room temperature resulted in a $21 \%$ ' crease in viscosity. There was no sludge formation or any visual change in the appe:sance of the sample.

\subsubsection{Particle Removal with Perfluorinated Surfactant Solutions}

The ability to stably suspend a wide variety of sub-micron solid particles, including uraniun dioxide, in dilute solutions of perfluorinated HFPO oligomer carboxylic acid surfactants in perfiuorinaté liquids by ball milling or ultrasonic agitation at 
ambient conditions, was demonstrated by Kaiser $(1969,1973)$ over twenty years ago. Since its inception in 1985, ESI has been applying this chemistry to surface cleaning of high value equipment. In particular, ESI has demonstrated that small particles are more effectively removed from a variety of surfaces with dilute solutions of perfluorinated surfactant in perfluorinated liquids than with either CFC-113, or a perfluorinated liquid that does not contain any dispersing agent (Kaiser, 1987, 1989).

Cleaning tests have been run with a variety of fine particle contaminants and substrates. Particles examined have included fumed lead oxide, silica, ferric oxide, carbon black, and unknown dirt. The size of these particles ranged from fractions of a micron to ens of microns. Substrates that were examined have included glass (Pyrex), quartz, silicon, Teflon, polypropylene, epoxy resins, steel, stainless steel, beryllium and brass. Parts complexity ranged from flat plates to intricate devices, such as end housings of inertial guidance instruments. While ultrasonic agitation has been the source of shear in most of the work performed to date, some limited experiments in which the cleaning solutions were sprayed on the work piece at a low pressure (50 psi) were also performed.

Phasex Corporation of Lawrence, MA, and ESI recently completed Phase I of an Air Force sponsored SBIR program (AF 91-186) to develop new processes for precision parts cleaning to replace existing CFC-113 cleaning operations. The approach taken on this contract was to remove oils by supercritical fluid (Phasex) and to remove particles with fluorocarbon surfactant solutions (Gallagher, Krukonis and Kaiser, 1992). Phasex and ESI were awarded a Phase II program which started in June 1992.

In one series of tests in the Phase I program, glass microscope slides were contaminated with a fine quartz powder with a weight average particle diameter of 3.0 $\mu \mathrm{m}$. The slides were cleaned with different cleaning solutions, and then examined under a dark field microscope.

Figure 1.2(a) is a photomicrograph of an untreated particle contaminated slide. Figures 1.2(b), 1.2(c), and 1.2(d) respectively are photomicrographs of slides which were similarly contaminated and then sonicated twice in CFC-113, FC-77, a perfluorinated liquid manufactured by $3 \mathrm{M}$ Corporation, and a $1 \%$ solution of hexafluoropropylene oxide (HFPO) oligomer carboxylic acid, a perfluorinated surfactant, in FC-77. In all cases, most of the particles originally present had been removed. However, while one can easily discern the presence of a significant number of residual particles on the slides cleaned with CFC-113 and with FC-77, it is hard to discern any particles on the siide that had been cleaned with the fluorinated surfactant solution.

An order of magnitude increase in the rate and extent of particle removal was observed higher when inertial guidance instrument parts were ultrasonically cleaned in a dilute solution of HFPO carboxylic acid in FC-77 instead of CFC-113. Some 

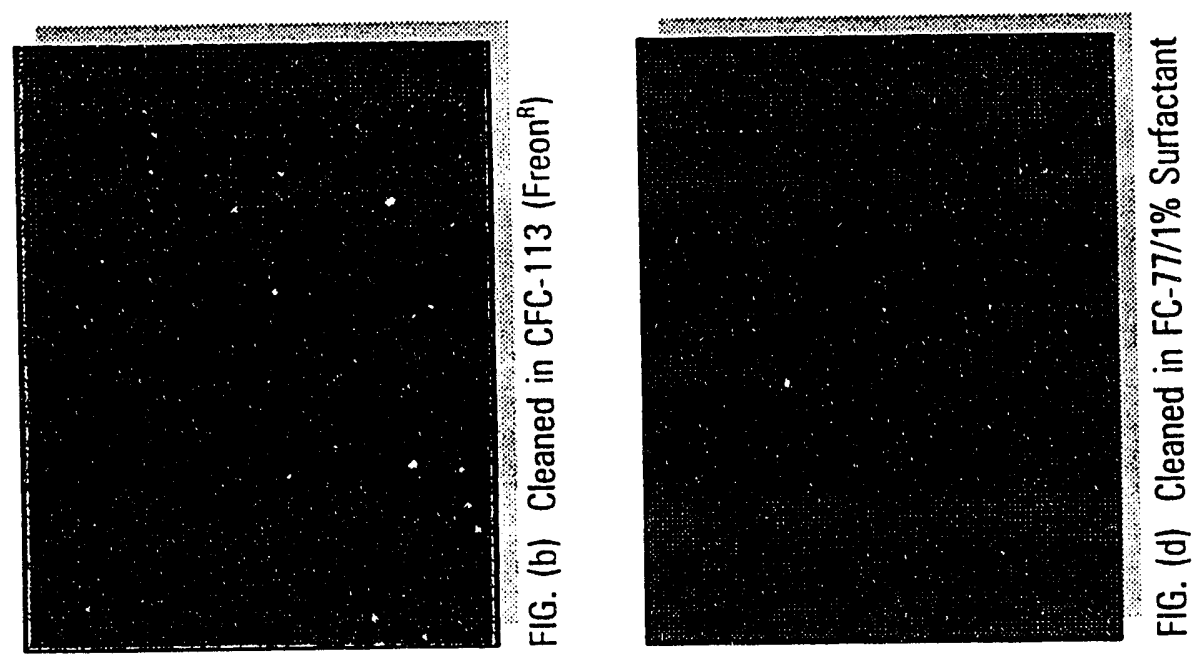

\section{$\frac{3}{8}$}
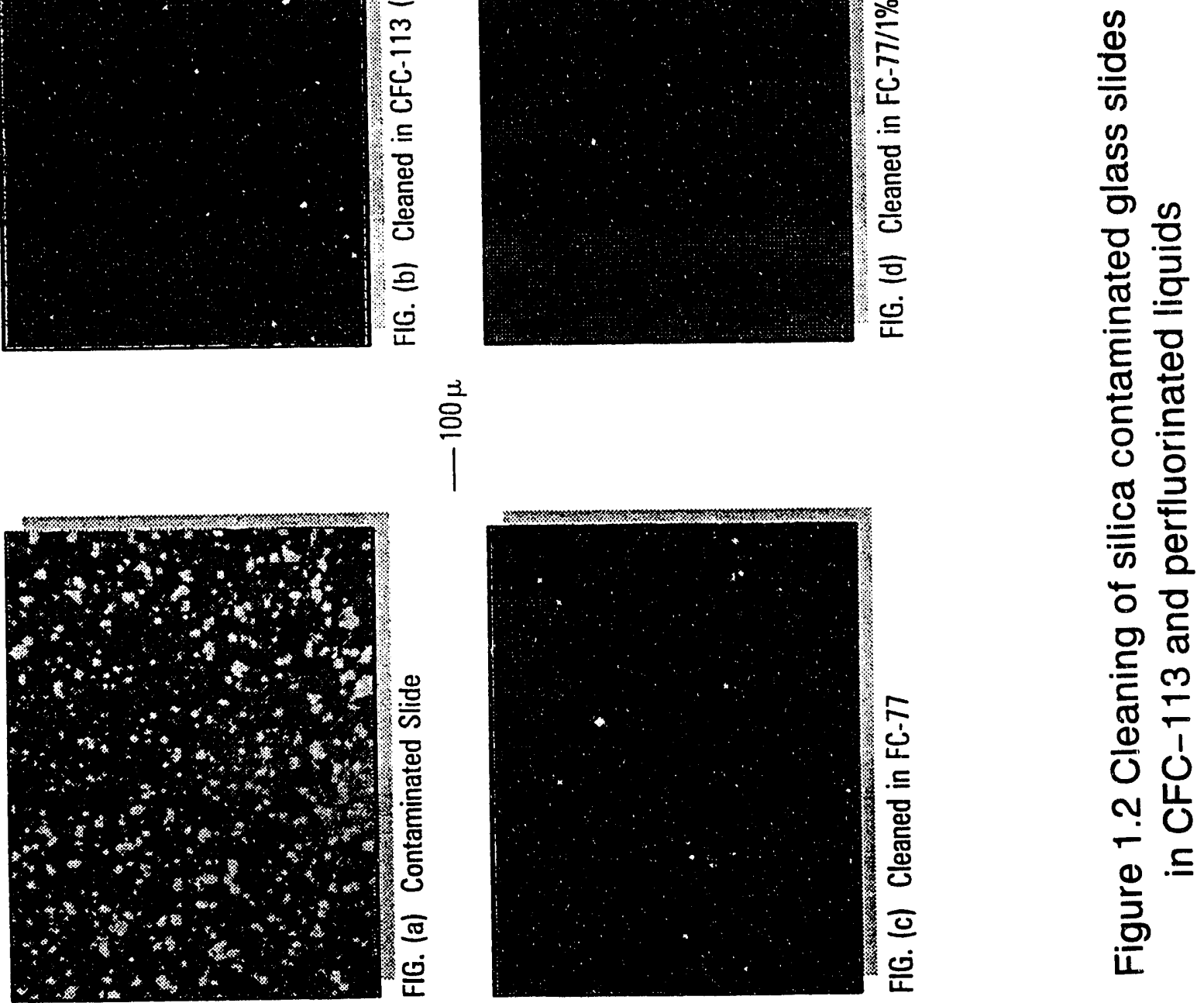
typical results for a single stage bellows used in an inertial guidance system are presented in Figure 1.3. This part was first cleaned seven times in CFC-113 before being cleaned in a $1 \mathrm{wt}-\%$ solution of HFPO carboxylic acid in FC-77. As can be seen in Figure 1.3, the number of particles removed in this sequence (Step 9) was not only twelve times higher than in the last cleaning step with CFC-113, but higher than the total number removed in the first sequence with CFC-113. Subsequent repeated cleaning with the fluorocarbon surfactant solution resulted in the continued removal of particles at a decreasing rate.

It has also been demonstrated that adsorbed HFPO carboxylic acid can be removed from metal washers and from instrument parts by simple extraction with a surfactant free fluorinated liquid. In these tests, while SIMS (secondary ion mass spectroscopy) and ESCA (electron spectroscopy for chemical analysis) measurements indicated the presence of insignificant, trace levels of HFPO carboxylic acid on extracted parts, these residual levels were below the detection limits of reflective angle infrared spectrometry or standard water break test, both of which could easily discern the presence of a monolayer of adsorbed surfactant.

\subsection{Research Issues to be Addressed - Program Objectives}

Nuclear contamination is inherently a surface phenomenon. Much nuclear waste is the result of the deposition of radioactive particles onto nonradioactive substrates. The presence of radioactive particles is responsible for all "smearable" contamination, and, if the radioactive particles are small enough, for some of the fixed contamination. Since radioactivity does not influence the physical chemistry of particle adhesion, the ESI process should be just as effective in removing radioactive particles as nonradioactive particles.

A key issue that needed to be addressed was measuring the residual number of particles that remained on a surface of a complex part after cleaning. The data presented in Figure 1.2 indicate complete removal of silica particles from flat plates. Such data are lacking for devices of complex shape which can not be examined by line of sight. The prior experiments with devices of complex shape measured the number of particles the cleaning liquid before and after contacting the device being surfaces of this part. The primary program objective was to demonstrate that radioactive particles could be quantitatively removed from complex parts by perfluorinated surfactant solutions, so as to decontaminate these parts. A corollary objective was to demonstrate that the rate and extent of particle removal, under comparable conditions of shear, is significantly greater in these solutions than in CFC-113 or surfactant free fluorocarbon liquids.

Another important goal was to demonstrate that radioactive particles suspended in the cleaning liquid could be quantitatively removed by simple filtration from this liquid in such a way that the radioactive particles could be simply disposed of and that the cleaning liquid could be recycled for further use. 


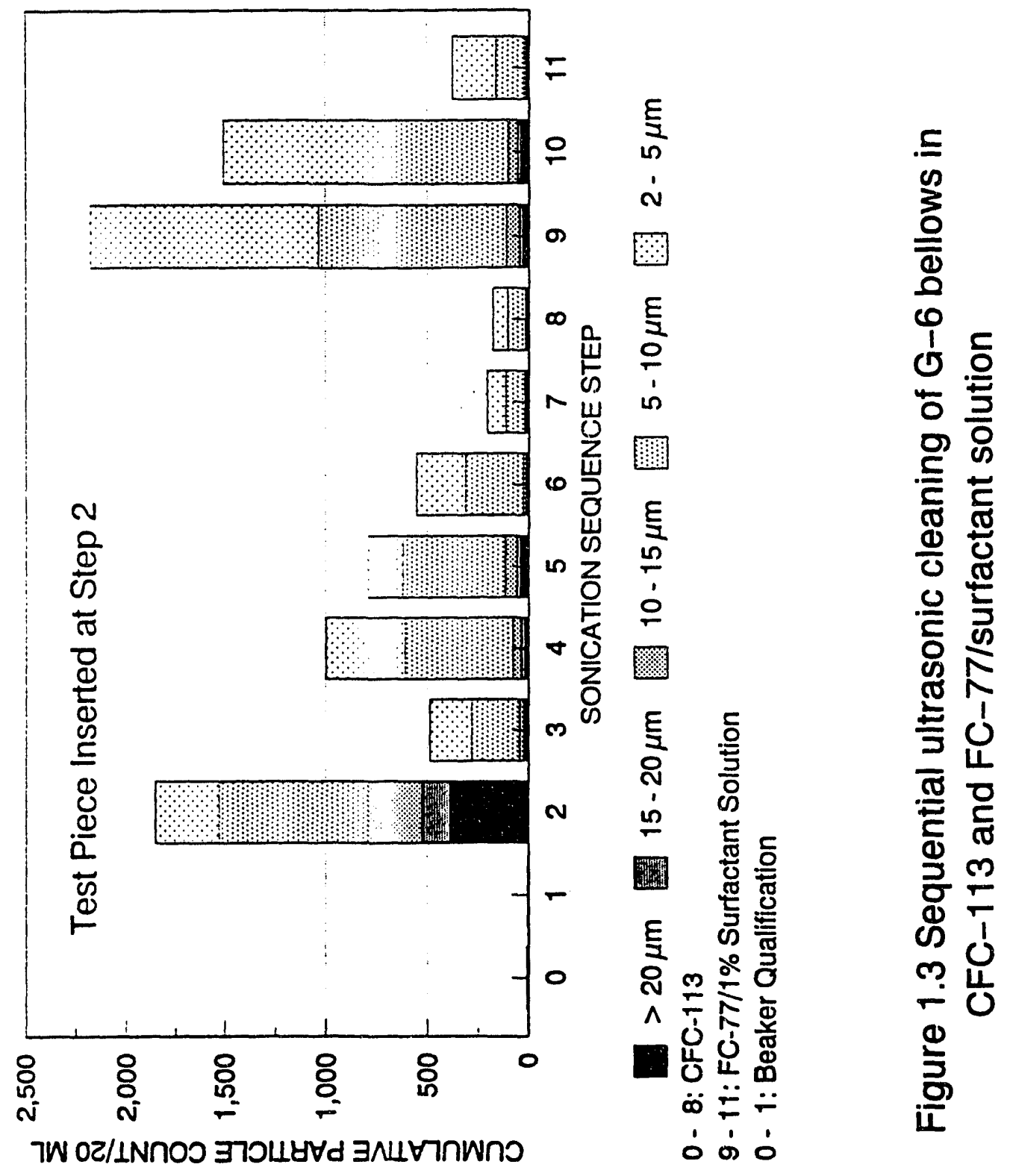


A major uncertainty with regards to fluid consumption is the effect of high energy radiation on the physical and chemical properties of the fluorinated surfactant solutions. While data were available on the radiation stability of Krytox 143, the composition of which is similar to that of the fluorocarbon tail of the fluorinated surfactant, Krytox 157FS, no data were available on the radiation stability of Krytox 157FS, or of solutions of this material in perfluorinated liquids. The presence of a carboxylic acid end group could render this HFPO oligomer more susceptible to radiation than the analogous fluorine endcapped oligomer. Determining the radiation stability of the process fluids, so as to establish that the fluorinated surfactant solutions used as the working media in the ESI particle removal/decontamination process exhibit sufficient radiation stability to allow them to be used effectively and economically under projected conditions of use, was therefore another major technical objective of the Phase I program.

The final goal of the program was to develop, based on the experimental data to be obtained in Phase I, as well as other ESI data, a design for a decontamination process that would be the basis of a prototype decontamination system that could be built, assembled and tested by ESI if it were awarded a Phase II program.

\subsection{Anticipated Benefits}

Successful implementation of the ESI process to nuclear decontamination would be of immense value to the nuclear industry and the U.S. government, and would definitely be of commercial value. The industry would be provided with a method and a system to remove undesirable radioactive particles from electronic and electrical equipment without damaging this equipment, and thus allowing it to be reused or disposed of as non-radioactive waste. Disposal costs have been increasing at an astronomic rate and will be several times higher than the current rate within the next decade.

In more general terms, the proposed research would be more than an environmentally suitable alternate for high pressure CFC-113 decontamination systems, since it would result in higher particle removal efficiencies. If the proposed process is as successful as anticipated, equipment which would otherwise be buried in a nuclear waste site would be available for reuse, with a resulting dual cost reduction: lower waste disposal costs, and lower capital replacement costs. 


\section{RADIATION RESISTANCE OF PROCESS LIQUID:}

\subsection{Introduction}

A major uncertainty with regards to the economical practicality of the proposed process is the radiation stability of the process fluids under likely conditions of use. The fluids have to be stable enough to be reused for many process cycles. While data are available on the radiation stability of perfluorinated liquids and of Krytox 143, the composition of which is similar to that of the fluorocarbon tail of the fluorinated surfactant, Krytox 157FS, as noted in the Introduction, no data are available on the radiation stability of Krytox 157FS, or of solutions of this material in perfluorinated liquids. The presence of a carboxylic acid end group could render this HFPO oligomer more sensitive to radiation than the analogous fluorine endcapped oligomer.

Determining the radiation stability of the process fluids was one of the major technical objectives of Phase I, and thus establish that these exhibit sufficient radiation stability to allow them to be used effectively and economically under projected conditions of use.

\subsection{Experimental Methodology}

\subsubsection{Materials}

The radiation stability of the following materials was evaluated:

1. Perfluoroheptane (3M 's PF 5070)

2. Poly(hexafluoropropylene oxide) carboxylic acid (Krytox 157FS-M, Lot 14, DuPont)

3. 1 wt-\% solution of Krytox 157FS-M in PF-5070

\subsubsection{Radiation Exposure}

Four $60 \mathrm{ml}$ samples of each of the three working liquids were prepared for irradiation in the spent fuel pool of the MTT Nuclear Reactor (MTTR2). All the samples were packaged in high density polyethylene bottles that were individually sealed in plastic bags. A radiochromic dye material was taped to each bottle to record the radiation exposure of the sample. The samples were exposed up to approximately $10^{\wedge} 7 \mathrm{R}$ of gamma radiation from fission products. The maximum dose rate in the pool is $3 \times 10^{\wedge} 5 \mathrm{R} / \mathrm{hr}$. Samples received dose rates ranging from approximately $0.5 \mathrm{Mrad}$ to $10 \mathrm{Mrad}$.

\subsubsection{Analytical Methods}

The following properties of irradiated samples were noted or measured, and compared to the properties of the unirradiated materials: visual appearance, presence of odor, 
density, viscosity at room temperature, ultraviolet adsorption, water extractable fluorides, $\mathrm{pH}$ of the water extract, and acid number.

Density was measured determining the weight on an analytical balance of the liquid contained in a calibrated pycnometer of known volume $(25 \mathrm{ml})$.

Viscosity was measured on a Brookfield Model LVT cone and plate viscometer with temperature control of $\pm 1^{\circ} \mathrm{C}$.

UV spectra were obtained on a Shimadzu Model $1201 \mathrm{UV} /$ vis spectrophotometer in quartz cells with high transmission at $200 \mathrm{~nm}$ (Hellma QS cells). Because of the high UV adsorption of the pure surfactant, these measurements were made in a demountable cell with a $0.2 \mathrm{~mm}$ path length. All other measurements were made with a standard $10 \mathrm{~mm}$ path length cell.

Hydrolyzable fluoride as \% of total fluoride was obtained by measuring the fluoride concentration of a water extract by ASTM Method D 1179-88. Extractions were performed by agitating $20 \mathrm{ml}$ samples of fluorinated liquid with $80 \mathrm{ml}$ of distilled water for 24 hours at room temperature. Fluoride ion concentration measurements were obtained with a Radiometer Model PHM 64 research pH meter fitted with a Radiometer Model FK 1502 F fluoride ion electrode. The $\mathrm{pH}$ of the extracts was obtained by replacing the fluoride ion electrode with a standard $\mathrm{pH}$ electrode (Radiometer Model GK 2401C combined electrode).

Acid number was measured by a modification of ASTM Methods D 664-89, in which CFC-113, because it dissolves Krytox 157FS and its solutions, replaced toluene in the titration solvent.

\subsection{Results}

The results of the radiation stability tests are summarized in Tables $2.1,2.2$, and 2.3 .

\subsection{Discussion of Results}

\subsubsection{Stability of PF-5070}

The results for PF-5070 are given in Table 2.1. The appearance and bulk properties (density and viscosity) of this material were essentially unchanged. A slight change in acid number, and noticeable changes were noted in hydrolyzable fluoride, $\mathrm{pH}$ of water extract, and UV adsorption at $200 \mathrm{~nm}$. Extractable fluoride increased from less than $1 \mathrm{ppm}$ for the non-radiated material to $52 \mathrm{ppm}$ for the material exposed to 9.4 


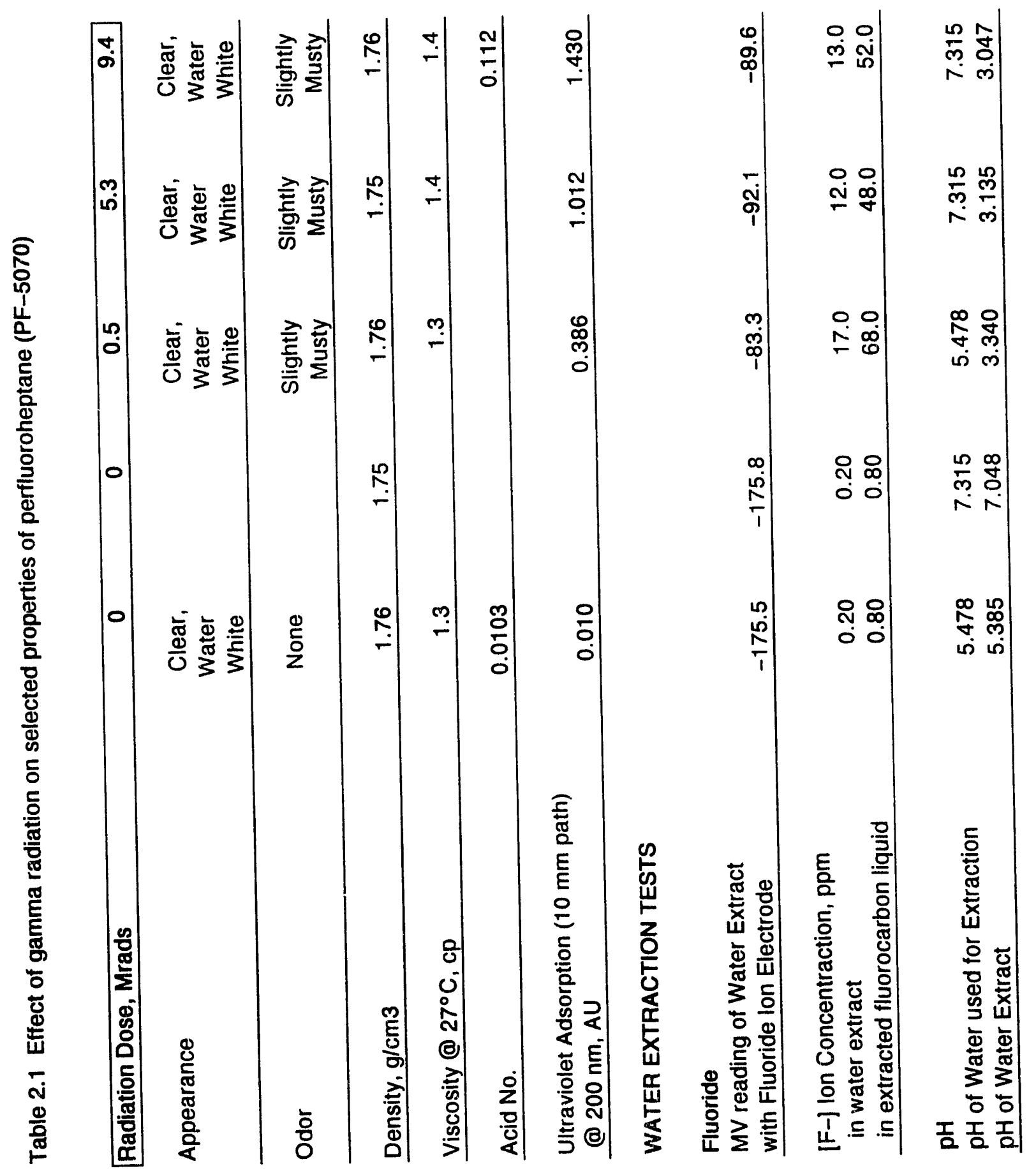




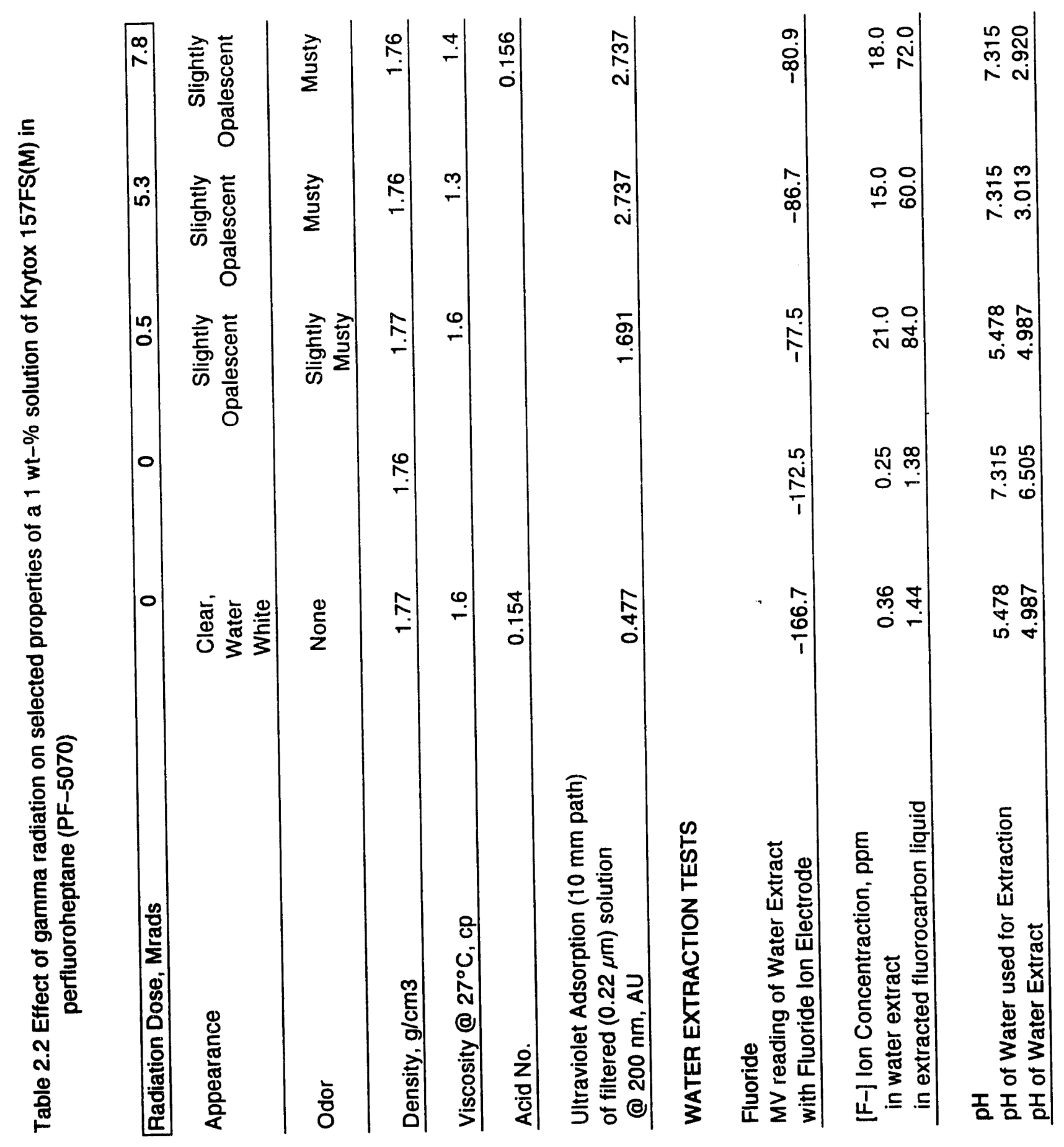




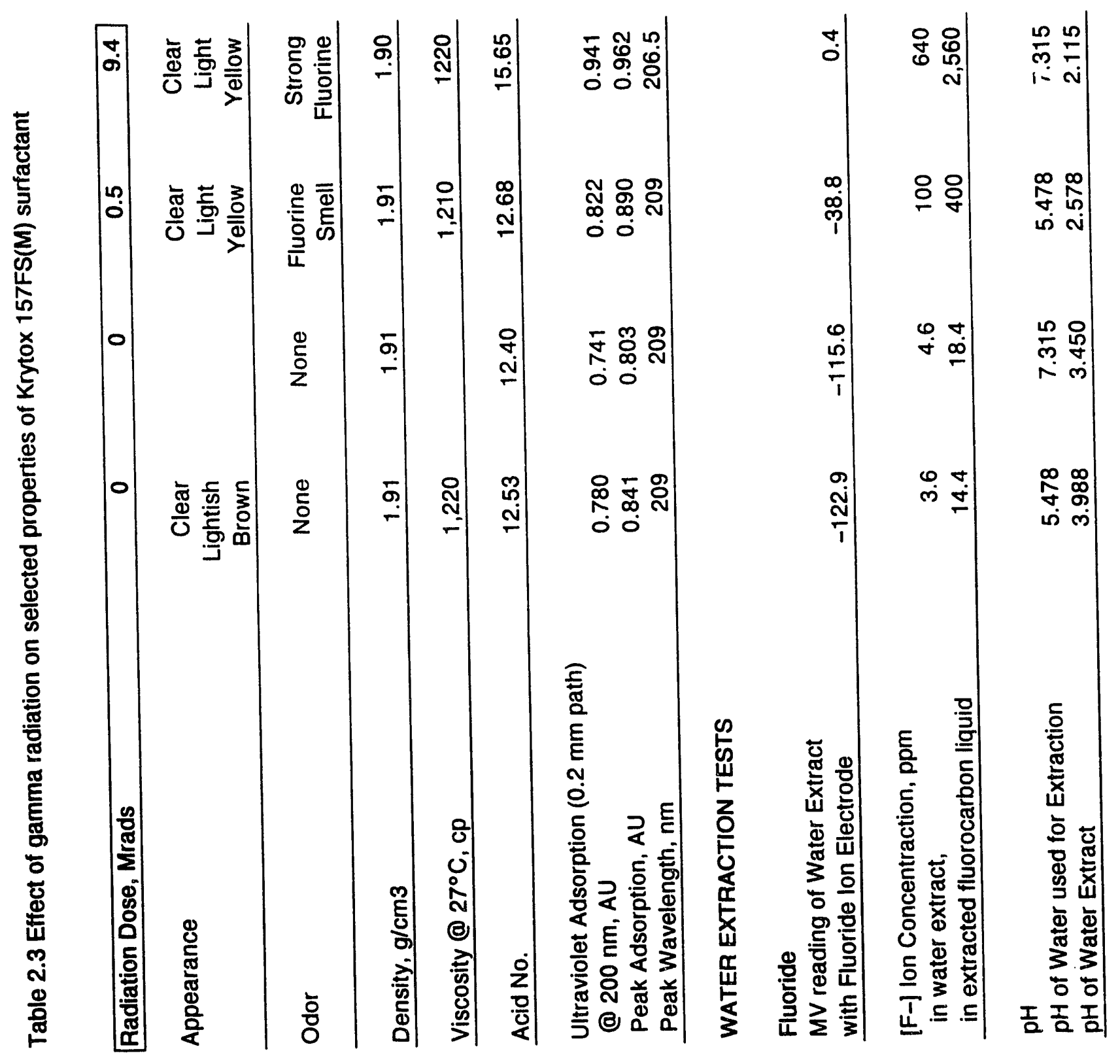


Mrad. The measurement of $68 \mathrm{ppm}$ noted for the material exposed to $0.5 \mathrm{Mrad}$ is believed to be erroneously high. The $\mathrm{pH}$ readings of the water extracts are commensurate with these values.

UV adsorption at $200 \mathrm{~nm}$ increased sharply with radiation exposure. This a sensitive measure of any change in the structure of the fluorinated liquid. Perfluorinated liquids are unusual in that they are transparent to short wave length, while most other organic materials are not. The increase in UV reading is believe to reflect radiation induced chemical reactions between PF-5070 and dissolved gases, such as oxygen.

\subsubsection{Stability of Krytox 157 FS}

The results for Krytox 157FS are given in Table 2.2. The bulk properties (density and viscosity) of this material were essentially unchanged. There was a slight change in color, and a 25\% increase in acid number and ultraviolet adsorption. Noticeable changes were noted in odor, hydrolyzable fluoride, and $\mathrm{pH}$ of the water extract.

Extractable fluoride increased from less than $20 \mathrm{ppm}$ for the non-radiated material to approximately $2,500 \mathrm{ppm}$ for the material exposed to $9.4 \mathrm{Mrad}$. The hexafluoropropylene oxide structure therefore seems more sensitive to gamma radiation than the than does a perfluoroalkane structure. The strong fluorine odor, and the $\mathrm{pH}$ value of 2.1 for the extraction water are further indications of the relatively higher hydrolyzable fluoride concentration.

The material used in these tests was as received from the supplier (DuPont Corp.). No attempt was made to purify this material which is known to contain metallic impurities, mainly colloidal iron, induced by the manufacturing operation. These impurities can be removed by extraction or filtration. The change in color from lightish brown to light yellow is believed due to reaction of this contaminant with radiation released fluorine atoms.

Irradiating to $9.4 \mathrm{Mrad}$ resulted in about a $25 \%$ increase in both ultraviolet adsorption at about $200 \mathrm{~nm}$ and in acid number. It is believed that these changes are both due to the incorporation of oxygen as $\mathrm{C}=\mathrm{O}$ groups into the hexafluoropropylene oxide structure. Since hardly any change in viscosity was observed, it is unlikely that there was any significant amount of radiation induced chain scission

\subsubsection{Stability of 1\% Solution of Krytox 157FS in PF-5070}

The results for Krytox 157FS/ PF 5070 solutions are given in Table 2.3. The bulk properties (density and viscosity) and acid number of this material were essentially unchanged. There was a slight change in appearance and odor. Noticeable changes were noted in hydrolyzable fluoride, $\mathrm{pH}$ of the water extract, and ultraviolet 
adsorption.

Values for the concentration of hydrolyzable fluoride and $\mathrm{pH}$ of the extraction water are slightly higher than the values obtained for pure PF-5070. The differences in fluoride concentration are about 1-3\% of the values noted for pure Krytox 157FS. This would indicate that the rule of mixtures applies in this case.

The irradiated solutions were slightly opalescent, which was an unexpected result. This haze was removed by passing the solution through a $0.22 \mu \mathrm{m}$ filter. It is believed that this haze is due to the presence of radiation induced reaction products of impurities present in the Krytox 157FS used to make up the test solutions. It is to be noted that these solutions were not filtered before being exposed to gamma radiation in order to simulate a worst case condition. This situation is not anticipated to occur in actual practice of the particle removal process which calls for careful filtration of all process fluids.

The ultraviolet adsorption of the solutions was significantly higher than that of pure PF-5070. This is due in mainly to the presence of surfactant which absorbs strongly in the $200-220 \mathrm{~nm}$ range of the spectrum.

\subsection{Conclusions}

While changes in the structure of PF-5070, Krytox 157FS, and solutions of Krytox 157FS in PF-5070 are sbserved under gamma irradiation, these do not appear to result in catastrophic damage to the liquids, even at the highest radiation levels used (10 Mrad). Radiation stability of the process liquids does not appear to be a show stopper in terms of the proposed decontamination application where time and volume averaged radiation exposure of the working fluids, even with badly contaminated parts, will be significantly less than the exposure values used in the evaluation tests. 


\section{DECONTAMINATION STUDIES}

\subsection{General Methodology}

Test pieces were contaminated by immersion in a suspension of radioactive iron oxide particles in isopropanol. After the alcohol evaporated, the radioactivity of the contaminated test piece was measured. The test piece was decontaminated by placing it in a clean container containing a given volume of cleaning liquid free of contaminating particles. The container was placed in an ultrasonic bath containing a coupling liquid (e.g. water), and sonicated for a short period of time $(5 \mathrm{~min})$. The supported test piece was then removed from the beaker, and its radioactivity was measured. The radioactivity of the test piece, and of the cleaning liquid in the container, before inserting the test piece in the cleaning solution and after its removal, are a quantitative measure of the amount of particle transfer from the test piece to the liquid, or decontamination, that occurred during the test.

The test piece was then placed in a new container containing fresh cleaning liquid, and again subjected to the test procedure described above. In prior work on the removal of nonradioactive particles, as indicated in Figure 1-3, it has generally observed that, with each sequential process step, additional particles are removed from the test piece, but that the number of particles removed and their size both tend to decrease. Test sequences were carried out until no further significant transfer of radioactive particles from the test piece to the cleaning liquid occurred.

The test piece was then saved for possible further testing, such as cleaning in another liquid, or for evaluation, such as microscopic examination.

\subsection{Experimental Procedure}

\subsubsection{Process Materials}

\subsubsection{Properties of Model Radioactive Contaminants}

Well characterized radioactive iron oxide ( $\mathrm{Fe} 2 \mathrm{O} 3$ ) powajers were used as model contaminants in the Phase I decontamination tests. The reasons for choosing this material include:

a. The $\mathrm{Fe}(59)$ isotope, created by thermal neutron activation, has a half-life of 44.5 days. This half life is of the same duration as the elapsed time of the experimental program that was performed. However, it is not so long as to create significant and costly post-experimental disposal problems. The radiation from $\mathrm{Fe}(59)$ is conveniently measurable. 
b. Pigment grade iron oxides of high purity are available commercially in a range of particle sizes of interest.

c. Radioactive iron oxide (rust) is not an uncommon contaminant in operational facilities.

A grade of iron oxide powder that had been air classified into two fractions was obtained from the Donaldson Company of Minneapolis, MN. The particle size distribution of the feed and classified fractions of this material are presented in Figure 3.1. The particle size distributicn of these powders was performed by Coulter counter by the supplier. The particle size distribution of the feed material ranged from less than $1 \mu \mathrm{m}$ to $40 \mu \mathrm{m}$, with a weight average particle size of $11.9 \mu \mathrm{m}$. The particle size distribution of the fine fraction ranged from about $0.5 \mu \mathrm{m}$ to $2.4 \mu \mathrm{m}$, with a weight average particle size of $1.3 \mu \mathrm{m}$. The particle size distribution of the coarse fraction ranged from about $2.4 \mu \mathrm{m}$ to 40 $\mu \mathrm{m}$, with a weight average particle ize of $12.5 \mu \mathrm{m}$. Because of the dispropont tonate influence of large particles on the weight average diameter of a powder, the average particle diameters of the feed and of the coarse fractions are similar.

The composition of these iron oxide powders was determined by Instrumental Neutron Activation Analysis (NAA) at the MIT Nuclear Reactor Laboratory (MTT: $: 2 L$ ). The INAA results, based on the irradiation conditions given in Table 3.1 , are presented in Table 3.2 where the components of the powders are listed in order of decreasing concentration. These powders did not contain any significant amount of elements that could form hazardous or long lived isotopes.

Decontamination tests were performed with both the feed material and the fine fraction as the model contaminant after these had been irradiated for approximately one day in the MITR2. Irradiation conditions and radiation characteristics of these materials are given in Table 3.3.

\subsubsection{Cleaning Liquids}

Three cleaning liquids were used in the study:

a. CFC-113 as a baseline,

b. perfluoroheptane (3M's PF-5070),

c. a $1 \mathrm{wt}-\%$ solution of Poly (hexafluoropropylene oxide) carboxylic acid [DuPont's Krytox 157FS(M), Lot 14] in $\mathrm{P}^{-} 5070$.

The properties of these liquids were discussed in the previous chapter. 


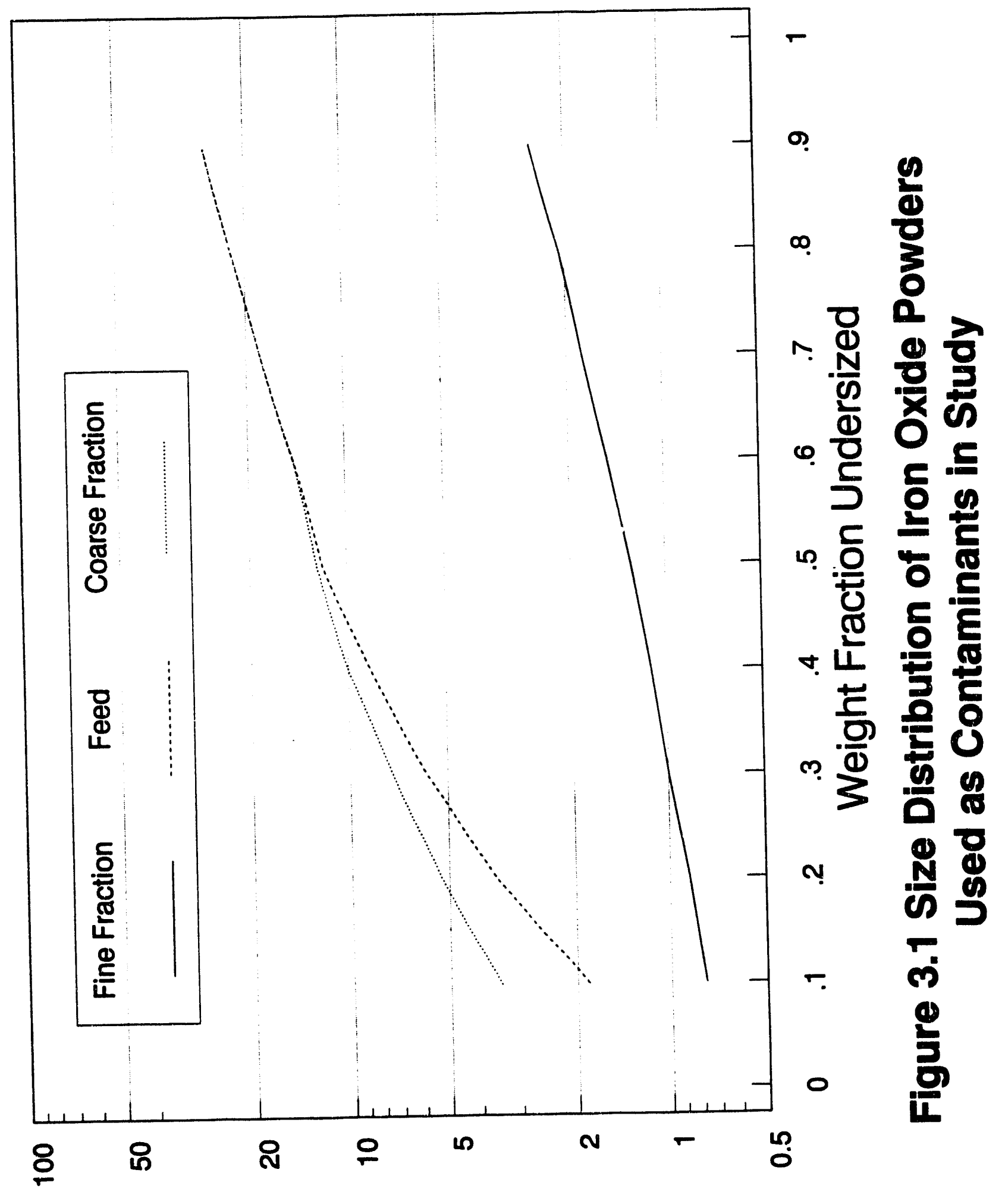

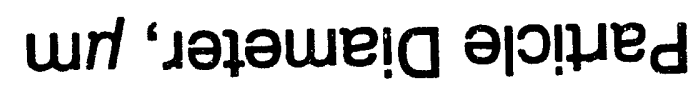


Table 3.1 Neutron irradiation for analysis of iron(+3) oxide powders used in cleaning experiments

CONDITIONS

$\begin{array}{lr}\text { LENGTH OF IRRADIATION } & 2 \mathrm{hrs} 27 \mathrm{~min} \\ \text { FLUX, NEUTRONSISEC } & 6.53 E+12 \\ \text { REACTOR POWER, MW } & 4.0 \\ \text { END OF IRRADIATION } & \\ \text { DATE } & 11 / 17 / 92 \\ \text { TIME } & 10: 51 \mathrm{AM} \\ \text { IRRADIATION POSITION } & 1 \mathrm{PH} 1\end{array}$

DOSE @ $10 \mathrm{~cm}$ DISTANCE ON 11/25/92@ 13:45

\begin{tabular}{|llll}
\hline SAMPLE FRACTION & COARSE & FINE & FEED \\
\hline
\end{tabular}

SAMPLE WEIGHT, GR

$0.02861 \quad 0.02751$

0.02941

DOSE RATE, MR/HR

0.35

0.4

0.35

SPECIFIC DOSE RATE, MR/GR.HR

12.2

14.5

11.9 
Table 3.2 Composition of iron( +3 ) oxide powders used in cleaning experiments as measured by instrumental neutron activation analysis

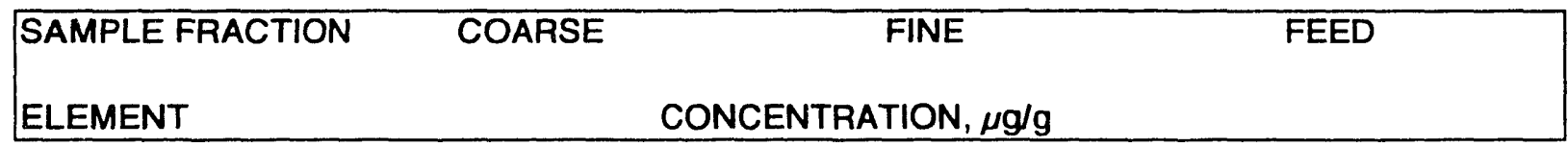

\begin{tabular}{|c|c|c|c|}
\hline $\mathrm{Fe}$ & $610,000 \pm 20,000$ & $690,000 \pm 30,000$ & $650,000 \pm 30,000$ \\
\hline $\mathrm{Cr}$ & $125 \pm 3$ & $97 \pm 2$ & $125 \pm 3$ \\
\hline $\mathrm{Ba}$ & $33 \pm 17$ & $145 \pm 21$ & $58 \pm 18$ \\
\hline$Z n$ & $31 \pm 4$ & $97 \pm 11$ & $34 \pm 5$ \\
\hline $\mathrm{Na}$ & $25 \pm 8$ & $55 \pm 8$ & $30 \pm 5$ \\
\hline As & $15 \pm 2$ & $53 \pm 5$ & $23 \pm 2$ \\
\hline $\mathrm{Ce}$ & $13 \pm 1$ & $46 \pm 2$ & $21 \pm 1$ \\
\hline La & $7.6 \pm 0.5$ & $26 \pm 2$ & $11 \pm 1$ \\
\hline Nd & $4.5 \pm 0.7$ & $18 \pm 2$ & $7.8 \pm 1.0$ \\
\hline Co & $3.8 \pm 0.2$ & $14 \pm 1$ & $6.0 \pm 0.3$ \\
\hline Sc & $3.1 \pm 0.2$ & $7.1 \pm 0.5$ & $3.8 \pm 0.2$ \\
\hline $\mathrm{Sm}$ & $1.1 \pm 0.1$ & $3.9 \pm 0.3$ & $1.7 \pm 0.1$ \\
\hline $\mathrm{Au}$ & $1.0 \pm 0.1$ & $0.78 \pm 0.03$ & $0.95 \pm 0.06$ \\
\hline $\mathrm{Sb}$ & $0.93 \pm 0.08$ & $2.4 \pm 0.2$ & $1.3 \pm 0.1$ \\
\hline $\mathrm{Yb}$ & $0.55 \pm 0.09$ & $1.4 \pm 0.2$ & $0.76 \pm 0.12$ \\
\hline $\mathrm{Br}$ & $0.53 \pm 0.30$ & $0.75 \pm 0.31$ & $0.68 \pm 0.27$ \\
\hline $\mathrm{Se}$ & $0.53 \pm 0.30$ & $2.5 \pm 0.4$ & $1.6 \pm 0.5$ \\
\hline Eu & $0.51 \pm 0.06$ & $1.4 \pm 0.2$ & $0.70 \pm 0.08$ \\
\hline Lu & $0.12 \pm 0.03$ & $0.26 \pm 0.06$ & $0.13 \pm 0.03$ \\
\hline
\end{tabular}


Table 3.3 Neutron irradiation of iron $(+3)$ oxide powders used in cleaning experiments

\begin{tabular}{|lll}
\hline SAMPLE FRACTION & FINE & FEED \\
\hline
\end{tabular}

Sample weight, gr

Length of irradiation, hrs

Flux, neutrons/sec

Reactor power, MW

End of irradiation

Date

Time

Irradiation position

Approximate gamma activitity @ $10 \mathrm{~cm}\left({ }^{*}\right), \mathrm{mr} / \mathrm{hr}$

Principal isotope

$\left({ }^{*}\right)$ at time of decontamination tests
$0.1 \quad 0.1$

$24.00 \quad 29.40$

$6.90 E+12 \quad 6.53 E+12$

44

$2 / 10 / 93 \quad 2 / 06 / 93$

08:42 00:29

$1 \mathrm{PH} 1 \quad 1 \mathrm{PH} 1$

$0.3 \quad 0.3$

$\mathrm{Fe}-59 \quad \mathrm{Fe}-59$ 


\subsubsection{Dispersion Liquid for Contaminating Solution}

Isopropanol was chosen as the dispersion medium to prepare the suspensions of radioactive iron oxide used to contaminate the test pieces. This material was chosen because it is a good dispersing medium for fine particles, evaporates without leaving a nonvolatile residue, and is reasonably safe to use in small quantities. It is a better dispersing medium than either PF-5070 or CFC-113, but not as good as a perfluorinated surfactant solution, which of course could not be used to prepare the contaminating solutions because this would invalidate the study.

\subsubsection{Test Pieces}

Decontamination tests were performed on flat coupons, nuts and bolts, test assemblies of washers bolted together, and on a test container that had been contaminated as a result of its use in a prior test. Other than for the container, the test samples were attached to either:

a. a metal post soldered to a mating metal cap, as also shown in Figure 3.2, or

b. a polypropylene post attached to a somewhat larger cap that would also seal the can during a decontamination test, as shown in Figures 3.3, 3.4, and 3.5 .

The reason for using these supports is that they allowed the test samples to be supported during test, and to be easily $t$ insferred from one vessel to another, for either contamination, decontaminati n or radiation detection.

The test pieces that were used are described below:

Type SG: This test piece consisted of a single aluminum washer supported between an $8 / 32$ nylon nut and the head of an $8 / 32 \times 1 / 2$ in long nylon bolt attached to a polypropylene support post, as shown in Figure 3.3. Screw threads were filled with silicone sealant. The composition and dimensions of the washer were as follows:

Material: Type 1100 Aluminum Alloy, Internal Diameter: 0.169 in $(0.429 \mathrm{~cm})$

External Diameter: 1.000 in $(2.540 \mathrm{~cm})$

Thickness: 0.031 in $(0.079 \mathrm{~cm})$ 


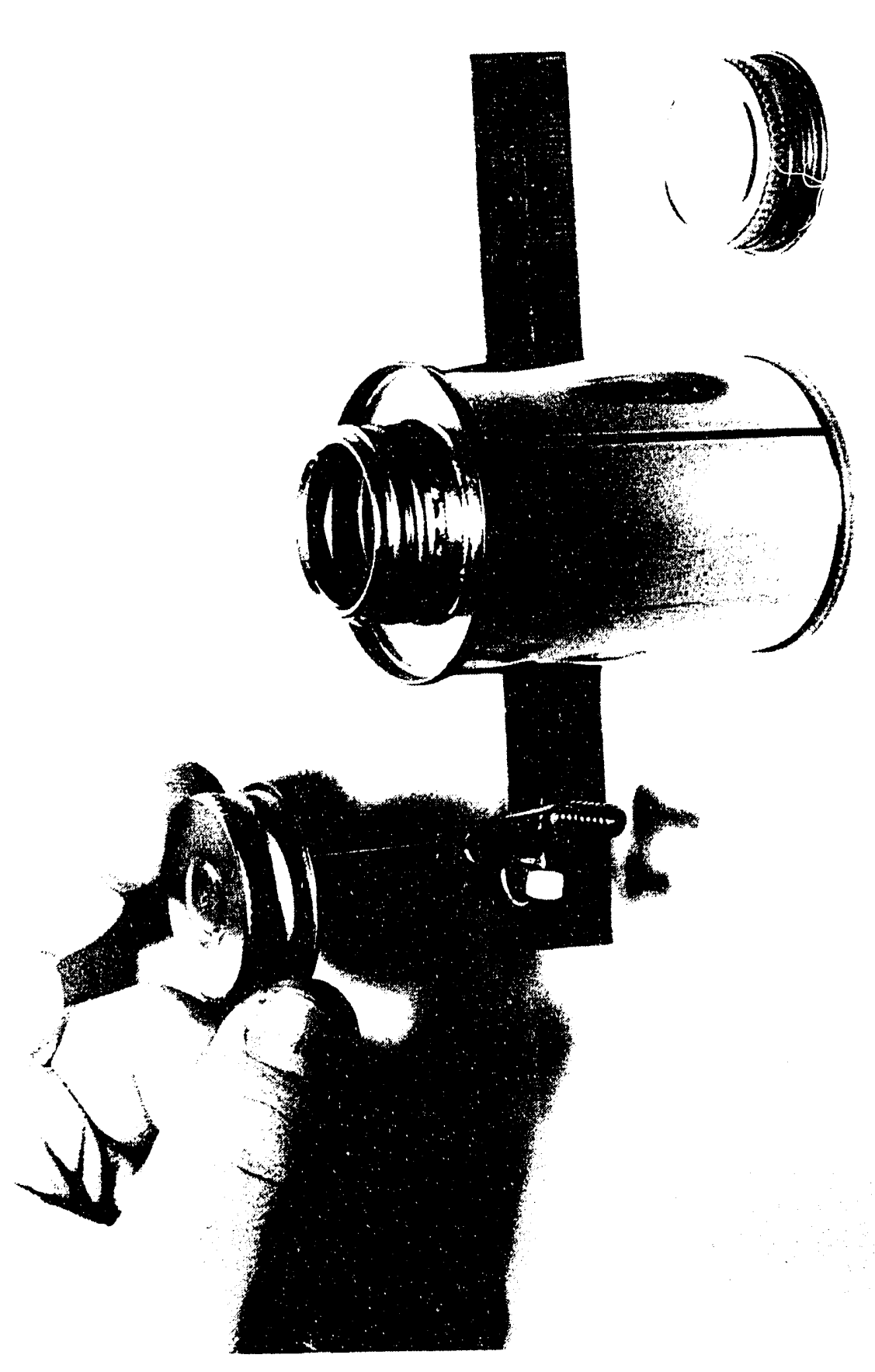

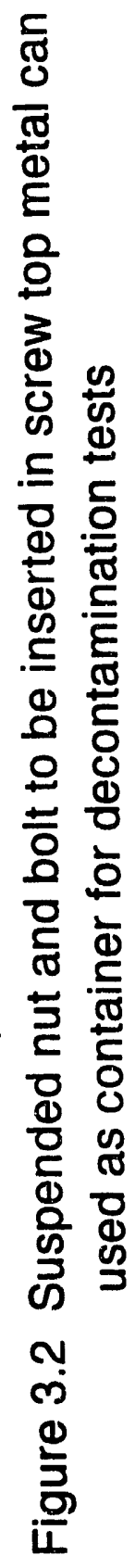




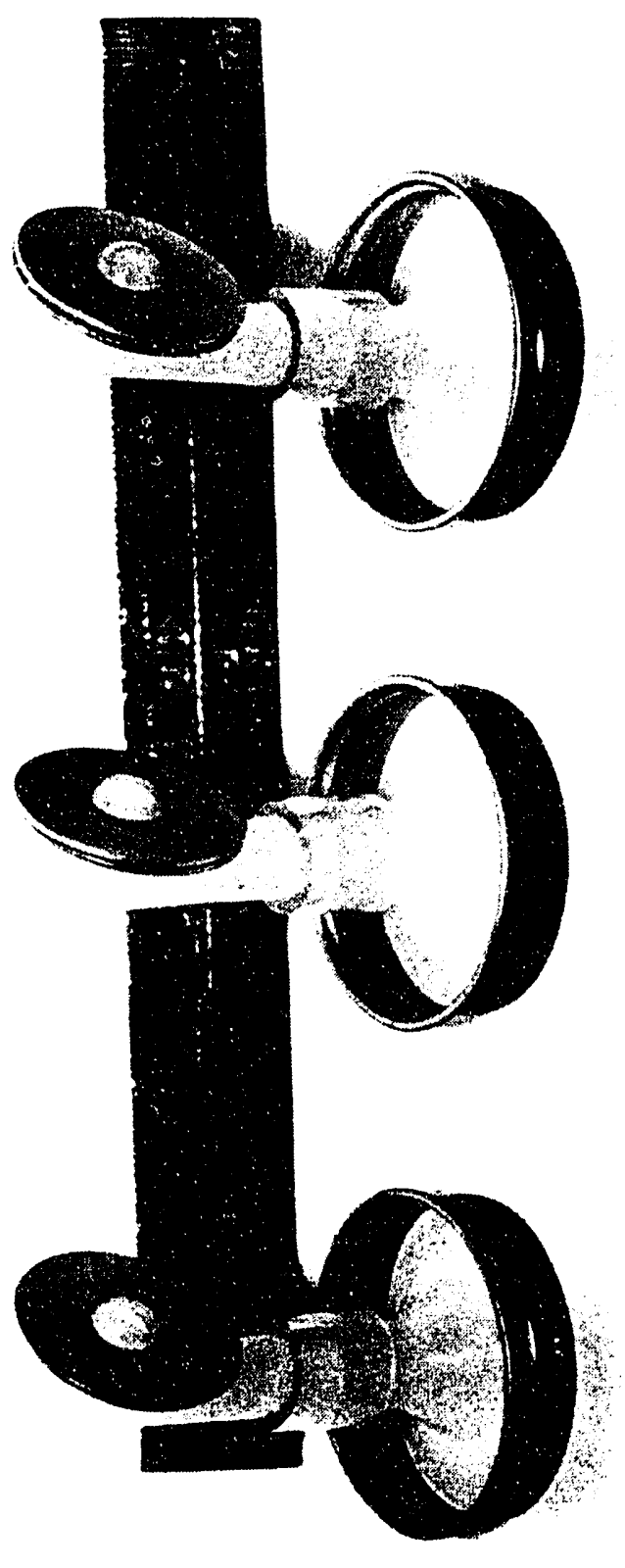

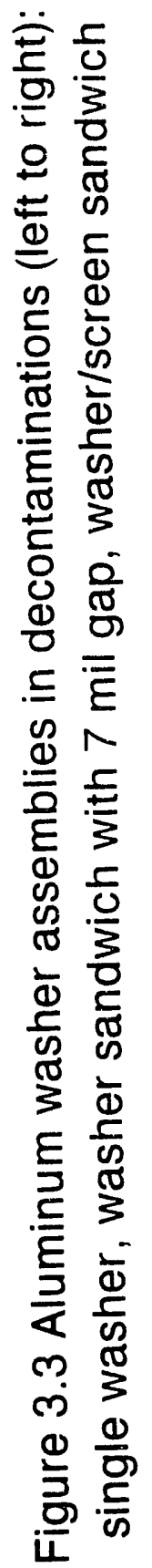




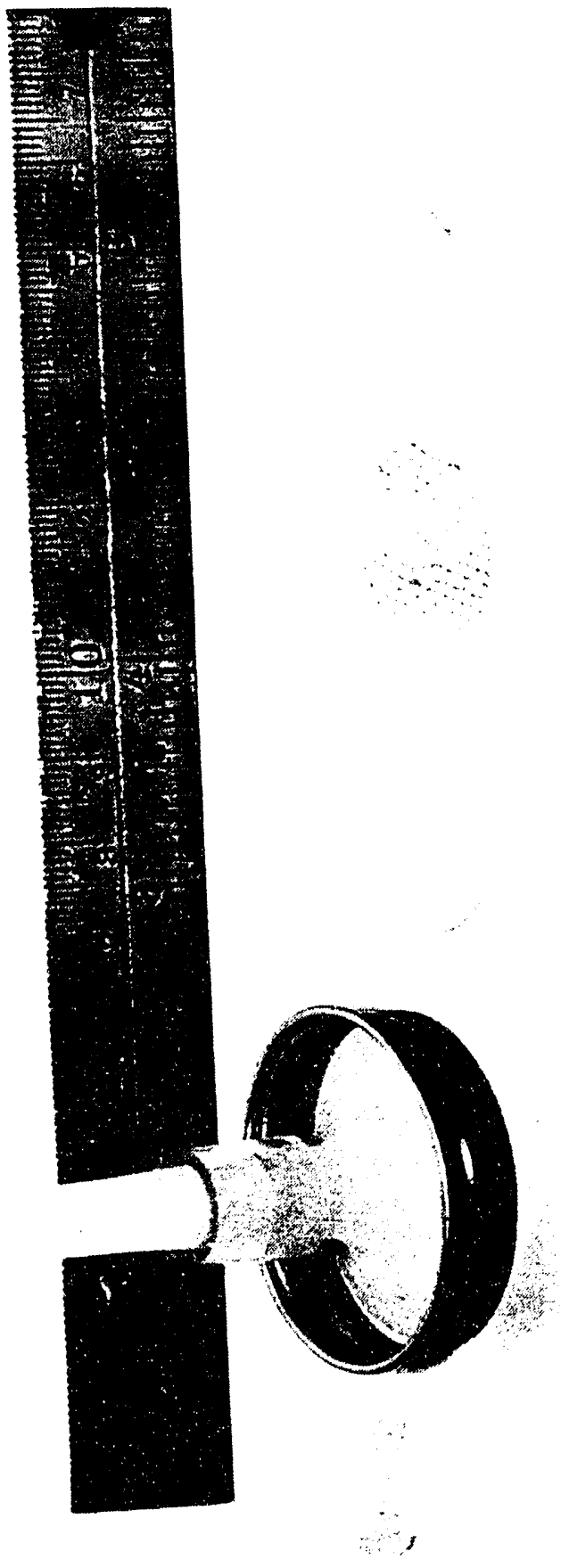

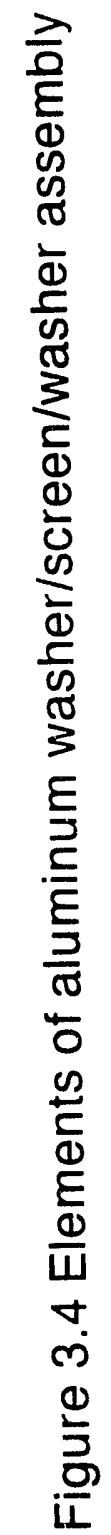




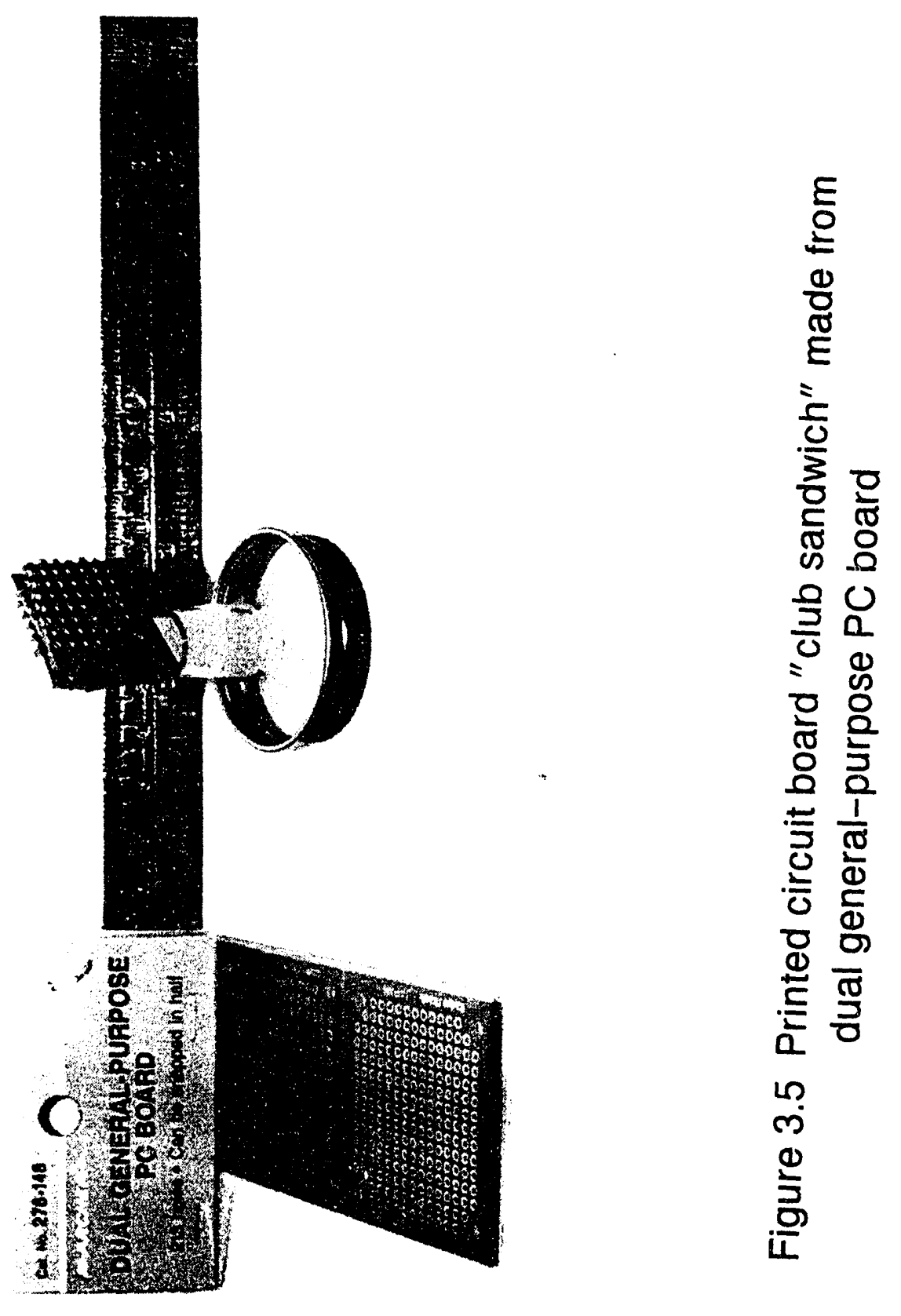




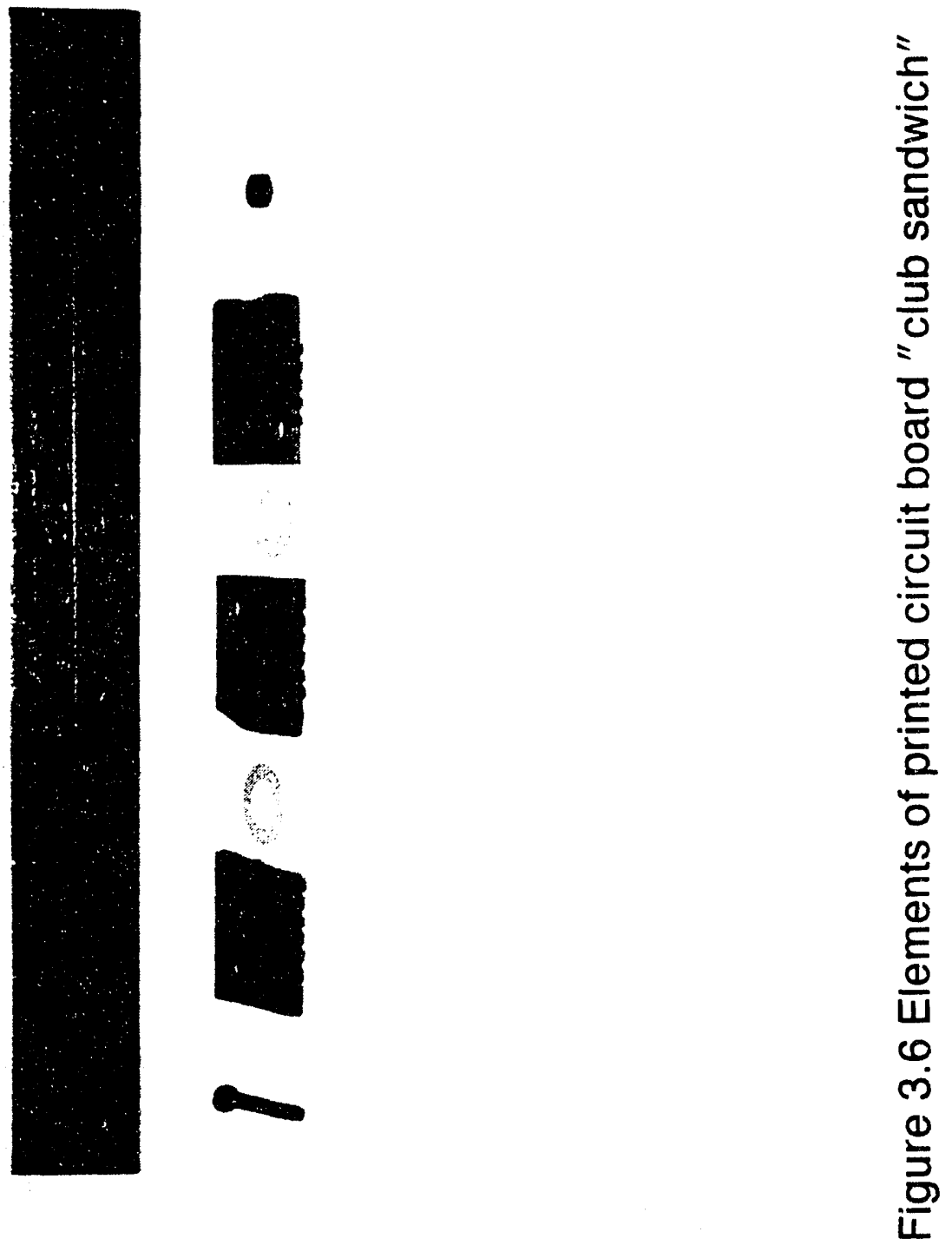


Type 2G: This test piece consisted of an aluminum washer sandwich, as shown in Figure 3.3. The arrangement is similar to that used for Type SG, except that the single aluminum washer is replaced by an assembly of two aluminum washers separated by a small, thin stainless steel washer. The composition and dimensions of the spacer washer were as follows:

Material: Type 304 Stainless Steel Alloy, Internal Diameter: 0.273 in $(0.693 \mathrm{~cm})$

External Diameter: 0.457 in $(1.161 \mathrm{~cm})$

Thickness: 0.002 in $(0.005 \mathrm{~cm})$

Type D7: This test arrangement is similar to that used for Type 2G, except that a thicker stainless steel steel washer is used as a spacer. The aluminum washers were also roughened with 220 grit emery cloth, and the screw threads were not sealed with silicone polymer. The composition and dimensions of the spacer washer were as follows:

Material: Type 304 Stainless Steel Alloy, Internal Diameter: 0.253 in $(0.643 \mathrm{~cm})$ External Diameter: 0.373 in $(0.947 \mathrm{~cm})$ Thickness: 0.007 in $(0.018 \mathrm{~cm})$

Type SS: This test arrangement is similar to that used for Types $2 \mathrm{G}$ and D7, except that the thin stainless steel spacer washer is replaced by a piece of 10 mesh aluminum screen cut to the same dimensions as the external aluminum washers, as shown in Figures 3.3 and 3.4. The diameter of the screen wire was measured to be 0.010 in $(0.024 \mathrm{~cm})$. The overall thickness of the sandwich was approximately 0.089 in $(0.226 \mathrm{~cm})$, which corresponds to a screen thickness of 0.027 in $(0.069$ $\mathrm{cm})$. As with type D7, the aluminum washers were also roughened with 220 grit emery cloth, and the screw threads were not sealed with silicone polymer.:

Type PC: This test arrangement is a "club sandwich" of three approximately 1 in $(2.54 \mathrm{~cm})$ square pieces of general purpose circuit board separated by 2 mil $(0.005 \mathrm{~cm})$ stainless steel washers, fastened to each other, and to a polypropylene support post by a $2 / 56 \times 1 / 2$ in steel bolt and two $2 / 56$ steel nuts. The assembly is shown in Figure 3.5, and the separate components in Figure 3.6. The circuit board is made of fiber glass reinforced epoxy. A $4.5 \mathrm{~cm} \times 9 \mathrm{~cm}$ piece of material contains 213 holes approximately $1 / 32$ in $(0.08 \mathrm{~cm})$ in diameter at a pitch of 11 holes to the inch ( 4.3 holes to the $\mathrm{cm}$ ). The periphery of each hole on the top side of the board is copper plated.

Type $N \& B$ : This test arrangement consists of a $1 / 4 / 20 \times 1 / 2$ in long stainless steel bolt and of a 1/4/20 stainless steel nut hung from a wire support, as shown in Figure 3.2. ESI has used this arrangement as a model configuration in the instrument part cleaning studies being performed for the U.S. Air Force under 
Contract F 01701-92-C-0017. This arrangement was included in the present test program to compare the effect of different process liquids on the removal of nonradioactive particles, as measured by optical counters, to the removal of radioactive particles.

Test Can: This test arrangement was the test container from a prior decontamination run in which CFC-113 was used as the decontaminating liquid, and which was found to be highly radioactive because of settling of radioactive particles in the can before removal of the CFC-113. This presented an opportunity to evaluate the effectiveness of the proposed technology to a real decontamination situation.

\subsubsection{Experimental Equipment and Procedure}

\subsubsection{Test Vessels}

The contamination of test samples and their subsequent decontamination experiments were performed in $4 \mathrm{oz}$ round, cone top steel cans with a 1 in (2.54 $\mathrm{cm}$ ) screw neck, as shown in Figure 3.2. These cans were used because the experimental procedures could be performed without spattering radioactive suspensions during sonification.

\subsubsection{Contamination Procedure}

Except for some of the initial tests where no ultrasonic agitation was used, the test pieces were contaminated by immersion in a suspension of 0.1 grams of radioactive iron oxide powder in $100 \mathrm{ml}$ of isopropanol. To attain a good level of powder deposition, the suspension was first agitated ultrasonically for $5 \mathrm{~min}$. After stopping the agitation, the test piece was then dipped into the suspension about 5 times within a period of about $1 \mathrm{~min}$. The test piece was then immersed in the suspension while sonicating for $3 \mathrm{~min}$. The test piece was then withdrawn from the suspension and allowed to air dry before its radiation emissions were measured.

\subsubsection{Decontamination Procedure}

The decontamination tests involved filling the test containers to within $28 \mathrm{~mm}$ to $32 \mathrm{~mm}$ of their top with decontamination test liquid. These heights correspond to approximately $120 \mathrm{ml}$ to $105 \mathrm{ml}$ of liquid. The volume of decontamination liquid was increased with each subsequent decontamination step so as to assure that any material immersed in a prior step would be immersed in a subsequent one. The decontamination liquid was then degassed by preheating the liquid to within $10^{\circ} \mathrm{C}$ of its boiling point, and then sonicating it in the ultrasonic bath used to perform the decontamination tests, and which is described further below.

The supported test sample was then placed in the container, and which was, in 
turn placed in a water filled Crest Model HT-710-3 ultrasonic bath. The bath is approximately 7 in $(18 \mathrm{~cm})$ wide by 10 in $(25 \mathrm{~cm})$ long by 10 in $(25 \mathrm{~cm})$ deep, and has a fluid capacity of approximately 3 gallons (12 liters). The bath is heated, and its temperature can be maintained to within $\pm 1{ }^{\circ} \mathrm{C}$, up to $120^{\circ} \mathrm{C}$. Most tests were performed at $40^{\circ} \mathrm{C}$. The bath is powered by a Model 4PI-250-3 ultrasonic generator which generated $40 \mathrm{kHz}$ waves plus complex resonances at 50 to $90 \mathrm{kHz}$, with a rated full wave average power output of 250 watts. -

\subsubsection{Radiation Detection}

The amount of radioactive iron oxide present on a test sample was measured by counting the gamma radiation emitted when the test sample of reproducible geometry was placed near a scintillation counter with a 3 in $\times 3$ in $(7.5 \mathrm{~cm} \times 7.5$ $\mathrm{cm}$ ) sodium iodide crystal scintillation detector. Two different detection equipment configurations duririg the test program. These configurations are described in Table 3.4. The first configuration was used on tests performed up to Feb. 18, 1993; the second configuration was used on all subsequent tests. The second configuration provided a five fold gain in signal to noise ratio. With the second configuration, background noise decreased by an order of magnitude, from approximately 1,200 to 1,400 counts per 100 seconds to 160 to 180 counts per 100 seconds, while counting efficiency decreased by about $60 \%$ from 0.485 counts / decay to 0.19 counts / decay.

The detector was contained in a shielded chamber that was large enough to accommodate the $4 \mathrm{oz}$ vessels in which the experimental work was being performed. It was therefore possible to measure the radiation characteristics of either the test sample supported in an unused, clean vessel, or the decontamination liquid in its test vessel.

\subsection{Experimental Results}

Experimental results are summarized in Tables 3.5 to 3.11 .

Table 3.5 presents summary results for the decontamination experiments performed with a single aluminum washer and aluminum washer sandwiches. Table 3.6 presents the same data in terms of percent decontamination achieved.

Table 3.7 presents summary results for the decontamination experiments performed with aluminum washer screen sandwiches. Table 3.8 presents the same data in terms of percent decontamination achieved.

Table 3.9 presents summary results for the decontamination experiments performed with nuts and bolts and the contaminated test can. Table 3.10 presents the same data in terms of percent decontamination achieved.

Table 3.11 presents summary results and the percent decontamination achieved for 
Table 3.4 Configuration of radiation detection equipment used in study

\begin{tabular}{|c|c|c|}
\hline Configuration & 1 & 2 \\
\hline Detector & $3 \times 3$ in Nal Crystal & $3 \times 3$ in Nal Crystal \\
\hline \multicolumn{3}{|l|}{ Shielding } \\
\hline Material & Lead & Lead \\
\hline Thickness, in & 3 & 3 \\
\hline \multicolumn{3}{|l|}{ Power Supply } \\
\hline Manufacturer & Mechtronics & Mechtronics \\
\hline Model & High Voltage Supply & High Voltage Supply \\
\hline Voltage, $\mathrm{V}$ & 1,100 & 1,100 \\
\hline \multicolumn{3}{|l|}{ Linear Amplifier } \\
\hline Manufacturer & Hamner & \\
\hline Model & NA 11 & \\
\hline Gain & $0.5 \times 1.0$ & \\
\hline Polarity Switch & Inverted & \\
\hline \multicolumn{3}{|l|}{ One Channel Analyzer } \\
\hline Manufacturer & Tennelec & \\
\hline Model & TC-405 & \\
\hline Discriminator Settiris $v$ & 1.0 & \\
\hline \multicolumn{3}{|c|}{ Combined Amplifier/One Channel Analyzer } \\
\hline Manufacturer & & Mechtronics \\
\hline Model & & \\
\hline Gain & & $512 \times 1.9$ \\
\hline Discriminator Setting, $\mathrm{V}$ & & 3.0 to 6.0 \\
\hline \multicolumn{3}{|l|}{ Counter/Timer } \\
\hline Manufacturer & Camberra & Camberra \\
\hline Model & 1772 & 1772 \\
\hline Counting Efficiency, counts/decay & 0.485 & 0.190 \\
\hline Fe-59 Gamma Ray Peaks, Volts & $6.4,5.5$ & $5.8,5.0$ \\
\hline
\end{tabular}




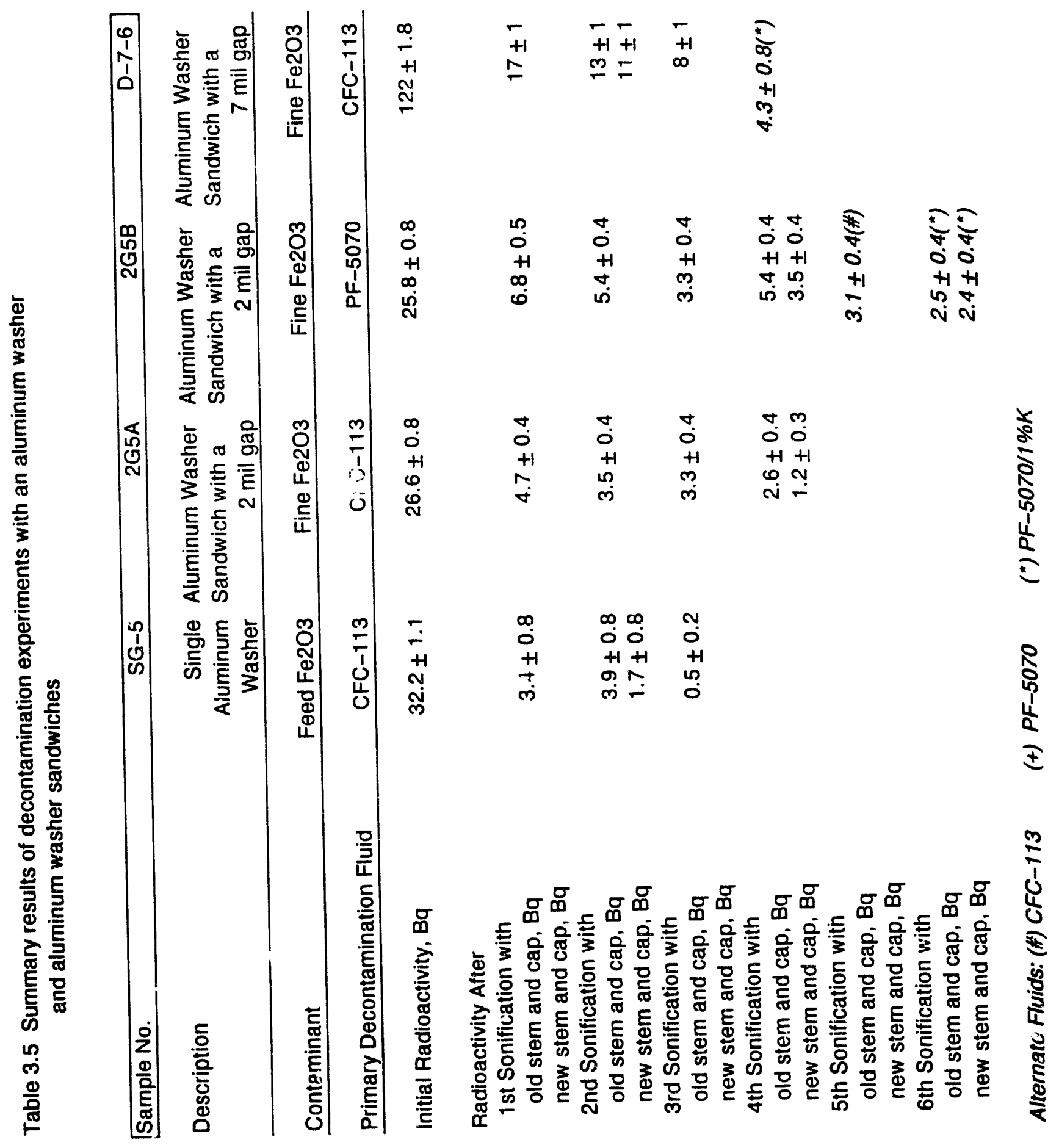




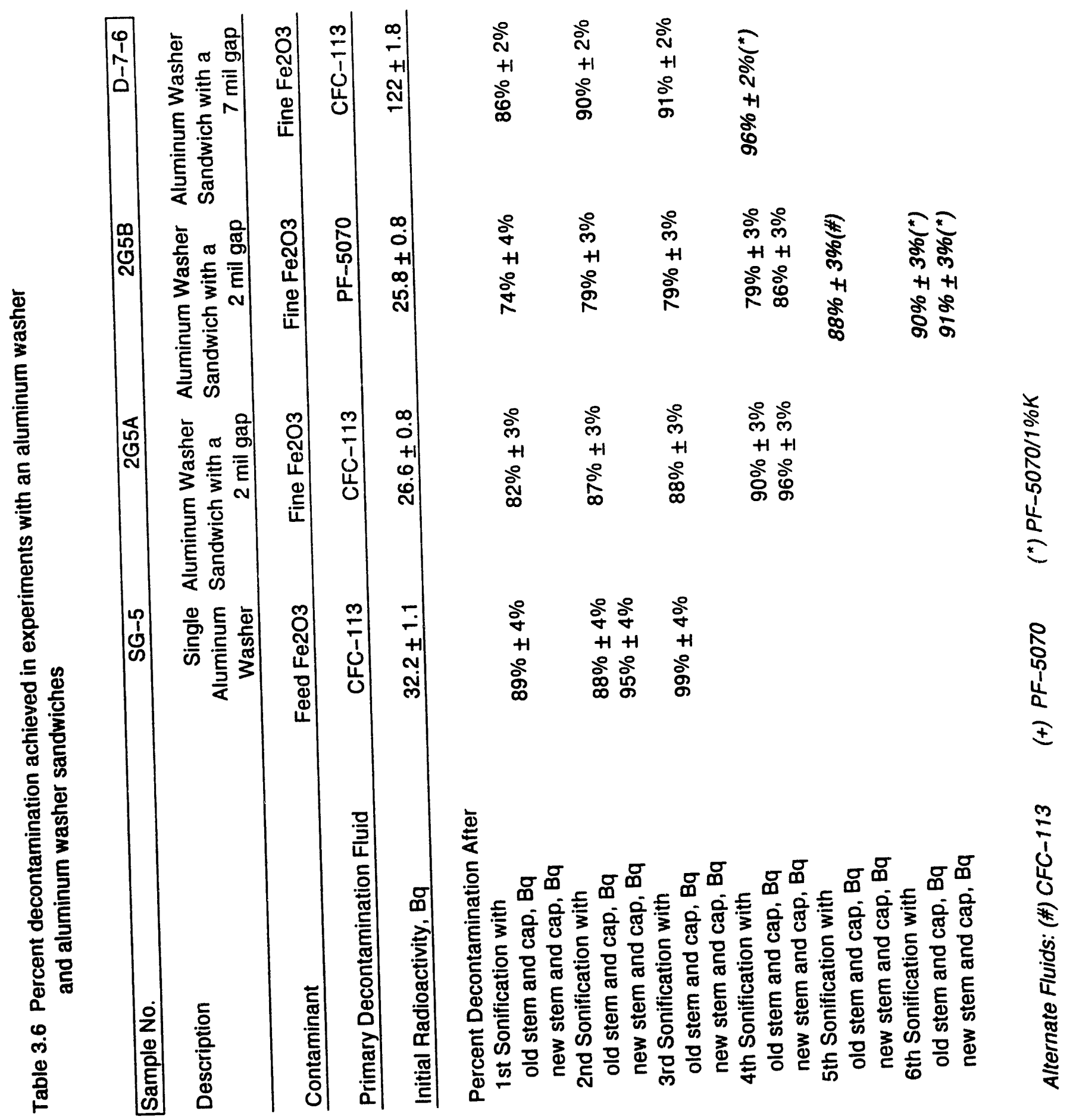




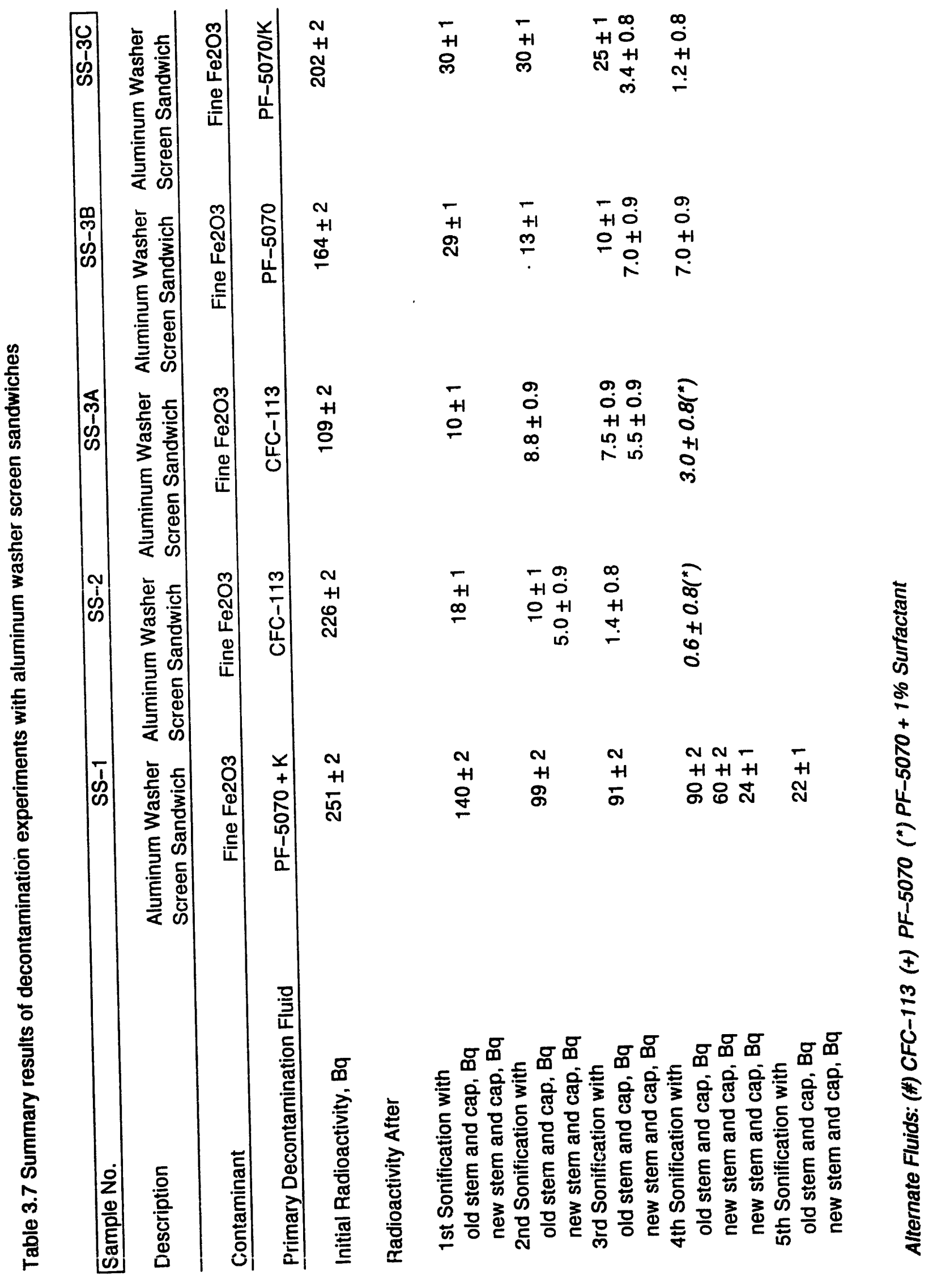




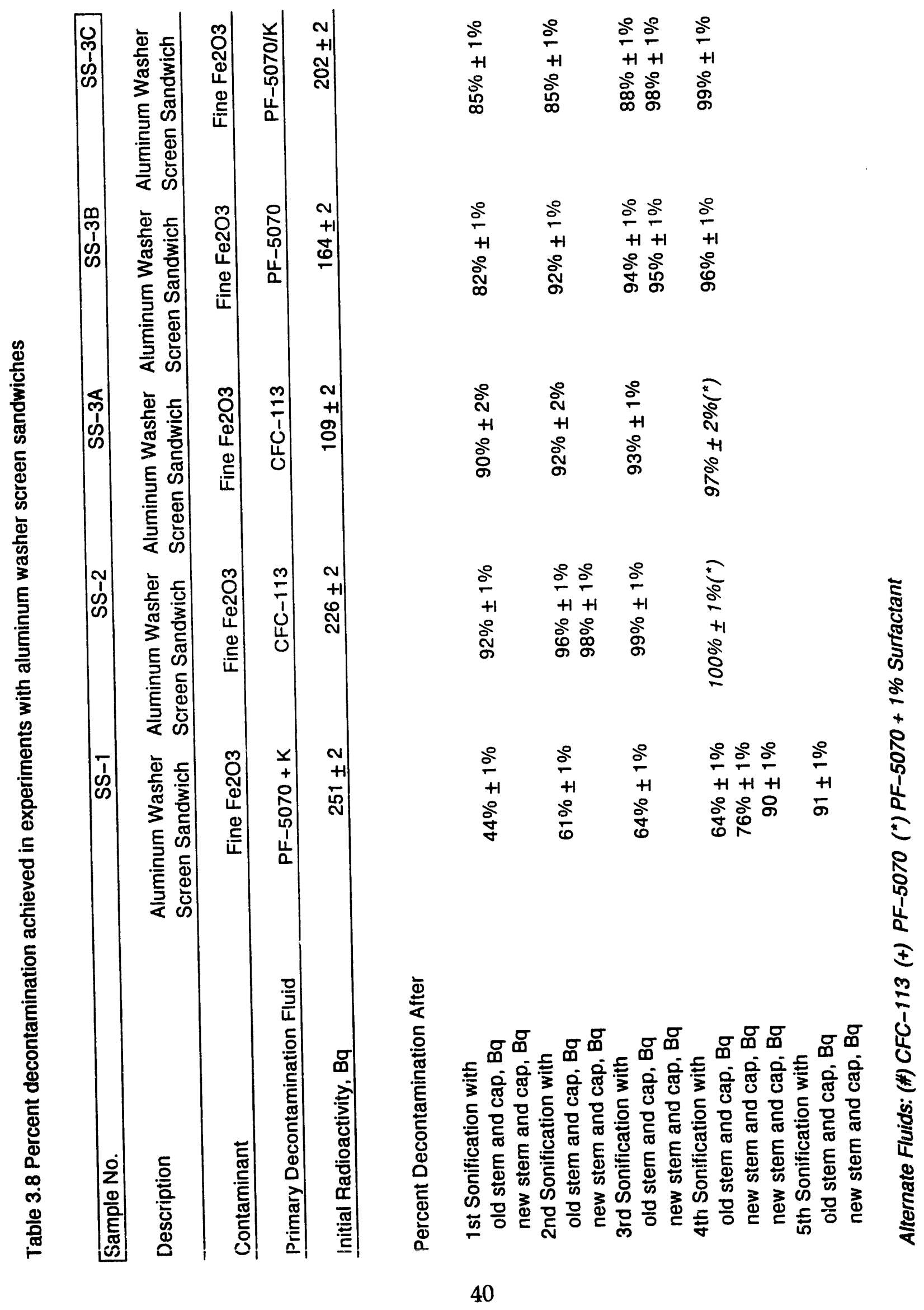




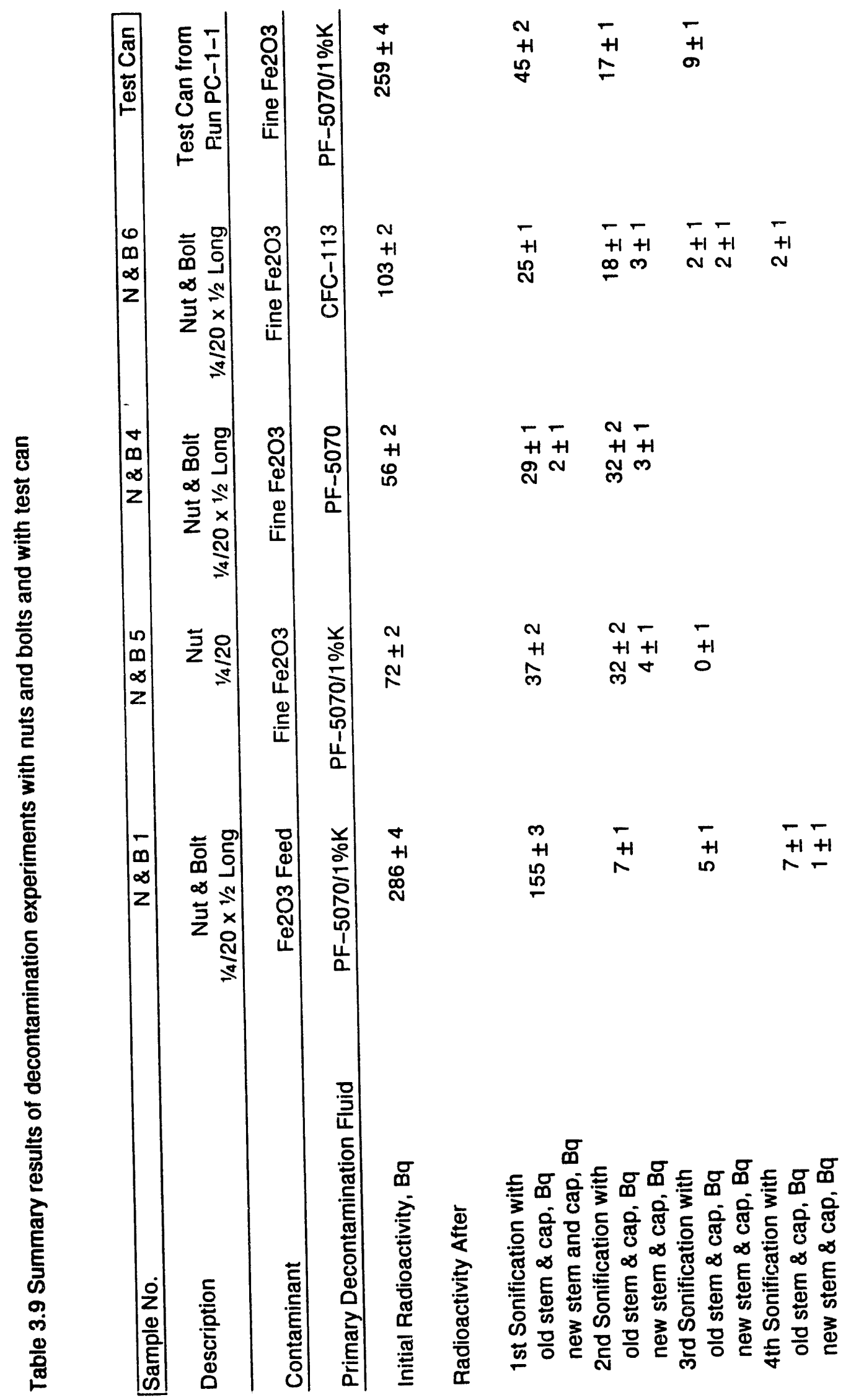




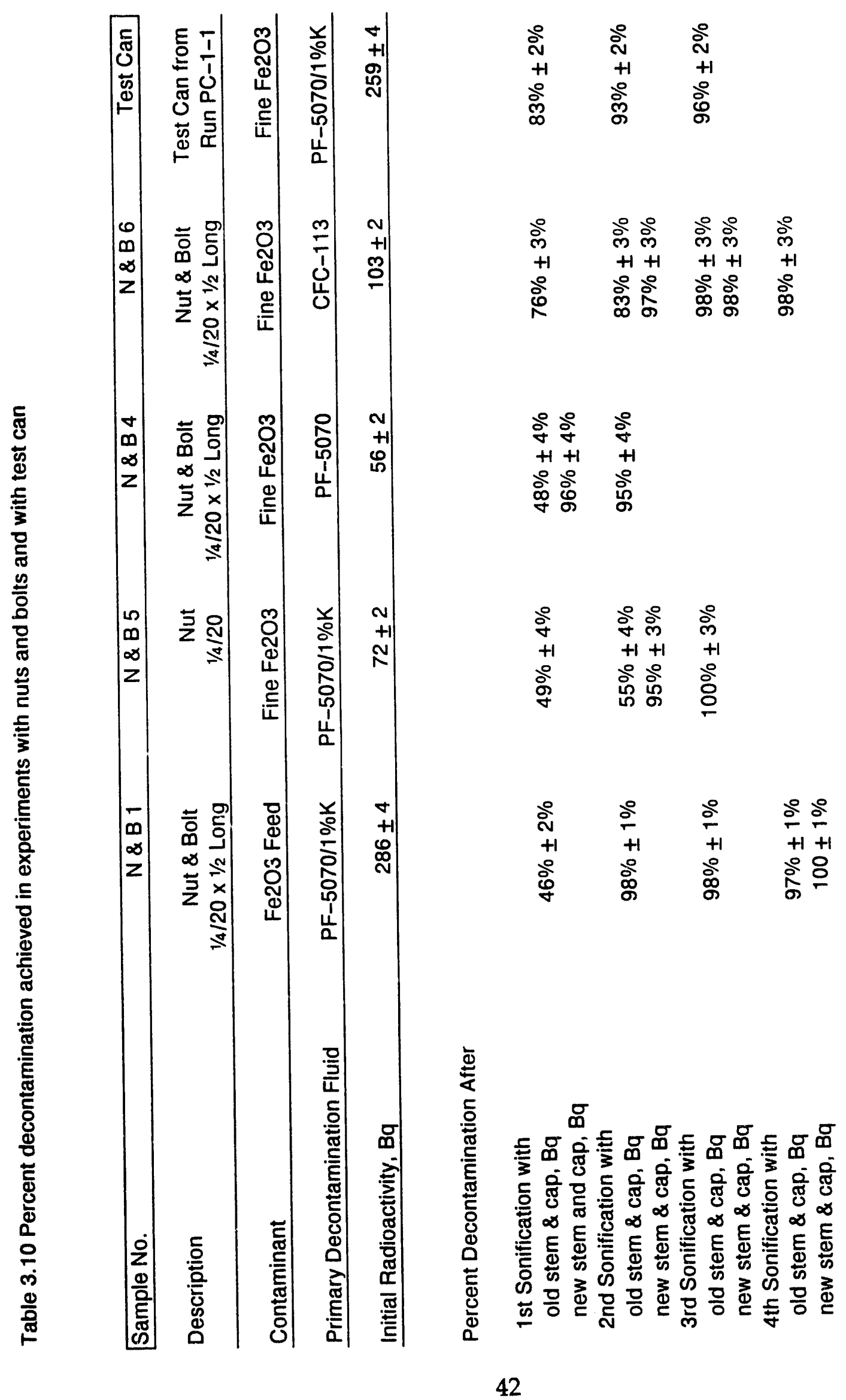


Table 3.11 Summary results of decontamination experiments with a printed circuit board

"club sandwich"

Sample No.

Description

Contaminant
PC-1

$\mathrm{PC}$ Board "Club Sandwich" with 2 mil gaps

Fine $\mathrm{Fe} 2 \mathrm{O} 3$
Process Step
Decontamination

Liquid
Radioactivity

$\mathrm{Bq}$
Decontamination

Achieved

\section{Contaminated Sample}

After 1 st Sonification with old stem and cap new stem and cap

After 2nd Sonification with old stem and cap new stem and cap

After 3rd Sonification with old stem and cap new stem and cap

After 4th Sonification with old stem and cap new stem and cap

After 5th Sonification with old stem and cap new stem and cap

After 6th Sonification with old stem and cap new stem and cap

After 7th Sonification with old stem and cap new stem and cap

After 8th Sonification with old stem and cap new stem and cap

$$
322 \pm 4
$$

$0 \%$

$$
\text { CFC-113 }
$$

$$
91 \pm 2
$$

$72 \% \pm 2 \%$

$$
\text { CFC-113 }
$$

$75 \pm 2$

$77 \% \pm 1 \%$

CFC-113

$70 \pm 2$

$78 \% \pm 1 \%$

CFC-113

$64 \pm 2$

$80 \% \pm 1 \%$

PF-5070

$54 \pm 2$

$83 \% \pm 1 \%$

PF-5070/1\%K

$46 \pm 2$

$86 \% \pm 1 \%$

PF-5070/1\%K

$48 \pm 2$

$85 \% \pm 1 \%$

PF-5070/1\%K

PF-5070/1\%K

$43 \pm 2$

$38 \pm 2$

$86 \% \pm 1 \%$

$88 \% \pm 1 \%$ 
Table 3.12 Results summary of decontamination studies

\begin{tabular}{|c|c|c|c|c|c|c|}
\hline \multirow[t]{2}{*}{ Sample } & \multirow{2}{*}{\multicolumn{2}{|c|}{$\begin{array}{c}\text { Sonication } \\
\text { Cycles }\end{array}$}} & \multirow{2}{*}{$\begin{array}{r}\text { Process } \\
\text { Liquid }\end{array}$} & \multicolumn{2}{|c|}{ Contamination Level, Bq } & \multirow{2}{*}{$\begin{array}{l}\text { Decontamination } \\
\text { Achieved, } \%\end{array}$} \\
\hline & & & & Initial & Final & \\
\hline SG-5 & & 3 & $C F$ & 32.2 & 1 & $99 \%$ \\
\hline 2G5A & & 4 & CF & 25.6 & 1 & $96 \%$ \\
\hline $2 \mathrm{G} 5 \mathrm{~B}$ & & 6 & $P F, C F, K$ & 25.8 & 2 & $91 \%$ \\
\hline$D-7-6$ & & 4 & $\mathrm{C}, \mathrm{K}$ & 122 & 4 & $96 \%$ \\
\hline SS-1 & & 5 & K & 251 & 22 & $91 \%$ \\
\hline SS-2 & & 4 & $\mathrm{CF}$ & 226 & 1 & $100 \%$ \\
\hline SS-3A & & 4 & $\mathrm{CF}$ & 109 & 3 & $97 \%$ \\
\hline SS-3B & & 4 & PF & 164 & 7 & $96 \%$ \\
\hline$S S-3 C$ & & 4 & $\mathrm{~K}$ & 202 & 1 & $99 \%$ \\
\hline N\&B1 & & 4 & $\mathrm{~K}$ & 286 & 1 & $100 \%$ \\
\hline N\&B5 & & 3 & $\mathrm{~K}$ & 72 & 0 & $100 \%$ \\
\hline N\&B4 & & 2 & PF & 56 & 3 & $95 \%$ \\
\hline N\&B6 & & 4 & CF & 103 & 2 & $98 \%$ \\
\hline Test Can & & 3 & $\mathrm{~K}$ & 259 & 9 & $96 \%$ \\
\hline$P C-1$ & & 8 & $C F, P F, K$ & 322 & 38 & $88 \%$ \\
\hline \multicolumn{3}{|c|}{ Process Liquid: } & \multicolumn{3}{|c|}{$\begin{array}{l}C F=C F C-113 \\
P F=P F-5070 \\
K=1 \% \text { Krytox } 157 F S(M) \text { in PF-5070 }\end{array}$} & . \\
\hline \multicolumn{3}{|c|}{ Contaminant: } & \multicolumn{3}{|c|}{$\begin{array}{l}\text { Feed Material: Runs SG-5 and N\&B } 5 \\
\text { Fine Material: All other runs }\end{array}$} & \\
\hline
\end{tabular}


the decontamination experiments performed with a printed circuit board "club sandwich".

The uncertainty associated with the data presented in these tables corresponds to the calculated statistical error, one standard deviation, in the radiation measurements. Because these measurements follow Poisson statistics, this error is equal to the square root of the radiation count obtained.

\subsection{Discussion of Results}

\subsubsection{Effectiveness of Decontamination Solutions}

The overall results of the decontamination tests presented in Tables 3.5 to 3.11 are summarized in Table 3.12. As can be seen from this table, contamination levels were reduced by one to better than two orders of magnitude by ultrasonic cleaning in CFC-113 or perfluorinated liquids. Contamination levels were reduced by $80 \%$ to $100 \%$ by ultrasonic cleaning in CFC- 113 , by $86 \%$ to $96 \%$ by ultrasonic cleaning in PF-5070, and by $91 \%$ to $100 \%$ by ultrasonic cleaning in a $1 \%$ solution of Krytox 157FS(M) in PF-5070. These results indicate that the perfluorinated liquids used in the study are, at least, comparable to CFC-113 as decontamination media. While there are indications that the fluorinated surfactant solution was more effective than CFC-113 and PF-5070, because of the high decontamination levels attained, and the statistical uncertainties associated with distinguishing between small numbers, the results obtained are not conclusive evidence in this regard.

\subsubsection{Decontamination criteria}

10 CFR Part 20, which establishes standards for protection against radiation, does not specify limits for surface contamination. Each applicant for a license may propose and justify what removable surface contamination limits will be allowable before decontamination will be performed. These limits should be based on the need to avoid transfer of significant amounts of contamination to unrestricted areas, and to maintain exposures as low as is reasonably achievable.

While the residual level of contamination below which parts are no longer considered to be radioactive is not specified by statute, the NRC has published guidelines which establish limits of removable and fixed radioactive surface contamination that can be present on surfaces of floors, walls, furnishings, and equipment. Maximum allowable radiation limits for surface contamination specified by Regulatory Guide 8.23 (U.S. Nuclear Regulatory Commission, 1979) are presented in Table 3.13 .

Maximum allowable radiation limits depend on the type of emitter, whether the contamination is considered removable or fixed, and whether the surface is in a 


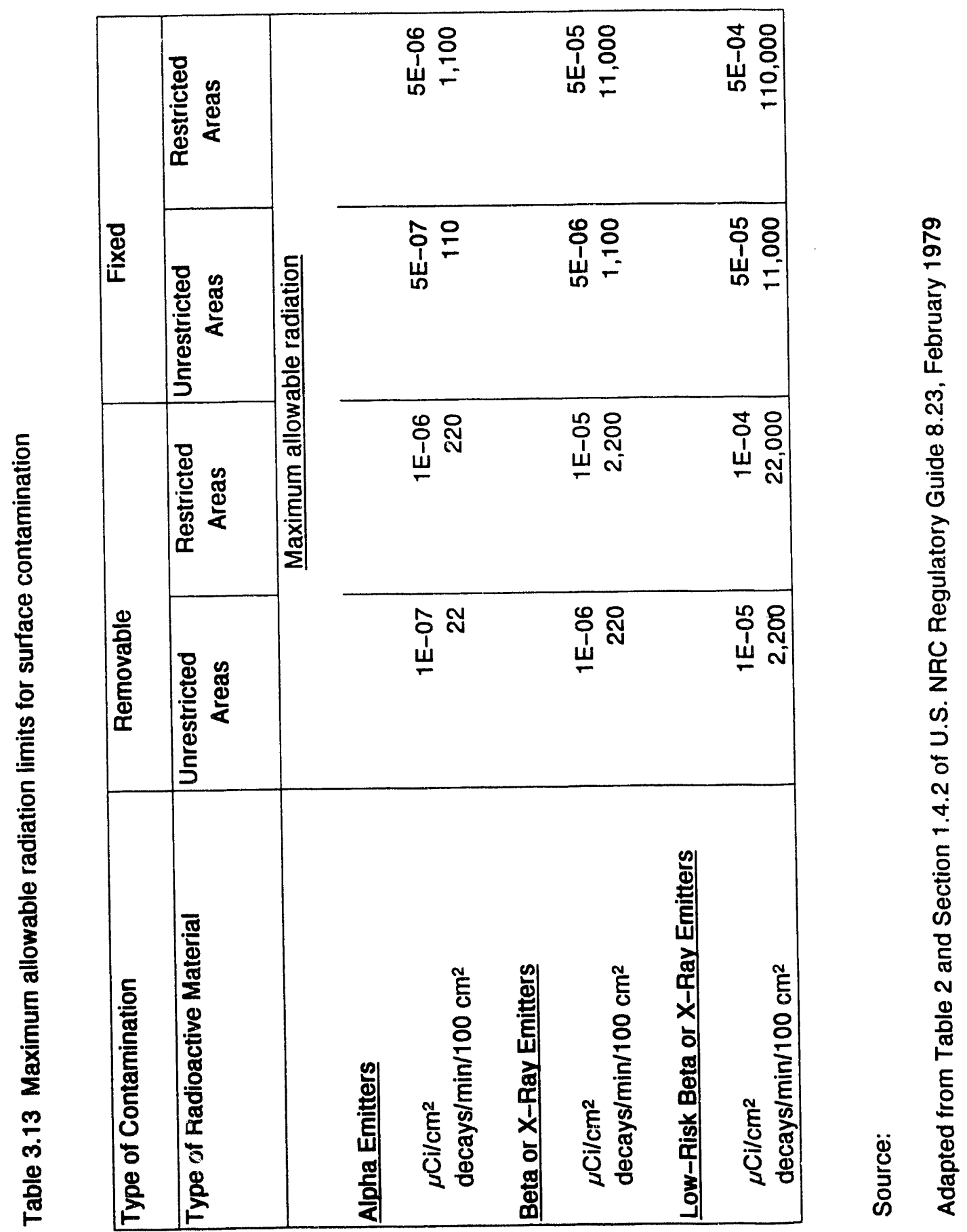


restricted or unrestricted area.

Three types of emitters are considered: alpha, beta or $x$-ray, and low-risk beta or $x$-ray. Low risk nuclides are defined as those with maximum beta energies of less than $0.2 \mathrm{MeV}$, gamma or $\mathrm{x}$-ray emission of less than $0.1 \mathrm{R} / \mathrm{h}$ at 1 meter per curie, and with a permissible concentration in air greater than $10^{\wedge}-6 \mu \mathrm{Ci} / \mathrm{ml}$. Strictest limits are placed on alpha emitters. Allowable levels are ten times higher for beta and $x$-ray emitters than for alpha emitters, and in turn, ten times higher again for low risk beta and $x$-ray emitters.

Removable contamination is defined as radioactivity that can be removed from a surface to a smear test paper by rubbing with moderate pressure. Fixed contamination is defined as radioactivity remaining on a surface after repeated decontamination attempts fail to significantly reduce the contamination level. Allowable radiation limits are five times higher for fixed contamination than for removable contamination.

Allowable levels for unrestricted areas are ten times smaller than the levels allowed in a restricted area.

Depending on the conditions, allowable radiation limits can range from 22 decays $/$ minute $/ 100 \mathrm{~cm}^{\wedge} 2$ for unrestricted release of equipment contaminated with removable alpha emitters, to 110,000 decays/minute $/ 100 \mathrm{~cm}^{\wedge} 2$ for equipment contaminated with fixed low risk beta or $x$-ray emitters that would be used in a restricted area.

Residual contamination levels attained with the various parts examined in the study are presented in Table 3.14. In this table, the residual levels were calculated on the basis of the total surface area of the components of the part assembly.

As indicated in this table, within a statistical error of one standard deviation, residual contamination levels ranged from background to 6,500 decays / 100 $\mathrm{cm}^{\wedge} 2 /$ minute for Part SS-1. Residual contamination levels of 2,200 decays / 100 $\mathrm{cm}^{\wedge} 2 /$ minute of less were attained for thirteen of the fifteen parts examined. This decontamination level was not attained with Parts SS-1 and PC-1.

The number of parts that were decontaminated to a level sufficient to allow their unrestricted release depends on whether the residual contamination is considered removable or fixed, and whether the $\mathrm{Fe}(59)$ used in the study is considered to be a standard-risk or low-risk emitter, as indicated in Table 3.15. Depending on the criterion used, the number of the fifteen test samples examined that were successfully decontaminated ranged from five to fifteen.

The iron oxide powders used in the study meets the criteria for a low risk beta or $x$-ray emitter based on the data presented in Table 3.3. 
Table 3.14 Decontamination levels attained with parts examined in study

\begin{tabular}{|c|c|c|c|c|c|c|c|}
\hline Sample & $\begin{array}{c}\text { Sonication } \\
\text { Cycles }\end{array}$ & $\begin{array}{r}\text { Process } \\
\text { Liquid }\end{array}$ & \multicolumn{2}{|c|}{$\begin{array}{c}\text { Final (Residual) } \\
\text { Contamination } \\
\mathrm{Bq}\end{array}$} & $\begin{array}{c}\text { Total } \\
\text { Surface } \\
\text { Area, } \mathrm{cm}^{2} \\
\end{array}$ & \multicolumn{2}{|c|}{$\begin{array}{l}\text { Decays } / \text { minute } \\
\text { per } 100 \mathrm{~cm}^{2} \text { of } \\
\text { Total Area }\end{array}$} \\
\hline SG-5 & 3 & $C F$ & $0.5 \pm$ & 0.2 & 10 & $296 \pm$ & 118 \\
\hline 2G5A & 4 & CF & $1.2 \pm$ & 0.3 & 20 & $355 \pm$ & 89 \\
\hline 2G5B & 6 & $P F, C F, K$ & $2.4 \pm$ & 0.4 & 20 & $710 \pm$ & 118 \\
\hline$D-7-6$ & 4 & C, $K$ & $4.3 \pm$ & 0.8 & 20 & $1,273 \pm$ & 237 \\
\hline SS-1 & 5 & $k$ & $22 \pm$ & 1.0 & 20 & $6,513 \pm$ & 296 \\
\hline SS-2 & 4 & $C F$ & $0.6 \pm$ & 0.8 & 20 & $178 \pm$ & 237 \\
\hline SS-3A & 4 & CF & $3 \pm$ & 0.8 & 20 & $888 \pm$ & 237 \\
\hline SS-3B & 4 & PF & $7 \pm$ & 0.9 & 20 & $2,072 \pm$ & 266 \\
\hline SS-3C & 4 & $K$ & $1.2 \pm$ & 0.8 & 20 & $355 \pm$ & 237 \\
\hline N\&B1 & 4 & K & $1 \pm$ & 1 & 15 & $400 \pm$ & 400 \\
\hline N\&B5 & 3 & $\mathrm{~K}$ & $0 \pm$ & 1 & 5 & $0 \pm$ & 1,200 \\
\hline N\&B4 & 2 & PF & $3 \pm$ & 1 & 15 & $1,200 \pm$ & 400 \\
\hline N\&B6 & 4 & CF & $2 \pm$ & 1 & 15 & $800 \pm$ & 400 \\
\hline Test Can & 3 & $K$ & $9 \pm$ & 1 & 164 & $329 \pm$ & 37 \\
\hline$P C-1$ & 8 & $C F, P F, K$ & $38 \pm$ & 2 & 39 & $5,890 \pm$ & 310 \\
\hline \multirow{3}{*}{\multicolumn{2}{|c|}{ Process Liquid: }} & \multicolumn{6}{|c|}{$C F=C F C-113$} \\
\hline & & \multicolumn{6}{|c|}{$P F=P F-5070$} \\
\hline & & \multicolumn{6}{|c|}{$K=1 \%$ Krytox $157 F S(M)$ in PF -5070} \\
\hline \multicolumn{2}{|c|}{ Contaminant: } & \multicolumn{6}{|c|}{$\begin{array}{l}\text { Feed Material: Runs SG-5 and N\&B } 5 \\
\text { Fine Material: All other runs }\end{array}$} \\
\hline
\end{tabular}




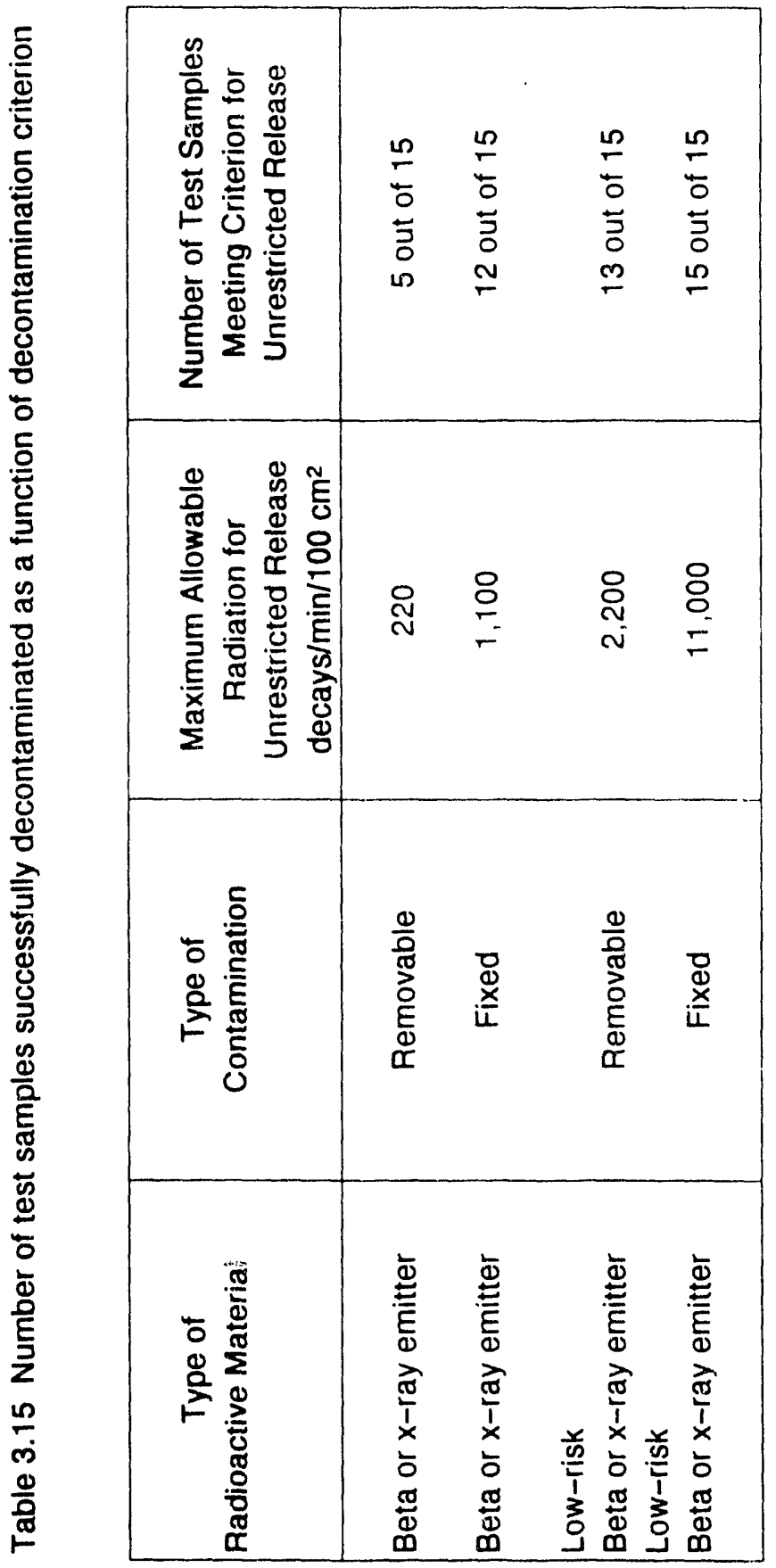


It can thus be argued that the residual contamination on the test samples used in this study was fixed contamination of a low risk beta or $x$-ray emitter, and therefore that all fifteen test samples were successfully decontaminated. The definition of fixed radiation is the radiation remaining after multiple attempts at decontamination. This criterion applies to all the test samples which were subject to a minimum of thre' sonication cycles. Furthermore, it is unlikely that rubbing the parts with sniear test paper with moderate pressure would remove the micron and sub-micron $s i z: d$ particles used in the tests which, as discussed in the introutuction, like to remain attached to whatever substrate they are on. As will be discussed further below, no visible particles were found on the external surfaces of the printed circu $:$ board pieces used in test sample PC-1, one of the samples with the highes' esidual level of contamination, when these pieces were examined under a microscope at 100x magnification after the decontamination tests. In this case, the residual radioactive particles $v$ ire in areas inaccessible to a smear wipe.

A more conservative approach would $h$ to apply a limit of 1,100 decays/minute / 100 $\mathrm{cm}^{\wedge} 2$, the criterion for fixed contamination for standard-risk beta or $x$-ray emitters. With this criterion as a basis, twelve of the fifteen samples examined could be considered releasable to uncontrolled areas.

\subsubsection{Effect of Sample Size}

Since the decontamination criterion is normalized to surface area, the use of small ar ea test pieces required essentially complete removal of the radioactive contaminant for the part to be considered nonradioactive. As a consequence, for some of the smaller test pieces, such as a nut, a statistical variation in measurement of $1 \mathrm{~Bq}$ is equivalent to an uncertainty of 1,000 decays/minute/ 100 $\mathrm{cm}^{\wedge} 2$. On a larger unit, such as the test can, which has an external area of 164 $\mathrm{cm}^{\wedge} 2$, a variation in measurement of $1 \mathrm{~Bq}$ is erquivalent to an uncertainty of 37 decays / minute $/ 100 \mathrm{~cm}^{\wedge} 2$. A residual contamination level of $7 \mathrm{~Bq}$, which corresponds to $329 \pm 37$ decays/minute $/ 100 \mathrm{~cm}^{\wedge} 2$, which is a releasable level, was attained after only three sonications.

\subsubsection{Rate of Decontamination}

Prior data $\mathrm{c} n$ the removal of nonradioactive particles that were obtained by optical means indicated that most of these particles were removed during the initial stages of the cleaning process. Representative data are presented in Figure 3.7. The number of particles removed from a suspended nut and bolt by sonication for 5 minutes in $150 \mathrm{r}$. 1 of fluorinated liquid as a function of sonication cycles is presented in this figure for different cleaning liquids of interest. These results indicate that most of the particles are removed during the first sonication cycle for all liquids. They also indicate that the significantly more particles are removed by a fluorinated surfactant solution 


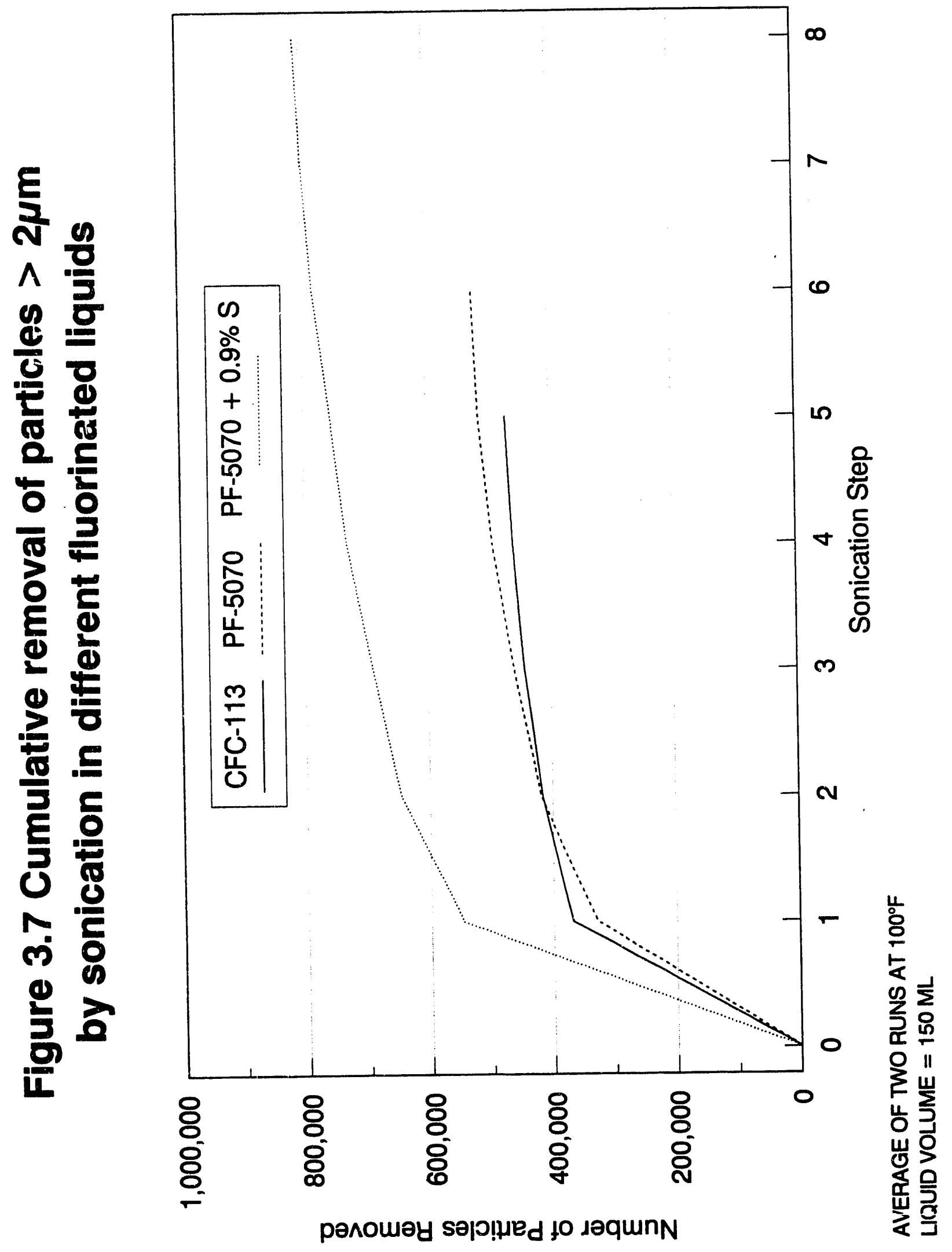


than by either PF-5070 or CFC-113, which have comparable particle removal characteristics.

The data presented in Tables 3.5 to 3.11 indicate that most of the radioactive particles are also removed during the first sonification cycle. There was, however, quite a bit of scatter in the data. This was due to spattering of decontamination liquid containing suspended radioactive material onto the stem and cap which supported the test sample during a test cycle. If the test piece is suspended from the same stem and cap in the scintillation counter after a decontamination cycle as was used to suspend it during the decontamination cycle, the apparent radioactivity of the test piece is increased by the presence of radioactive material on the stem and cap. Using the same support for multiple cleaning cycles results in an apparent rate and extent of decontamination for the test piece that are lower than the actual values because of the presence of radioactive particles on the stem and cap that are above the liquid level in the test container. Because there is no liquid contact, and therefore no transfer mechanism, any radioactive active particles deposited above the liquid line by spattering in a prior cleaning cycle are unlikely to be removed in a subsequent cycle.

The decontamination data presented in Tables 3.5 to 3.12 distinguish between the radioactivity measurements obtained with the old stem and cap (i.e. the one used during the prior cleaning cycle) and those obtained after the test piece was transferred to a new stem and cap.

The importance of this artifact, which was not accounted for in the original test plan, on the experimental results is evident from examination of the data in Tables 3.5 to 3.12. Not changing the supporting cap and stem after every cycle detracts mainly from the quality of the data on the rate of decontamination, which fortunately appears to be quite rapid, it has little impact on the ultimate level of contamination noted once the original cap and stem (i.e. the one used during the first decontamination cycle) was replaced.

With hindsight, the original experimental plan should have provided for test pieces that could be easily transferred from one supporting cap and stem to another so as to eliminate transfer of spattered particles from one decontamination cycle to the next, as was done with the final experiments performed with nuts and bolts supported on a wire loop. This, however, did not affect the final decontamination factor which was achieved.

\subsubsection{Effect of Part Structure}

As expected, geometrical complexity of the part has an effect on the ease of decontamination. High decontamination levels were obtained not only with simple parts, such as a single washer and suspended nuts and bolts, whose surfaces were exposed without restriction to the sonicating liquid, but also with the washer 
sandwiches that had gaps 0.002 in $(0.005 \mathrm{~cm})$ and 0.007 in $(0.018 \mathrm{~cm})$ wide. Because such relatively high decontamination levels were obtained with washer sandwich arrangements 2 G5 and D-7-6, screen washer sandwiches were conceived as representing a more difficult challenge since these would have interstices smaller than one tenth of the screen wire diameter, of the order of 1 mil $(0.002$ $\mathrm{cm})$. Except for sample SS-1, which had a residual radiation level of $22 \mathrm{~Bq}$ for which a decontamination level of only $91 \%$ was attained, very high decontamination ratios were obtained with this configuration. The variation between screen sand wiches is believed to be due to differences in the tightening of the screw holding the assembly together, resulting in variations in compression of the screen, and, thus, the effective spacing between the screen wires, resulting.in the trapping of radioactive particles.

The printed circuit "club sandwich" proved to be the most difficult part to decontaminate. This part had the highest residual background of all the parts that were examined. This part was closely reexamined a week after it had been originally contaminated and decontaminated. It was dismantled into its component parts which were then individually examined.

The radioactivity of the sum of the parts, as well as the individual parts, was measured, as indicated in Table 3.16. The sum of the counts obtained when the parts were measured individually was $1799 \pm 59$ counts / 100 sec versus $1537 \pm 43$ counts $/ 100 \mathrm{sec}$. This discrepancy is due to variations in the placement of the parts in the counting chamber which affects the counting efficiency.

Most of the residual radioactivity is distributed fairly evenly on the three pieces of circuit board. The radioactivity of the middle board was not significantly different than that of the outer boards.

The three pieces of circuit board were also examined microscopically, at a magnification of $100 \mathrm{X}$ with reflected light, under both light field and dark field illumination. At this magnification, it would be possible to detect the presence of micron sized particles, especially under dark field. The inspection performed indicated that the surfaces of these pieces were clean, no particles could be observed. The inspection also revealed that the small holes in the boards were drilled, and that the free ends of the glass fiber reinforcements were matted and tangled.

The relatively low decontamination of the PC board sample is ascribed to entrapment of small radioactive particles in these tangles. This hypothesis is consistent with the finding that there was no observable particles on the surfaces of the boards, and that the radioactivity of all three boards were approximately equal. These findings, supported by the high decontamination levels found for the $2 \mathrm{mil}$ gap and $7 \mathrm{mil}$ gap aluminum washer sandwiches, also indicate that a 2 mil spacing between surfaces does not represent a significant restriction to particie removal under ultrasonic agitation. 
Table 3.16 Post-cleaning examination of components of PC board test assembly

\begin{tabular}{|c|c|c|c|c|}
\hline \multirow[t]{2}{*}{ Items } & \multirow{2}{*}{$\begin{array}{l}\text { Sample Position } \\
\text { in Detector }\end{array}$} & \multicolumn{3}{|c|}{ Instrument Counts/100 sec } \\
\hline & & Gross & Background & Net \\
\hline $\begin{array}{l}3 \text { circuit board pieces, } \\
2 \text { s/s washers, } 1 \text { steel } \\
\text { screw \& } 2 \text { steel nuts }\end{array}$ & Flat on bottom & 1693 & 156 & 1537 \\
\hline $2 \mathrm{~s} / \mathrm{s}$ washers & Flat on bottom & 179 & 156 & 23 \\
\hline Steel screw \& 2 nuts & Flat on bottom & 262 & 156 & 106 \\
\hline Circuit Board Piece 1 & Flat on bottom & 683 & 156 & 527 \\
\hline Circuit Board Piece 2 & Flat on bottom & 753 & 156 & 597 \\
\hline Circuit Board Piece 3 & Flat on bottom & 702 & 156 & 546 \\
\hline $\begin{array}{l}3 \text { circuit board pieces, } \\
1 \text { steel screw \& } 2 \text { nuts }\end{array}$ & $\begin{array}{l}\text { Standard } \\
\text { Geometry }\end{array}$ & 575 & 158 & 417 \\
\hline $\begin{array}{l}3 \text { circuit board pieces, } \\
1 \text { steel screw \& } 2 \text { nuts } \\
\text { after sonifying for } 5 \text { min } \\
\text { in PF- } 5070+1 \% \mathrm{~K}\end{array}$ & $\begin{array}{l}\text { Standard } \\
\text { Geometry }\end{array}$ & 565 & 158 & 407 \\
\hline
\end{tabular}


The above test conditions are believed to represent a greater decontamination challenge than those likely to exist in the "real world". Parts do not normally become contaminated as a result of being immersed and ultrasonically agitated in a suspension of radioactive particles. This contacting method promotes more intimate contact of the part with the radioactive particles, and the inclusion of these particles into every nook and cranny. Furthermore, the printed circuit boards used in the study differ from finished printed circuit boards used in actual electronic equipment in that the holes of the latter are usually electroplated and present a smooth metal surface. The general purpose boards obtained from the Radio Shack thus represent a much more difficult decontamination problem than would normally exist with electronic equipment found in the field.

Physical removal of small particles from such matted environments is difficult, and represents a limitation for any method of physical decontamination, including the one proposed in this report. The degree of particle removal may be increased by using more effective means of agitation. The ultrasonic power supply used in the experiments is an older model that generates sinusoidal waves. The limitations of these older units is that the ultrasonic power output varied with processing conditions, and more important, was not uniform within the bath volume, resulting in so called "hot" spots and dead zones. Newer models offered by equipment suppliers, such as Crest Ultrasonics (Trenton, NJ) and the J.M. Ney Company (Bloomfield, CT), result in more uniform cavitation within the bath and lower sensitivity to process variables, resulting in much more uniform cleaning action. Approaches taken by various manufacturers include square wave generation for more efficient use of power and variable frequency generation to eliminate standing waves, the prncipal source of poor distribution of ultrasonic energy within the bath volume.

\subsubsection{Effect of Particle Size}

Two of the runs (SG-5 and N\&B-1) were carried out with the feed powder which had a weight average particle size of $11.9 \mu \mathrm{m}$ which was much coarser than the fine fraction which had a weight average particle size of $1.3 \mu \mathrm{m}$. The decontamination levels attained in runs SG-5 and N\&B-1 were both very high, with residual contamination levels approaching background in both cases. These high level of contamination are believed to be due in part to the use of a coarser contaminant, and in part due to the simple part geometries involved.

\subsubsection{Sonication of Electrical Parts}

The effect of sonication in perfluorinated liquids on the performance of resistors found in electrical circuits was evaluated to demonstrate that this treatment would not affect these components.

Standard wire wound resistors, with resistances ranging from 20 ohms to 100 
Table 3.17 Effect of sonication in perfluorinated liquids on the performance of electrical resistors

\begin{tabular}{|c|c|c|c|}
\hline Sample & Rated & Measured & istance \\
\hline & kilo-ohms & $\begin{array}{c}\text { Before } \\
\text { Treament } \\
\text { kilo-ohms }\end{array}$ & $\begin{array}{c}\text { Post } \\
\text { Treament } \\
\text { kilo-ohms }\end{array}$ \\
\hline 1 & 100 & 94.7 & 94.6 \\
\hline 2 & & 94.4 & 94.5 \\
\hline 3 & & 94.6 & 94.7 \\
\hline 4 & 1 & 0.95 & 0.95 \\
\hline 5 & & 0.95 & 0.95 \\
\hline 6 & & 0.95 & 0.95 \\
\hline 7 & 0.2 & 0.19 & 0.19 \\
\hline 8 & & 0.19 & 0.19 \\
\hline 9 & & 0.19 & 0.19 \\
\hline 10 & 0.1 & 0.1 & 0.1 \\
\hline 11 & 0.075 & 0.07 & 0.07 \\
\hline 12 & & 0.07 & 0.07 \\
\hline 13 & & 0.07 & 0.07 \\
\hline 14 & 0.02 & 0.02 & 0.02 \\
\hline 15 & & 0.02 & 0.02 \\
\hline 16 & & 0.02 & 0.02 \\
\hline Treatment: & & & \\
\hline $\begin{array}{l}\text { 1. Sonicat } \\
\text { in PF-5C } \\
\text { 2. Sonicat } \\
\text { 3. Sonicat } \\
\text { 4. Air dry. }\end{array}$ & $\begin{array}{l}\text { in } 1 \mathrm{wt}-\% \text { solutic } \\
0^{\circ} \mathrm{C} \text { ). } \\
\text { in PF-5070 at } 1 \\
\text { in PF- } 5070 \text { at } 14\end{array}$ & $\begin{array}{l}\text { purified Krytox } \\
=\left(60^{\circ} \mathrm{C}\right) \\
\left(60^{\circ} \mathrm{C}\right) .\end{array}$ & $S(M)$ \\
\hline
\end{tabular}


kilo-ohms were purchased from a local electronic supply store. After measuring their resistance with a standard multimeter, these parts were sonified for 5 minutes in $200 \mathrm{ml}$ of a $0.9 \mathrm{wt} \%$ solution of purified Krytox 157FS(M) in PF-5070 at $140^{\circ} \mathrm{F}\left(60^{\circ} \mathrm{C}\right)$ in a stainless steel beaker. These parts were then rinsed twice in pure PF-5070 under the same conditions. The resistance of these components was then remeasured.

Ultrasonic agitation in perfluorinated liquids resulted in no measurable change in the resistance of these devices, as noted in Table 3.17. Decontamination tests were not performed with these components because they had very simple geometries and smooth walls, and would have represented a trivial exercise.

\subsection{Conclusions}

Sonication of a part contaminated with radioactive particles in a fluorinated liquid is an effective method of decontamination.

This method is an effective method of removing radioactive contaminants that range in size from less than one micrometer in diameter to more than 40 micrometers in diameter.

Perfluorinated liquids are comparable to CFC-113 as decontamination media.

Perfluorinated surfactant solutions are at least as effective as perflucrinated liquids and CFC-113 as decontamination media, and may be more effective.

Decontamination occurs rapidly. Most of the radioactive particles are removed within the first minutes of sonication.

The extent of decontamination is a function of the complexity of the part being decontaminated. 


\section{FILTRATION OF PROCESS LIQUIDS}

\subsection{Introduction}

For the proposed decontamination process to be operative, it is essential to be able to recycle the process liquids for further use. This requires removal of the radioactive particles from the process streams after parts decontamination. With the commercial availability of sub-micron size rated membrane filters, filtration of the process liquids through a such a membrane filter appeared to be the simplest and most effective means of removing the radioactive particles from these liquids. The filter membrane would also act as a small volume carrier for the final disposal of the recovered radioactive particles.

\subsection{Experimental Apparatus}

Filtration experiments were performed in a $500 \mathrm{ml}$ capacity Nalgene filter holder/receiver unit (Model No. 300-4050). This unit supports a $47 \mathrm{~mm}$ membrane filter and can be used to filter liquids by either vacuum or pressure. In order to minimize fluid evaporation losses, and because of concerns about the possible introduction of radioactive material into the laboratory vacuum system (in case filtration was not effective), the process liquids were filtered under pressure with compressed air. A positive pressure differential of about 0.1 atmosphere (10 $\mathrm{kPa}$ ) was used.

Filtration of radioactive suspensions were performed with $0.1 \mu \mathrm{m}$ rated PVDF (polyvinylidene fluoride) membrane filters (Millipore Corporation's Durapore ${ }^{\mathrm{TM}}$ filter membranes). ESI has found these this filter material to be stable in, and compatible with, CFC-113 and perfluorinated liquids.

\subsection{Experimental Results}

Results of filtration experiments with contaminated CFC-113 and 1\% Krytox 157FS(M) in PF-5070 are presented in Tables 4.1 and 4.2.

\subsection{Discussion of Results}

Examination of Tables 4.1 and 4.2 indicates that filtration through a $0.1 \mu \mathrm{m}$ Durapore $^{\mathrm{TM}}$ membrane filter is a very effective method of removing radioactive particles from suspension in both CFC-113 and a $1 \%$ solution of Krytox 157FS(M) in $\mathrm{PF}-5070$. Filtration reduced the radioactivity of the liquids from more than $200 \mathrm{~Bq} / 100 \mathrm{ml}$ for the feed to zero for the filtrates in both cases to within a calculated statistical error (1 standard deviation) of less than $1 \mathrm{~Bq}$. 


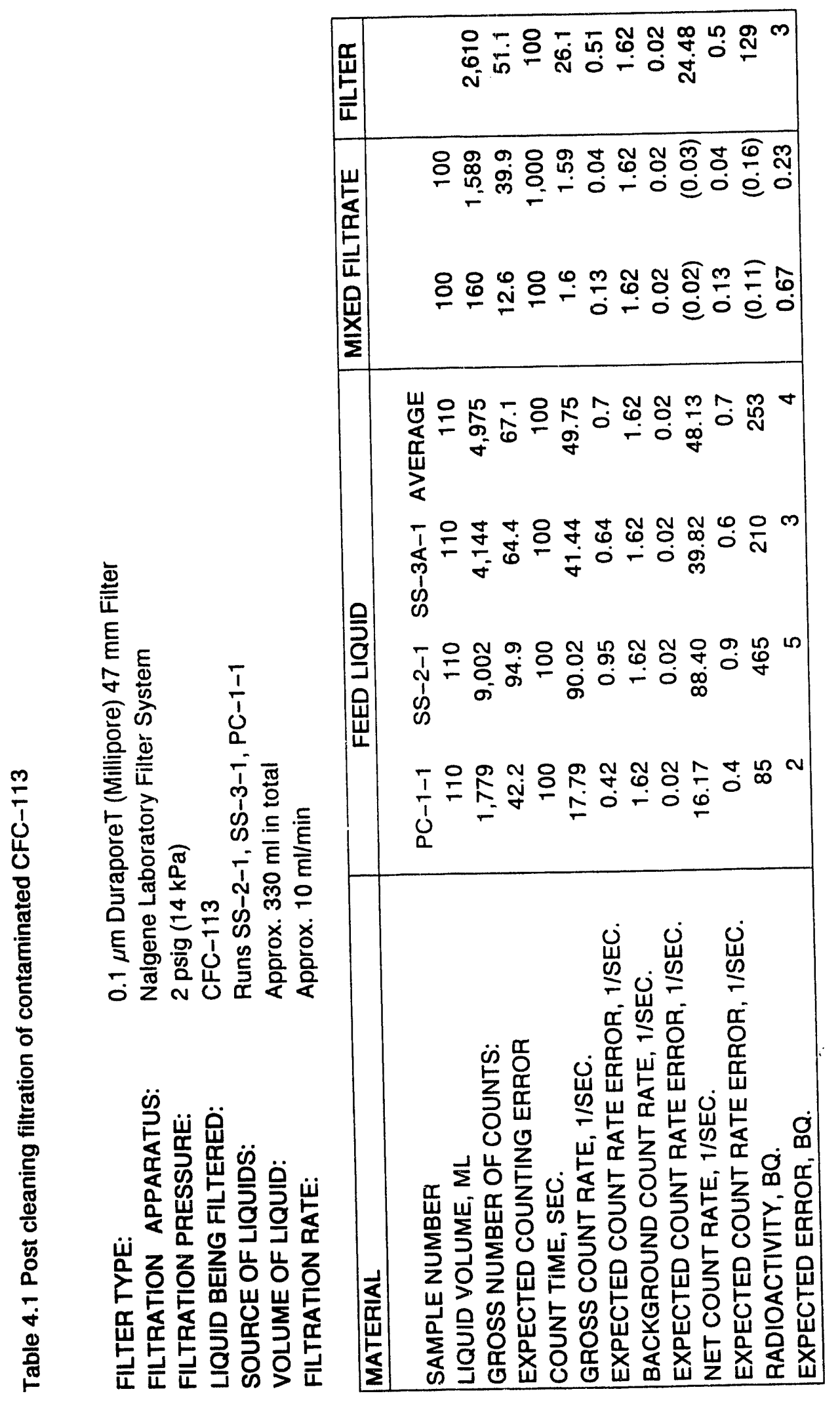




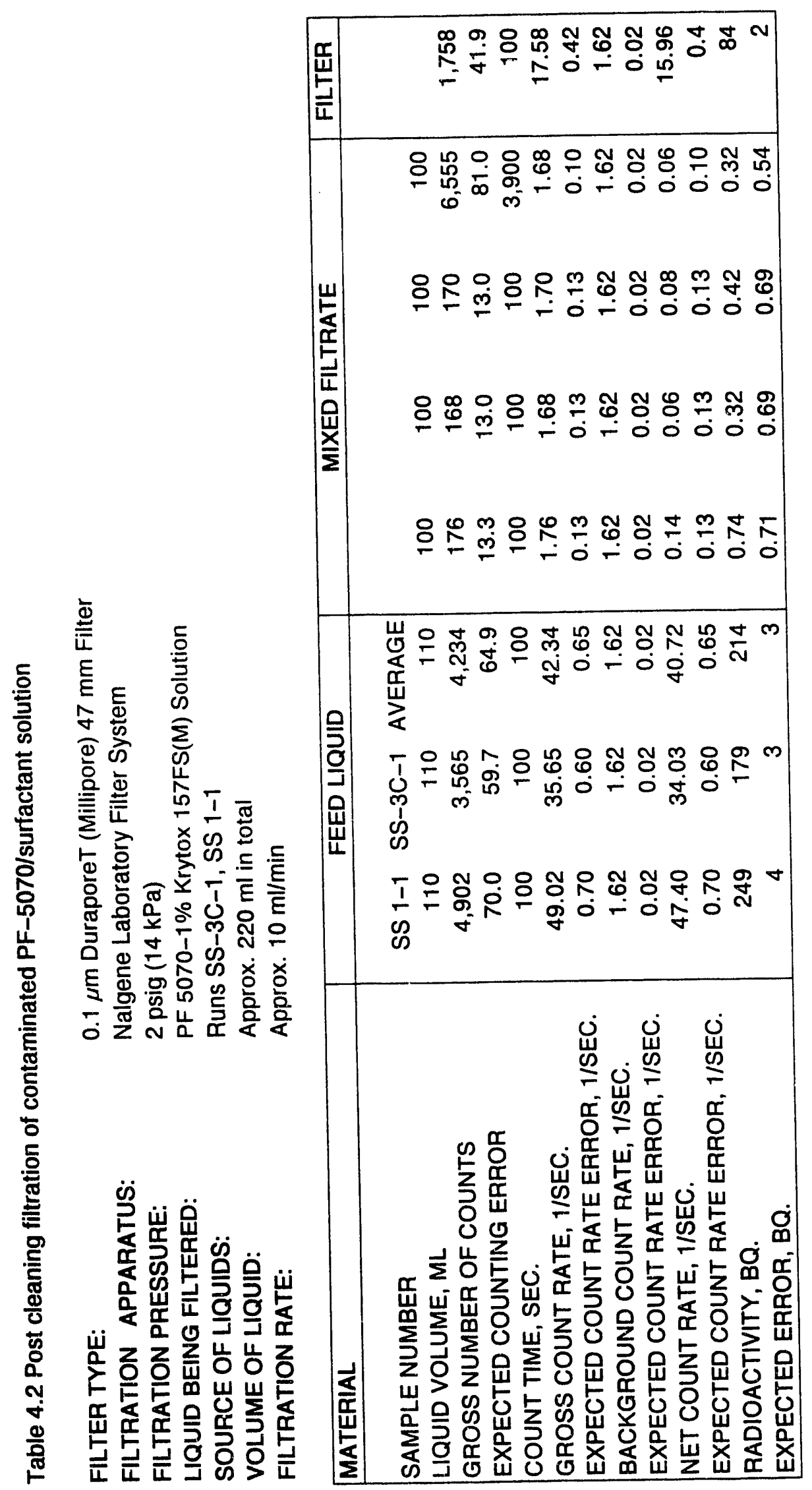


After filtration, the membrane filters were reddish brown due to the accreted iron oxide particles which formed a coherent coating on the membrane. The filters were removed from the housing with tweezers and placed in a plastic Petri dish. After measuring their radioactivity, they were set aside for disposal. The counts on the filter are smaller than the counts reported for the feed liquids by a factor of two. This difference in count is ascribed to the great differences in sample geometry.

The above are important findings in that they indicate that sub-micron sized radioactive contaminants can be removed from process liquids by membrane filtration. This allows the recycling of the process fluids and also provides a simple compact support for the disposal of the radioactive particles removed from the parts being decontaminated.

\subsection{Conclusions}

Filtration through a $0.1 \mu \mathrm{m}$ rated membrane microfilter quantitatively removes sub-micron sized radioactive particles from CFC-113 and solutions of a perfluorinated surfactant in a perfluorinated liquid.

This allows for the recycling of the process fluids and also provides a simple compact support for the disposal of the radioactive particles removed from the parts being decontaminated. 


\section{PROCESS DESCRIPTION AND ECONOMICS}

\subsection{Process Description}

The results of feasibility experiments performed during Phase I indicate that the ESI process has a high probability of being a practical and effective means of removing small, radioactive particles from contaminated parts, and that this process, once optimized, would be well suited to the needs of the nuclear industry. A conceptual flow diagram for such a process is outlined in Figure 5.1. A process description is given below.

Particle contaminated parts are loaded in an open mesh basket which is lowered into an ultrasonic bath. A vapor tight cover is then lowered over the bath to prevent vapor losses during operation. A fluorinated washing liquid is introduced into the bottom of the ultrasonic bath. The washing liquid is a dilute solution of a perfluorinated surfactant, such as purified Kry tox 157FS (Du Pont Corp.), in a perfluorinated liquid, such as perfluoroheptane (PF-5070, 3M Corp.). Washing liquid is drawn from reservoir $\mathrm{T}-1$, and pumped continuously into the ultrasonic bath, which is activated once filled with liquid. Washing liquid leaves the bath over an overflow weir, and is returned to tank T-1, through a filter, F-1, which continuously removes radioactive particles that were detached from the contaminated part and are suspended in the wash liquid. The ultrasonic bath and tank T-1 both contain heating coils to control the temperature of the recirculated wash liquid. The feed stream to the bath is filtered through filter F-2 to remove foreign particles from the wash solution entering the bath. The concentrations of particles in the stream leaving the bath, and of the concentration of perfluorinated surfactant in the washing liquid are monitored by periodic sampling. Particle concentration is obtained by measuring the radioactivity of this sample in a scintillation counter. Surfactant concentration is obtained by measuring light absorption at $210 \mathrm{~nm}$ in a ultraviolet spectrophotometer.

Because it is anticipated that the amount of time needed to remove particles from different parts will be a function of their geometry, it is proposed to use the concentration of particles in the bath effluent stream, rather than time, to monitor and control the process. Washing liquid is circulated through the ultrasonic bath until the radioactivity of the effluent stream approaches background level for a predetermined period of time. Ultrasonic agitation is stopped, wash solution is no longer circulated through the tank, and the tank is drained of holdup wash liquid.

Fluorinated rinse liquid is now introduced into the bottom of the ultrasonic bath. The rinse liquid is preferably the same material as the carrier liquid used in 


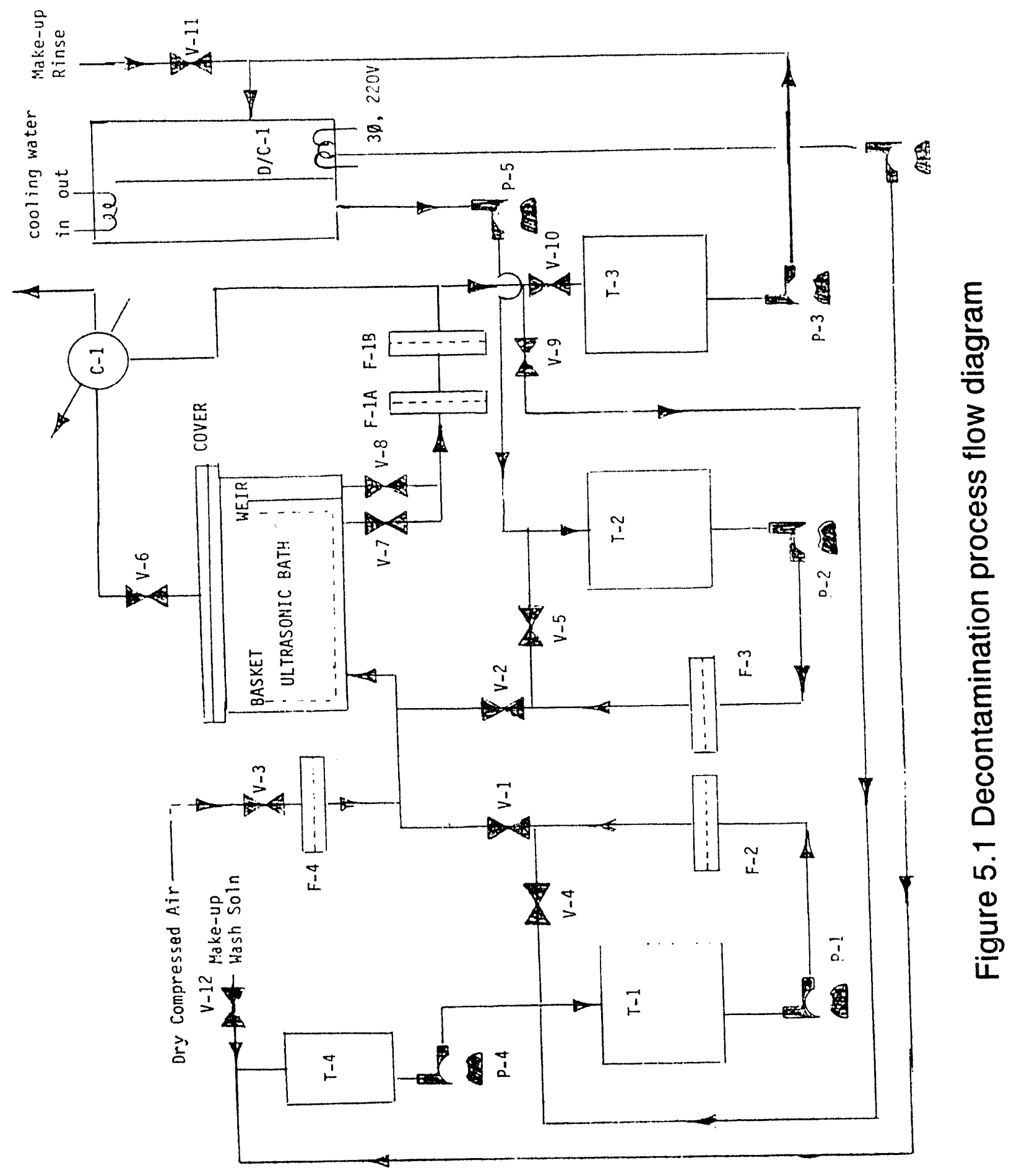


the washing solution. Rinse liquid is drawn from reservoir T-2, which can be heated to control liquid temperature, and pumped continuously into the ultrasonic bath, which is activated once filled with liquid. Rinse liquid leaves the bath over the overflow weir, and is sent to a holdup tank, $\mathrm{T}-3$. The feed stream to the bath is filtered. through filter F-3 to remove foreign particles from the incoming rinse liquia entering the bath.

Because it is anticipated that the amount of time needed to remove residual su i actant from different parts will bu a function of their geometry, it is proposed to use the concentration of s.urfactant in the bath effluent stream to monitor and control the process. Rinse liquid is circi i ied through the ultrasonic bath until the surfactant concentration in the effluent stream is below the detection limit of the monitoring instrument for a predetermined period of time. Once this level is reached, ultrasonic agitation is stopped, rinse liquid is no longer circulated through the tank, and the tank is drained of holdup rinse liquid.

Filtered, conditioned air is now introduced into the bottom of the drained ultrasonic bath to remove by evaporation residual rinse liquid wetting the cleaned parts. Because of the very low heai of evaporation of perfluorinated liquids, evaporation will be carried out adiabatically. There should be sufficient heat capacity in the cleaned parts, especially if they were cleaned at a temperature above ambient, to evaporate the residual fluorinated rinse. Once the parts are dry, the cover of the ultrasonic bath is removed, and the basket of cleaned parts is lifted out. The ultrasonic tank is now ready to accept a new basket of parts that need to be cleaned.

The vapors of evaporated fluorinated liquid can be recovered from the air stream leaving the system by a number of means. For small scale systems, the most effective means would be to chill the gas stream to a temperature such that the vapor pressure of the fluorocarbon liquid is reduced :o less than $10 \%$ of the vapor pressure at ambient. For example, reducing the temperature of an air/PF-5070 gas stream to $0^{\circ} \mathrm{F}$ (where the vapor pressure of PF-5070 is $7.4 \mathrm{~mm}$ ) would reduce the PF-5070 concentration in the effluent gas to less than $1 \%$, and would result in over 95\% recovery of the PF-5070 evaporated from the parts in the ultrasonic bath. The condensed vapors are recycled to tank 13 . For larger scale systems, vapor permeation recovery systems such as those being developed by Membrane Technology and Research, Inc., Menlo Park, CA, would becnme cost effective.

Used rinse liquid from the ultrasonic bath, which contains a small amount of fluorocarbon surfactant removed from the cleaned parts, accumulates in holdup tank T-3 before being pumped to a recovery still/ condenser, D/C.-1, Because the fiuorucarbon suiffactant is a nonvolatile oil with a vapor pressure of many orders of magnitude less than that of the rinse liquid, purification is accomplished by a simple single stage distillation-condensation process. The overhead product 
is purified rinse liquid that is returned to Tank T-2. The siill bottoms are a solution of surfactant in rinse liquid that is recycled as makeup washing solution. The surfactant concentration in the still bottoms is controlled by munitoring the boiling temperature of these bottoms; it is estimated that the boiling temperature of the bottoms increases approximately $0.5^{\circ} \mathrm{F}$ for every mole-percent increase in surfactant concentration. Alternatively, the surfactant concentration in the still bottoms could also be measured directly by UV. The still bottoms are recycled to holdup tank $\mathrm{T}-4$. This concentrated surfactant solution is then diluted to wash solution concentration by blending it with freshly distilled rinse liquid.

Rinse liquid make up is added to the still/condenser feed stream, wash solut $\mathrm{n}$ make up is added to tank T-4.

Radioactive particles removed from the parts being cleaned will accumulate in the recycled wash solution, and from which they would be removed by Filter F-1. Periodic replacement of this filter thus eliminates these particles from the process. The filter is also the waste disposal package for these radioactive particles.

\subsection{Projected Process Economics}

\subsubsection{Projected System Capital Cost}

The estimat $t$ cost of purchased equipment and process materials for a small scale prototype decontamination system with a 5 gallon ultrasonic bath capacity, based on the process description provided in section 5.2, is presented in Table 5.1. Purchïsed equipment is estimated tr be $\$ 48,000$, and process materials $\$ 9,300$, for a total projected system cost of about $\$ 57,000$. This cost does not include any charges for system design and engineering, assembly and test. Taking these charges into account, it is estimated that a prototype system can be delivered for about $\$ 100,000$.

It is projected that a commercial size system would have an ultrasonic bath would be approximately $3 \mathrm{ft}$ on a side, and have a capacity of approximately $200 \mathrm{gal}$ ( 27 $\mathrm{ft}^{\wedge} 3$ ). Such a unit would be able to handle larger parts, such as $5 \mathrm{hp}$ motors. Assuming 0.4 power scaling, it is estimated that such a system would cost approximately $\$ 400,000$.

\subsubsection{Projected System Operating Costs}

Projected costs of system operation for a smail scale unit are presented in Table 5.2. Direct operating costs are estimated to be of the order of $\$ 49$ per hour, based on the assumptions given in Table 5.2. The major operating cost element is direct labor. It was assumed that unit would have a dedicated operator in use whose burdened cost (including all indirect costs) would be $\$ 30 / \mathrm{hr}$. Capital 


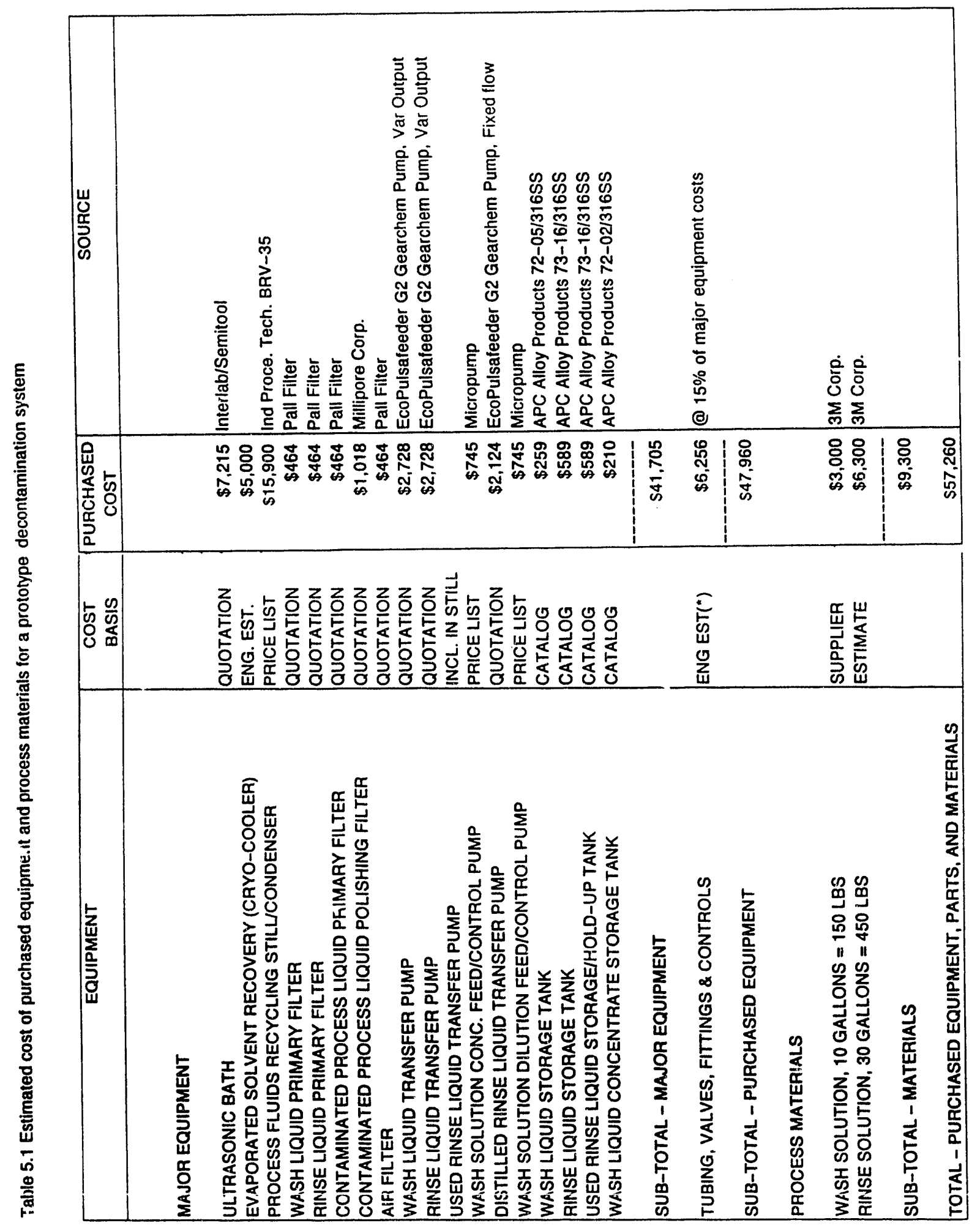


Table 5.2 Estimated projected operating costs of a small ( 5 gallon bath) ESI decontamination system

\section{Operating Assumptions:}

Operating Shifts/day: 1

Operating Hours/day: 8

Operating Days/year: 200

System Life, yrs: 5

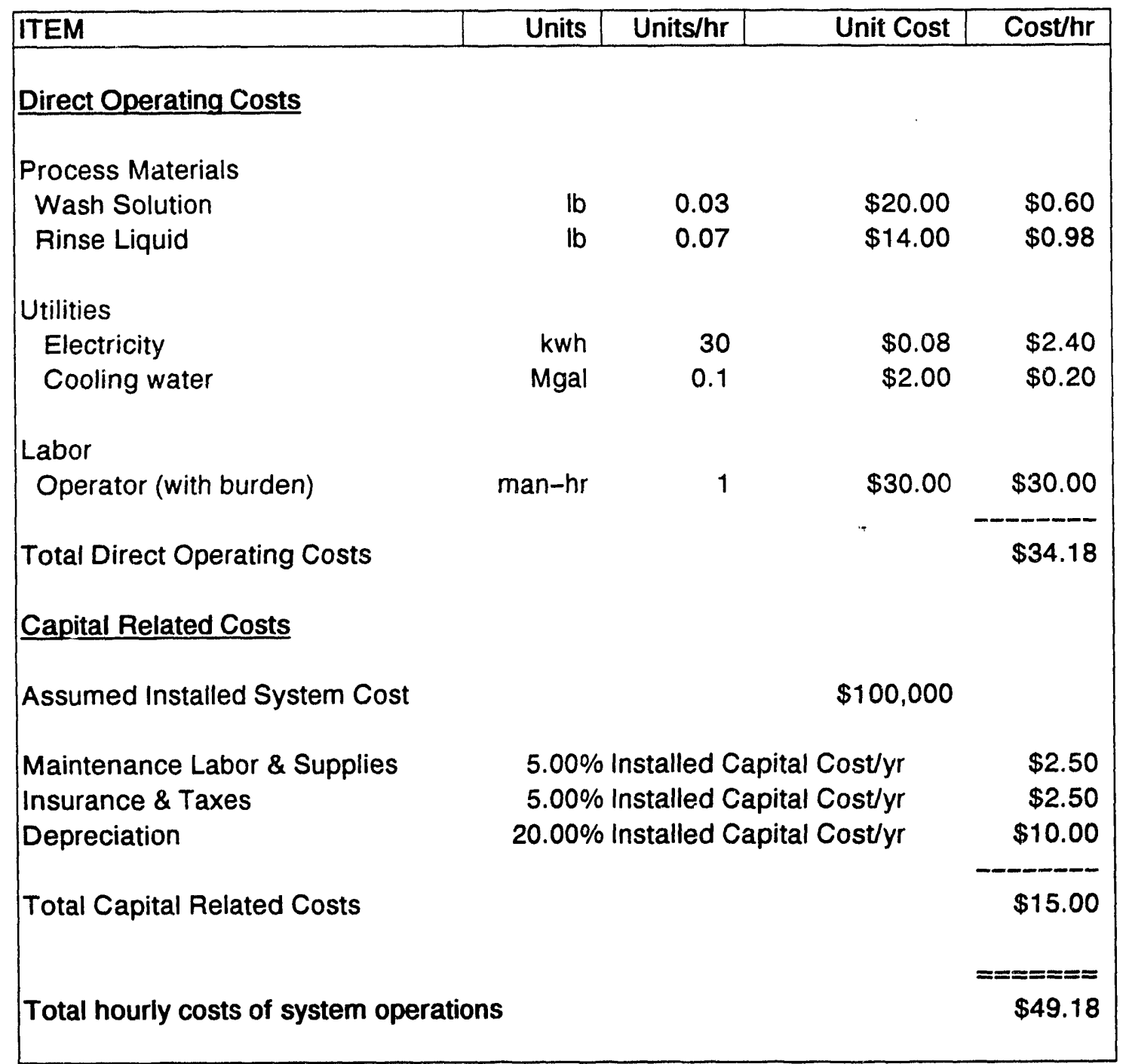


related costs are projected to be $\$ 15 /$ hour. Labor and capital costs would account for about $90 \%$ of the cost of operating the system. The cost of process materials is based on the current prices of PF 5070 and of perfluorinated surfactant and the operating losses given in Table 5.2. These losses are based on operating data provided to the $3 \mathrm{M}$ Corporation by users of the Advanced Vapor Degreasing (AVD) process which uses perfluorinated liquids as the final step of a parts cleaning process (W. Holloway, Personal Communication, March 1993).

Projected costs of system operations for a commercial scale unit are presented in Table 5.3. Direct operating costs are estimated to be of the order of $\$ 125$ per hour, based on the assumptions given in Table 5.3. Capital related costs are now projected to be the major cost element, accounting for half the cost of system operation. These costs could be reduced significantly if the unit were operated more than one shift per day. It was assumed that unit would have a dedicated operator and a half-time assistant, raising the burdened labor cost (including all indirect costs) to $\$ 45 / \mathrm{hr}$. Operating losses estimates for process liquids were provided by the $3 \mathrm{M}$ Corporation (W. Holloway, loc. cit.).

\subsubsection{Economic Incentives}

The economic incentives for operating a decontamination system are the recovery value of the equipment being processed, and the reduced cost of waste disposal, which currently runs in excess of $\$ 100 / \mathrm{ft} \wedge 3$ for low level waste $(H$. Godbee, ORNL, Oak Ridge, TN, Personal Communication, 3/93), and which is expected to increase rapidly over the next decade to possibly two to three times this value.

The effective equipment processing capacity of an ultrasonic bath is at most $20 \%$ of the bath volume. Thus, operating capacities of the prototype and full scale systems are estimated to be of the order of $0.2 \mathrm{ft}^{\wedge} 3$ and $5 \mathrm{ft}^{\wedge} 3$ of contaminated equipment per cycle. Based on the data presented in previous sections of this report, a cleaning cycle may require from $15 \mathrm{~min}$ to one hour, including rinse. It is assumed for purposes of this discussion that the average cycle time in operation will be approximately 30 minutes for these systems, i.e. two cycles/ hour. The equipment processing capabilities of the prototype and commercial systems would therefore be $0.4 \mathrm{ft}^{\wedge} 3 / \mathrm{hr}$ and $10 \mathrm{ft}^{\wedge} 3 / \mathrm{hr}$, respectively. Assuming a conservative figure of $\$ 100$ for the cost of disposing of low level radioactive wastes, and assuming further that the process results in a reduction in radioactive solid waste volume of $99 \%$ (i.e. the volume of the filter which is disposed periodically as compared to the total volume of equipm.ent processed between filter changes), and that the equipment being decontaminated has a nominal recovery value of $\$ 200 / \mathrm{ft}^{\wedge}$ (which is a conservative figure for items such as electronic instrumentation and electric motors), operating credits would be $\$ 120 / \mathrm{hr}$ and $\$ 3,000 / \mathrm{hr}$ for the prototype and commercial systems, respectively. These operating cre? its are higher than the operating costs given in Tables 5.2 and 5.3 by about $\$ 80 / \mathrm{hr}$ for the prototype system, and over $\$ 2,800 / \mathrm{hr}$ for the commercial scale system. Estimated pretax pay out times would be approximately 9 
Table 5.3 Estimated projected operating costs of a commercial scale (200 gallon bath) ESI decontamination system

\section{Operating Assumptions:}

Operating Shifts/day: 1

Operating Hours/day: 8

Operating Days/year: 200

System Life, yrs: 5

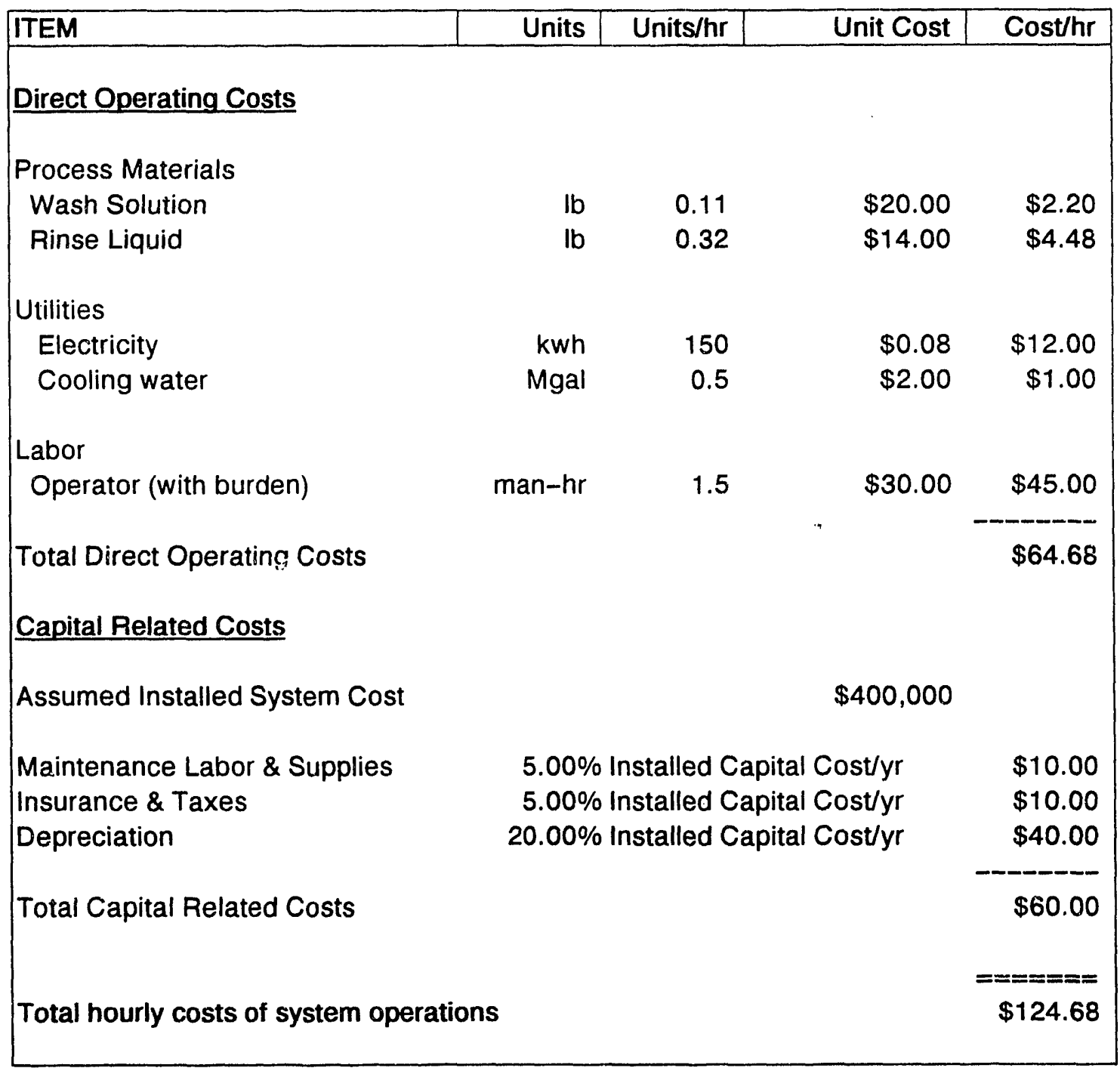


months for the prototype system and 1 month for the commercial system. Even if some of the assumptions made in the calculations are optimistic, these are extremely attractive financial figures which indicate that such a decontamination process would of high commercial interest.

\subsection{Conclusion}

A commercial scale system using the proposed ESI process is projected to be financially very attractive, and would be of high commercial interest. 


\section{FINDINGS, CONCLUSIONS, AND RECOMMENDATIONS}

\subsection{Findings}

\subsubsection{Process Liquid Stability}

While some changes in the structure of the process liquids, there was no catastrophic damage even at the highest radiation levels used (10 Mrad), and which is significantly higher than the radiation levels the process liquids would be exposed to in decontamination operations.

\subsubsection{Decontamination Studies}

The level of radioactivity of fifteen parts contaminated with micron sized, radioactive iron ( $\mathrm{Fe}(59)$ ) oxide powder was decreased by one to better than two orders of magnitude by ultrasonic cleaning in CFC-113 or perfluorinated liquids to the values indicated in the following table:

\begin{tabular}{|c|c|c|c|}
\hline $\begin{array}{c}\text { Number of } \\
\text { Samples }\end{array}$ & $\begin{array}{c}\text { Residual } \\
\text { Radioactivity } \\
\mathrm{Bq}\end{array}$ & $\begin{array}{c}\text { Residual } \\
\text { Contamination } \\
\text { decays } / \min . / 100 \mathrm{~cm}^{\wedge} 2 \\
\end{array}$ & Type of contamination \\
\hline $\begin{array}{r}15 \\
13 \\
12 \\
5\end{array}$ & $\begin{array}{l}<38 \\
<9 \\
<3 \\
<1\end{array}$ & $\begin{array}{l}<11,000 \\
<2,200 \\
<1,100 \\
<\quad 220\end{array}$ & $\begin{array}{l}\text { Fixed, Low risk } B \text { or } x \text {-ray } \\
\text { Removable, Low risk } B \text { or } x \text {-ray } \\
\text { Fixed, } B \text { or } x \text {-ray } \\
\text { Removable, } B \text { or } x \text {-ray }\end{array}$ \\
\hline
\end{tabular}

The residual contamination values cited above are the radiation limits listed in NRC Regulatory Guide 8.23 for allowable radiation limits in unrestricted areas for fixed and removable contaminants containing either low risk beta or x-ray emitters, such as the powders used in the study, or unspecified beta or x-ray emitters.

The differences in decontamination levels achieved is mainly a function of parts complexity.

Decontamination occurs rapidly, with most of the radioactive particles being removed with the first minutes of sonification.

\subsubsection{Filtration Studies}

Filtering the contaminated process liquids from the parts decontamination operation through a $0.1 \mu \mathrm{m}$ rated membrane microfilter quantitatively removes the radioactive 
iron oxide particles from suspension. Within the statistics of the measurements, the filtrates were found not to be radioactive.

\subsubsection{Projected Process Economics}

It is projected that the capital cost of a commercial scale unit capable of processing $10 \mathrm{ft}^{\wedge} 3 / \mathrm{hr}$ of radioactive waste would be approximately $\$ 400,000$. Operating costs on a one shift basis are estimated to be $\$ 125 / \mathrm{hr}$. Assuming credits, conservatively estimated to be only $\$ 300 / \mathrm{ft}^{\wedge} 3\left(\$ 100 / \mathrm{ft}^{\wedge} 3\right.$ for reduced waste disposal costs and $\$ 200 / \mathrm{ft}^{\wedge} 3$ for the value of the recovered equipment), the system would be financially attractive with a pretax pay back time of 1 month.

\subsection{Conclusions}

\subsubsection{Process Liquid Stability}

Radiation stability is not a barrier to the use of perfluorinated liquids as decontamination media.

\subsubsection{Decontamination Studies}

Sonica ion of a part contaminated with radioactive particles in a fluorinated liquid is an effective method of decontamination. Perfluorinated surfactant solutions are at least as effective as perfluorinated liquids and CFC-113 as decontamination media, and may be more effective.

\subsubsection{Filtration Studies}

The process liquids can be simply decontaminated by filtration, and can thus be recycled for further use. The filter also provides a simple, compact support for the disposal of the radioactive particles removed from the parts being decontaminated.

\subsubsection{Projected Process Eccnomics}

A commercial scale system using the proposed ESI process is projected to be financially very attractive, and would be of high commercial interest.

\subsubsection{Overall Conclusions}

The proposed ESI decontamination process is technically effective and economically attractive. 


\subsection{Recommendations}

It is recommended that the ESI decontamination process be further developed in a Phase II program. 


\section{REFERENCES}

K.C. Ashley, "Precision Cleaning Using High Pressure FreonR", Proc. 30th Annual Technical Meeting of the Institute of Environmental Sciences, p. 20 23. 1984.

J.A. Ayres, "Decontamination of Nuclear Reactors and Equipment", Chapter 1, p. 4, The Ronald Press Company, New York, NY, 1970.

R.D. Bond and A. Kearsey, "Ultrasonic and Immersion Cleaning: a Comparison using Aqueous and Fluorocarbon Solvents", Report AEEW-R-1903, AEE Winfritł (UK), November 1984.

E.I. Du Pont de Nemours and Co., Inc., "Krytox Fluorinated Oils", p. 6, Wilmington, DE 19898, Februery 1983.

P.M. Gallagher, V.J. Krukonis, and R. Kaiser, "Freon (CFC-113) Solvent Replacement: A New Process for Precision Parts Cleaning", Phase I Final Report, Prepared for USAF, Ballistic Missile Organization, Norton AFB, CA 92409-6468, Contract No. F04704-91-C-0033, Jan. 1992.

R.F. Heine, "Some Effects of Ionizing Radiation on Fluorocarbon Liquids", J. Physical Chemistry, 66, 2116-2118, 1962.

R. Kaiser, and R. Rosensweig, "Study of Ferromagnetic Liquid", NASA CR-1407, August 1969.

R. Kaiser, "Particle Dispersion Studies", AFTAC Report VT/1415, ARPA Order No. 1702, August 1973.

R. Kaiser, U.S. Patent 4,711,256, "Method and Apparatus for Removal of Small Particles from a Surface", 12/8/1387.

R. Kaiser, "Enhanced Removal of Sub-Micron Particles from Surfaces by High Molecular Weight Fluorocarbon Solutions", in "Particles on Surfaces 2:

Detection, Adhesion and Removal", K.L. Mittal, Editor, Plenum Press, New York, NY, 1989.

R. Kaisєr, U.S. Patent 3,784,471, "Solid Additives Dispersed in Perfiuorinated Liquids with Perfluoroalkyl Ether Dispersants", 1/8/1974. 
M.K.W. Ko. G. Molnar, and D. Chang, "Preliminary Report on the Direct Greenhouse Warming of CFCs Replacement Chemicals", Prepared for 3M Industrial Products Division, St. Paul, MN 55144, Atmospheric and Environmental Research, In ... Cambridge, iMA, September 1991.

E.M. Lifschitz, J. Exp. Theor. Phys. USSR, 29, 94, 1954.

F. London, Z. Physik, 63, 245, 1930.

E.L. Mackor, J. Colloid Sci., 6, 492, 1951.

W.N. Rankin and J.F. McGlynn, "Overview of Decontamination Technology", DOE Report WSRC-RP-89-1080 (also CONF-900132-2, 1990).

R.S. Stolarsky and R.D. Rundel, "Fluorine Photochemistry in the Stratosphere", Geophysical Research Letters, 2, 443-444, 1975.

D. Tabor, J. Coll. Inter. Sci., 31(3), 364, 1969.

U.S. Regulatory Commission, Office of Standard Development, Regulatory Guide 8.23, "Radiation. Safety Surveys at Medical Institutions", February 1979.

E.J.W. Verwey and J. Th. G. Overbeek, "Theory of the Stability of Lyophobic Colloids", Elsevier Publishing Co., Amsterdam, 1948.

A.D. Zimon, "Adhesion of Dust and Powder", p. 30, Plenum Press, New York, NY, 1969 

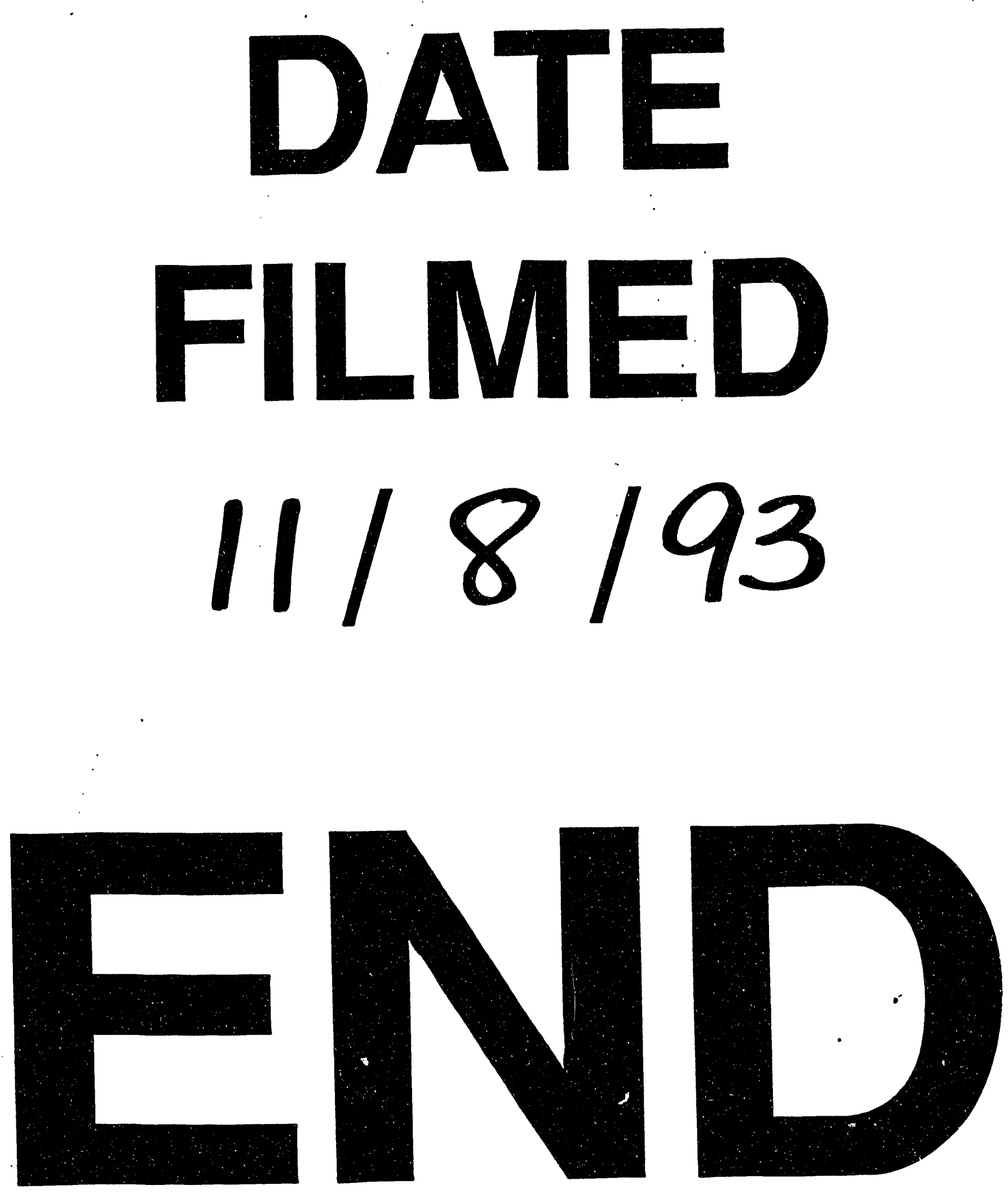
$=$
$=$ 